NBER WORKING PAPER SERIES

\title{
SOCIOECONOMIC NETWORK HETEROGENEITY AND PANDEMIC POLICY RESPONSE
}

\author{
Mohammad Akbarpour \\ Cody Cook \\ Aude Marzuoli \\ Simon Mongey \\ Abhishek Nagaraj \\ Matteo Saccarola \\ Pietro Tebaldi \\ Shoshana Vasserman \\ Hanbin Yang \\ Working Paper 27374 \\ http://www.nber.org/papers/w27374
NATIONAL BUREAU OF ECONOMIC RESEARCH
1050 Massachusetts Avenue
Cambridge, MA 02138
June 2020

We are extremely grateful to Mark Cullen and Suzanne Tamang at the Center for Population Health Sciences at Stanford University, and to Alexei Pozdnukhov at Replica for the ongoing cooperation. Alex Weinberg provided outstanding research assistance throughout this project. We also thank Stephen Eubank and the University of Virginia Biocomplexity Institute, Tim Bresnahan, Matt Jackson, and Mike Whinston for their insightful comments. The views expressed herein are those of the authors and do not necessarily reflect the views of the National Bureau of Economic Research.

At least one co-author has disclosed a financial relationship of potential relevance for this research. Further information is available online at http://www.nber.org/papers/w27374.ack

NBER working papers are circulated for discussion and comment purposes. They have not been peer-reviewed or been subject to the review by the NBER Board of Directors that accompanies official NBER publications.

(C) 2020 by Mohammad Akbarpour, Cody Cook, Aude Marzuoli, Simon Mongey, Abhishek Nagaraj, Matteo Saccarola, Pietro Tebaldi, Shoshana Vasserman, and Hanbin Yang. All rights reserved. Short sections of text, not to exceed two paragraphs, may be quoted without explicit permission provided that full credit, including (C) notice, is given to the source. 
Socioeconomic Network Heterogeneity and Pandemic Policy Response

Mohammad Akbarpour, Cody Cook, Aude Marzuoli, Simon Mongey, Abhishek Nagaraj,

Matteo Saccarola, Pietro Tebaldi, Shoshana Vasserman, and Hanbin Yang

NBER Working Paper No. 27374

June 2020

JEL No. H12,H75,I18

\section{ABSTRACT}

We develop a heterogeneous-agents network-based model to analyze alternative policies during a pandemic outbreak, accounting for health and economic trade-offs within the same empirical framework. We leverage a variety of data sources, including data on individuals' mobility and encounters across metropolitan areas, health records, and measures of the possibility to be productively working from home. This combination of data sources allows us to build a framework in which the severity of a disease outbreak varies across locations and industries, and across individuals who differ by age, occupation, and preexisting health conditions.

We use this framework to analyze the impact of different social distancing policies in the context of the COVID-19 outbreaks across US metropolitan areas. Our results highlight how outcomes vary across areas in relation to the underlying heterogeneity in population density, social network structures, population health, and employment characteristics. We find that policies by which individuals who can work from home continue to do so, or in which schools and firms alternate schedules across different groups of students and employees, can be effective in limiting the health and healthcare costs of the pandemic outbreak while also reducing employment losses.

Mohammad Akbarpour

Stanford University

Graduate School of Business

Mohamwad@stanford.edu

Cody Cook

Graduate School of Business

Stanford University

codycook@stanford.edu

Aude Marzuoli

8024 Conser Street

Overland Park, KS 66204

aude@replicahq.com

Simon Mongey

Kenneth C. Griffin Department of Economics

University of Chicago

1126 E. 59th Street

Chicago, IL 60637

and NBER

mongey@uchicago.edu

Abhishek Nagaraj

University of California, Berkeley

abhishek.nagaraj@gmail.com
Matteo Saccarola

University of Chicago

5757 S University Avenue

3rd Floor

Chicago, IL 60637

msaccarola@uchicago.edu

Pietro Tebaldi

Department of Economics

University of Chicago

5757 S. University Avenue

Chicago, IL 60637

and NBER

ptebaldi@uchicago.edu

Shoshana Vasserman

Stanford Graduate School of Business

655 Knight Way

Stanford, CA 94305

svass@stanford.edu

Hanbin Yang

Harvard University

hanbin.v.yang@gmail.com

Project Website is available at: www.reopenmappingproject.com 


\section{Introduction}

\subsection{Overview}

Our goal is to compare the effects of different policies during the COVID-19 pandemic accounting for public health and economic trade-offs within the same empirical framework. The multi-dimensional consequences of different policies depend critically on both the mobility patterns and the socioeconomic and health structures of a given geographic area. To take this into account, we develop a model in which interactions between individuals are governed by a contact network, and individuals belong to heterogeneous groups based on location, age, industry, and health status.

Using data on individual mobility based on cell-phones location data, complemented with data from the Framework for Reconstructing Epidemiological Dynamics (FRED) (Grefenstette et al. 2013), we capture patterns of movement between different groups. Frequency of close encounters and the demographics of these encounters constitute a key mechanism that underlies the spread of an infectious disease, as well as its health and healthcare consequences (death, hospitalizations, costs). Furthermore, distinct types of economic activity contribute differently to the distribution of encounters, and may be more or less replaceable with "work from home".

The model links explicitly alternative policies to their impact on the distribution of encounters across different types, as well as on the economic activities in which encounters are made. As such, it allows us to measure the health, healthcare, and economic impact of alternative policies accounting for heterogeneity across individuals in behavior, ability to fight the infection, and ability to work from home.

We focus on comparing policies that alter mobility and encounters, such as shelter-in-place orders, school closures, industry closures, rotation of workdays or work hours, and isolation of frail, high-risk individuals. Our model can also be used to study the importance of policies and behavioral changes (e.g., wearing masks) that can lower exposure and infection without reducing encounters. In terms of outcomes, for each sequence of policies, and across different locations, we measure death, hospitalizations, ICU access, and number of workers who are not productive because either sick, quarantined, and/or unable to work from home when requested to shelter-in-place.

\subsection{Data}

We combine the following primary sources, which we introduce in details in Section 3 . (i) synthetic populations at the MSA level from Replica and FRED 11 (ii) electronic medical records from the COVID-19 Research Database $2^{2}$ (iii) occupation-level data from the Occupation Information Network $\left(\mathrm{O}^{*} \mathrm{NET}\right)$, combined with the Occupation Employment Statistics (OES)

\footnotetext{
${ }^{1}$ See https://replicahq.com/ and https://fred.publichealth.pitt.edu.

${ }^{2}$ See https://covid19researchdatabase.org/.
} 
and the American Community Survey (ACS); (iv) comorbidity and demographic information from the Medical Expenditure Panel Survey (MEPS).

\subsection{Results}

Our current finding: $\mathrm{S}^{3}$ highlight differences between policies across MSAs, as driven by the composition of the local population, mobility and encounter patterns, and initial infections in the early months of 2020. A cautious reopening of all activities will lead in higher cases, with areas where the pandemic outbreak was less severe in early 2020 expected to experience a faster growth in infections, hospitalizations, ICU admissions, and deaths.

Policies that lower contact between individuals while trying to limit employment losses can be very effective. When focusing on asking individuals who are able to work from home to do so, or when alternating school and work schedules to lower density of encounters, we predict a significant reduction in cases (up to $40 \%$ fewer deaths in Chicago, and $17 \%$ fewer deaths in New York), while employment losses are contained relative to a regime in which only essential activities are open.

\subsection{Related Literature}

Our work is related to an already expansive economics literature analyzing the COVID-19 pandemic (e.g., Budish, 2020; Baker et al., 2020, Bartik et al., 2020; Mongey et al., 2020, Dingel and Neiman, 2020; Fernández-Villaverde and Jones, 2020)). In particular, we add to the burgeoning set of papers that look at the effectiveness of different policies to open the economy while the crisis is still ongoing (Acemoglu et al., 2020, Baqaee and Farhi, 2019, Benzell et al., 2020; Farboodi et al., 2020; Glover et al., 2020; Birge et al., 2020; Loertscher and Muir, 2020; Azzimonti et al., 2020). Stock (2020) provides an overview of this literature.

Much more broadly, our work adds to the vast - and daily growing - interdisciplinary research on the containment measures for the COVID-19 pandemic, building on multi-agent model-based epidemiological work leveraging data on social contacts (Eubank et al., 2004, Grefenstette et al. 2013). Related studies in this literature include Eubank et al. (2020); McCombs and Kadelka (2020); Prem et al. (2020); Moran et al. (2020); Gatto et al. (2020); Soriano-Paños et al. (2020); Will et al. (2020). New articles appear every day. We contribute to this work by adding an explicit consideration of the employment effect of different policies, also accounting for the extent to which individuals are able to work from home.

Further, our model considers explicitly the interlinked relationship between (labor) production, health, and healthcare. This follows a classical framework in health economics built upon Grossman (1972), and more recently adopted in Aizawa and Fang (2020). In modelling how unhealthy individuals need healthcare, and are not productive, we leverage a novel data source

\footnotetext{
${ }^{3}$ The reader can find updated material referring to this project at www.reopenmappingproject.com. As more data become available, we will refine and update our results.
} 
providing millions of health records for US individuals. This data is complemented with findings from early studies analyzing the properties of Sars-CoV-2, including Novel et al. (2020); Mizumoto et al. (2020); Verity et al. (2020); Ruan (2020). New papers become available every day.

\section{Model}

\subsection{Contact Network}

We are interested in the diffusion of an infectious disease in a contact network of individuals. A contact network is a simple graph, consisting of a set of individuals and set of pairs of individuals who are connected. Only connected nodes can infect each other. An individual is characterized not only by her network position, but also her age, industry, and health status.

A social planner can restrict interactions between people and thus impose a different structure on the contact network. For instance, by closing down schools, a social planner can ensure that there will be little or no connections left between students. Thus, any policy is effectively choosing a subset of connections and removing them from the possible networks. The following stylized example referring to Figure 1 clarifies our approach.

EXAMPLE 1 Consider the simple stylized network of individuals as shown in Figure 1. Different colors and shapes indicate different types of agents. The green squares are children, grouped in two schools and the red circles are adults who work in a manufacturing firm. The three black triangles are adults who work in a tech firm, and the blue diamonds do not work and are only in contact with their family members.

Figure 1 a is the network in normal (non-pandemic) times. In Figure 1b, both schools and the firm employing red-circle individuals are closed. The network has now two separate components, with one household being completely isolated in the top-left corner. Figure $1 \mathrm{c}$ is the network structure when the firm employing black-triangle individuals is also closed. The network is now divided into four small, isolated components.

Our framework allows us to consider policies such as "Individuals should work from home if possible, and schools are closed." Figure 1d shows the network structure in this situation. All individuals in the tech firm (black) can work from home. One of the workers in the manufacturing firm (red) can also work from home. The network now has more connections than a total shut down, so there is more expected infection or death. At the same time, however, our model will predict a much lower drop in productive employment.

Outcomes generated by a given policy depend crucially on the underlying network structure and the types of individuals within it. Individuals in our framework are heterogeneous in a variety of aspects; for instance, their probabilities of symptomatic infection given exposure, their probabilities of needing ICU treatment, and their probabilities of being able to work 
Figure 1: Illustrative example of contact network and social distance policies

(a) Normal configuration, two firms, two schools

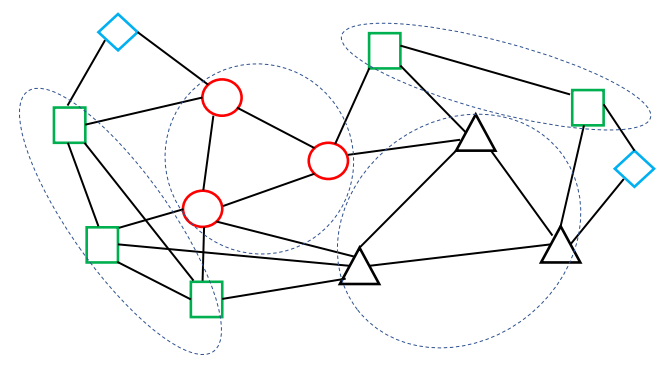

(c) All firms and schools shut down

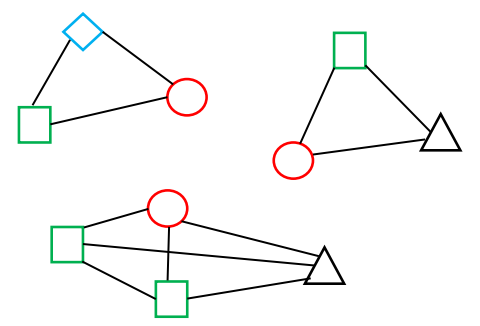

(b) Schools shut down and red firm shuts down

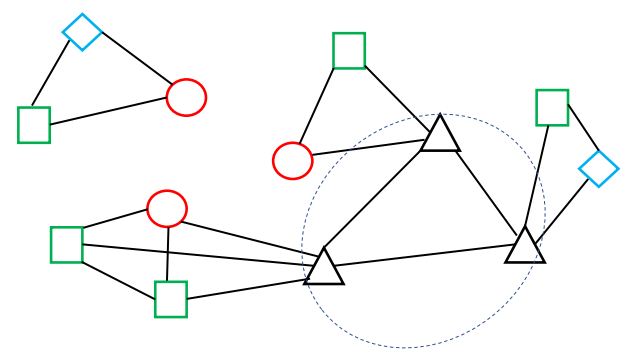

(d) Work from home when possible, no schools

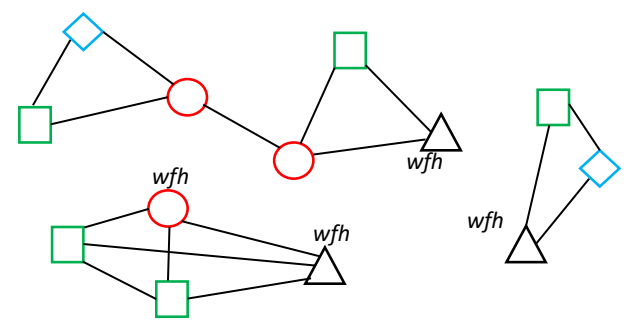

Note: The figure displays a stylized example of a network of heterogeneous individuals grouped in households, schools, and firms, under different policies, where 1 ia is the configuration during normal times. Green squares nodes correspond to children, red circles correspond to workers of a manufacturing firm, black triangles to workers of a tech firm, and blue diamonds indicate individuals who are not working. In panel[1b red firm and schools are closed, in panel $1 \mathrm{c}$ all firms and schools are closed, and in panel $1 \mathrm{~d}]$ schools are closed and those who can work from home, while others are allowed to go to work.

productively from home, and so on. Our framework allows us to study how differences in the network structure induced by different policies (such as those in the example above) lead to differences in the rate of infection, the death toll, unemployment, and healthcare spending.

\section{$2.2 \quad$ Empirical Framework}

\subsubsection{From Contact Network to Type-based Contact Matrices}

At the scale of a MSA with millions of people, it is often computationally intractable to work with the complete contact network when modeling diffusion. As such, we reduce the dimensionality of our contact networks by constructing contact matrices and estimate their parameters from the observations of individual-level contacts in the full network 4

\footnotetext{
${ }^{4}$ The idea of constructing random networks based on contact matrices is based on the inhomogeneous random graph model studied in Bollobás et al. (2007).
} 
For a given MSA $m$, an individual is described by a type $\theta \in \Theta$. A type describes demographic characteristics (e.g., age group), employment characteristics (e.g., industry and ability to work from home), and health characteristics (e.g., obesity or diabetes). In our analysis, we include approximately $|\Theta|=250$ distinct types in each MSA.

Agents of different types and in different locations have different patterns of mobility and encounters, leading to a different number of contacts in a given period $t$, measured in days. The diffusion of a virus is then moderated by the empirical contact matrix $\mathcal{C}_{m t}$. This is a square matrix with dimension $|\Theta|$, such that each entry $\mathcal{C}_{m t}\left[\theta, \theta^{\prime}\right]$ is given by the expected number of encounters that an agent of type $\theta$ has with agents of type $\theta^{\prime}$ :

$$
\mathcal{C}_{m t}\left[\theta, \theta^{\prime}\right] \equiv \mathbb{E}\left[\# \text { encounters with type } \theta^{\prime} \mid \theta\right]
$$

In principle, an encounter is defined as any interaction in which a contagious person may infect a susceptible person. For instance, behaviors cautioned against by public health officials during the COVID-19 pandemic, such as sitting within close proximity (e.g. less than 6 feet apart), or sharing a confined indoor space, constitute encounters.$^{5}$

\subsubsection{The $\Theta$-SEIIIRRD Model}

During the epidemic outbreak, an agent of any type can be in one of eight different states, each denoted by $\left.s \in\left\{S, E, I^{A}, I^{N S}, I^{H C}, R^{Q}, R^{N Q}, D\right\}\right|^{6}$ The different states correspond to the following:

$S$ : susceptible individuals, who can contract the virus if exposed;

$E$ : exposed individuals, not infectious;

$I^{A}$ : recently infected and infectious individuals;

$I^{N S}$ : infectious individuals who will not show symptoms, and will go undetected 7

$I^{H C}$ : detected and/or symptomatic infectious individuals;

$R^{Q}:$ recovered and quarantined individuals;

$R^{N Q}$ : recovered and not quarantined individuals;

$D$ : deceased individuals.

\footnotetext{
${ }^{5} \mathrm{~A}$ detailed description of how encounters are defined in our model is provided in Section 3 . The parameter regulating disease transmission within our model is calibrated with respect to this. A broader definition-which yields a higher expected number of interactions in which contagion may occur - corresponds to a lower probability of contagion upon contact.

${ }^{6}$ See also http://covid-measures . stanford.edu/for a similar model developed by Erin Mordecai and coauthors at Stanford University; our model is more general than the one used in Acemoglu et al. (2020), or similar recent and forthcoming research.

${ }^{7}$ See Berger et al. (2020) for a discussion on the modelling choices to capture both probabilistic transitions between infectious and symptomatic, and the duration within each compartment of the epidemic model.
} 
In a given period, each individual is characterized by a type-state pair $(\theta, s)$. We use the notation $S_{\theta m t}$ to indicate the share of individuals of type $\theta$ in the susceptible state $S$ in MSA $m$ in day $t, E_{\theta m t}$ to indicate exposed individuals of type $\theta$ in MSA $m$ in day $t$, and similarly for any other state.

For any type $\theta$, except for types corresponding to active healthcare workers, given the contact matrix $\mathcal{C}_{m t}$, the law-of-motion governing the transitions across the SEIIIRRD states is the following:

$$
\begin{aligned}
& \dot{S}_{\theta m t}=-\beta S_{\theta m t} \sum_{\theta^{\prime} \in \Theta} \mathcal{C}_{m t}\left[\theta, \theta^{\prime}\right] \times \underbrace{\left(I_{\theta^{\prime} m t}^{A}+I_{\theta^{\prime} m t}^{N S}\right) / N_{\theta^{\prime} m t}}_{\text {infectious not sequestered among } \theta^{\prime}} \\
& \dot{E}_{\theta m t}=+\beta S_{\theta m t} \sum_{\theta^{\prime} \in \Theta} \underbrace{\mathcal{C}_{m t}\left[\theta, \theta^{\prime}\right] \times\left(I_{\theta^{\prime} m t}^{A}+I_{\theta^{\prime} m t}^{N S}\right) / N_{\theta^{\prime} m t}}_{\text {contacts with infectious } \theta^{\prime} \text { for type } \theta}-\epsilon_{\theta} E_{\theta m t} \\
& \dot{I}_{\theta m t}^{A}=+\epsilon_{\theta} E_{\theta m t}-\tau_{\theta} I_{\theta m t}^{A} \\
& \dot{I}_{\theta m t}^{H C}=+\psi \tau_{\theta} I_{\theta m t}^{A}-\gamma_{\theta}^{H C} I_{\theta m t}^{H C}-\delta_{\theta} I_{\theta m t}^{H C} \\
& \dot{I}_{\theta m t}^{N S}=+(1-\psi) \tau_{\theta} I_{\theta m t}^{A}-\gamma^{N S} I_{\theta m t}^{N S} \\
& \dot{R}_{\theta m t}^{Q}=+\gamma_{\theta}^{H C} I_{\theta m t}^{H C}-\eta R_{\theta m t}^{Q} \\
& \dot{R}_{\theta m t}^{N Q}=+\gamma^{N S} I_{\theta m t}^{N S}+\eta R_{\theta m t}^{Q} \\
& \dot{D}_{\theta m t}=+\delta_{\theta} I_{\theta m t}^{H C} .
\end{aligned}
$$

The parameters governing these transitions between states are the following:

$\beta_{m t}$ : probability of contagion given contact between an infectious individual and a susceptible individual in MSA $m$ and day $t$;

$\epsilon_{\theta}$ : transition probability (inverse duration) from exposure to infection for type $\theta$ individuals;

$\tau_{\theta}$ : transition probability (inverse duration) from infection to either symptomatic (or detected) state or never-symptomatic and undetected state;

$\psi$ : probability that an individual is symptomatic or detected after infection;

$\gamma_{\theta}^{H C}$ : inverse duration of the infection for individuals who recover from healthcare; $\gamma^{N S}$ : inverse duration of the infection for asymptomatic, undetected individuals;

$\delta_{\theta}$ : probability of death after healthcare for infected, symptomatic type $\theta$ individuals;

$\eta$ : inverse duration of quarantine period. 
Important Note on Parameters: The parameters of our model capture a spurious combination of virological properties, behavioral factors, and modelling choices. We highlight this explicitly by letting the probability of contagion given contact vary by MSA and day. This parameter depends on a multitude of factors, which include our exact definition of contact (see discussion in Section (3), the extent to which individuals adopt protective measures (e.g., use of masks, gloves, and hand sanitizer or 6 feet distancing), local factors such as weather or genetic properties of the population. It is essential for the reader to keep this in mind when interpreting our results: policies, behavior, as well as changes in weather, can alter $\beta_{m t}$, thus affecting the evolution of the infectious disease outbreak. We aim to avoid any confusion when discussing our findings in Section 4 .

Another important parameter that can be affected by policy or behavior is $\psi$, which regulates the probability that an individual is symptomatic or detected after infection. The insurgence of symptoms can be reduced by behavioral factors such as healthy choices that boost the immune system (e.g., diet, exercise, no smoking nor alcohol). Perhaps more importantly, detection can increase following widespread testing and contact-tracing policies. Extended access to tests increases $\psi$, while also changing the selection of individuals into $I^{N S}$ and $I^{H C}$. This ultimately affects the evolution of the infectious disease and its consequences.

\subsubsection{Accounting for Healthcare: The $\Theta$-SEIIIRRDhc Model}

We make two modifications to the model to account for healthcare. First, as we will specify in Section 3 below, we do not only consider death as a relevant epidemic outcome. We also keep track of hospitalizations, and ICU usage. Different types will have different propensity of using healthcare services when infected.

Second, we note that healthcare workers are special types, since they are exposed to ill, infectious individuals. In many countries, much of the diffusion of Sars-CoV-2 took place in healthcare facilities. We therefore let $\mathcal{H C} \subset \Theta$ denote healthcare workers; equations (2) and (3) are replaced by the following:

$$
\begin{gathered}
\dot{S}_{\theta m t}=-\beta S_{\theta m t} \sum_{\theta^{\prime} \in \Theta} \mathcal{C}_{m t}\left[\theta, \theta^{\prime}\right] \frac{\left(I_{\theta^{\prime} m t}^{A}+I_{\theta^{\prime} m t}^{N S}\right)}{N_{\theta^{\prime} m t}}-\underbrace{\mathbf{1}_{\{\theta \in \mathcal{H C}\}} \beta^{H C} \frac{S_{\theta m t} I_{m t}^{H C}}{\mathcal{H C} \mathcal{C}_{m t}^{A c t i v e}}}_{\text {exposure in healthcare }} \\
\dot{E}_{\theta m t}=+\beta S_{\theta m t} \sum_{\theta^{\prime} \in \Theta} \mathcal{C}_{m t}\left[\theta, \theta^{\prime}\right] \frac{\left(I_{\theta^{\prime} m t}^{A}+I_{\theta^{\prime} m t}^{N S}\right)}{N_{\theta^{\prime} m t}}-\epsilon_{\theta} E_{\theta m t}+\mathbf{1}_{\{\theta \in \mathcal{H C}\}} \beta^{H C} \frac{S_{\theta m t} I_{m t}^{H C}}{\mathcal{H C}_{m t}^{\text {Active }}}
\end{gathered}
$$

These modified equations capture contagion between infectious individuals and healthcare workers, where $\mathcal{H C}_{m t}^{\text {Active }}$ denotes the number of active (non-infectious, alive, healthy) among $\mathcal{H C}$ in $m$ in day $t$. The probability of contagion is regulated by the parameter $\beta^{H C}<<<\beta_{m t}$, a natural assumption since healthcare workers wear Personal Protective Equipment (PPE). 


\subsection{Social Distance Policies and Outcomes}

We map a social distancing (and/or closure) policy to a sub-graph of the network of contacts between individuals, and to the corresponding modification of the contract matrix. In other words, if $\phi$ is a policy (e.g., schools are closed), we define $\mathcal{C}_{m t}^{\phi}$ to be the modified contact matrix under the set of restrictions implied by this policy (e.g., we exclude all contacts that occur at schools) 8

The set of possible social distancing policies that may have an impact on the economy and on public health can be quite large. For the sake of exposition, we focus our analysis on policies that limit the number of contacts between most types of people. Our main analysis considers policies that have been frequently proposed or carried out in US states and OECD countries during COVID-19. We will assume that specific policies regarding work and schoolrelated closures, accompanied by decreases in neighborhood interactions as well. This captures both voluntary limitations to social interactions, and policies such as park closures and limited capacity at grocery stores. (See Section 3.4 for a detailed description of the policies that we consider.)

We do not require policies to be static over the course of a disease outbreak. Rather, we consider a sequence of daily policies $\phi=\left(\phi_{1}, \phi_{2}, \ldots, \phi_{T}\right)$, where $\phi_{1}$ is the policy in day $t=1$, followed by $\phi_{2}$ in day $t=2$, and so on and so forth until $t=T$, the last day of our analysis. For example, $\phi$ could indicate $T_{1}$ days without any restrictions, followed by $T_{2}$ days of shelter-in-place with only essential industries and remote work allowed, followed by a sequence of re-opening authorizations. This has been the modal policy sequence in many countries affected by the COVID-19 epidemic outbreak.

Figure 2 summarizes our empirical approach and provides an overview of how we combine data from different sources to analyze and compare the downstream effects of various social distancing policies. The motivating idea of this approach is that policies that reduce the number of contacts between individuals may be effective in mitigating the diffusion of an infectious disease. However, the degree to which a given policy is effective depends on the particular changes that it induces on the graph of contacts, as well as the parameters that govern the transmissibility of the disease, the probability of death upon infection, etc.

Our approach captures a number of key outcomes that characterize the effectiveness of a policy. Under each policy, our $\Theta$-SEIIIRRDhc model allows us to calculate key health outcomes - such as the number of infections and deaths - as well as healthcare outcomes such as hospitalizations, healthcare spending etc. In addition, our model allows us to calculate key economic outcomes such as the number of individuals who are unable to work (either due to regulation or due to illness). Implications for downstream variables such as productivity, financial and firm outcomes etc. could also be assessed by future work.

\footnotetext{
${ }^{8}$ One limitation of this approach is that we do not capture re-configurations of activities in response to policies in this definition, although, in principle, such behavioral responses could be explicitly included in $C_{m t}^{\phi}$. For example, if all schools are closed but playgrounds are not, we could increase the number of contacts in playgrounds in a principled fashion.
} 
Figure 2: Overview of Empirical Approach

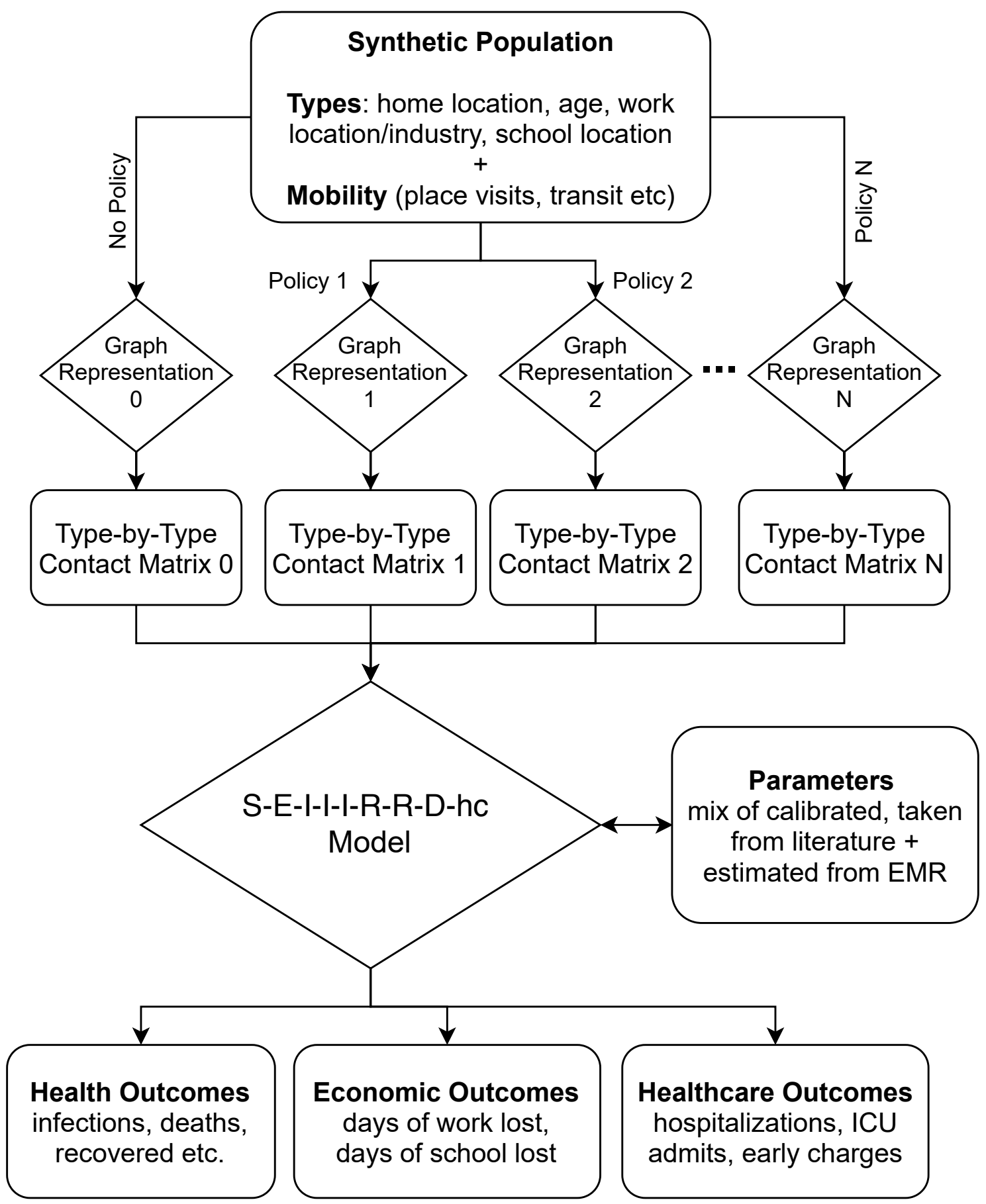

Note: This figure represents an overview of our empirical framework and how it is used to compare alternative policies along different outcomes. Note that while this figure illustrates how any given policy at a point in time $t$ (e.g., closing schools on a given day) can be studied in our model, in practice we study the aggregated effects of sequences of different policies over time (e.g., essential businesses only for 45 days followed by opening of schools). See Section 3.4 for formal details on the policy sequences we consider. 


\section{Data and Estimation}

Our empirical framework is informed by four main blocks of data: (i) data on individual characteristics and physical interactions between them to construct a contact network and contact matrices, (ii) data on health and healthcare outcomes such as diagnoses, procedures, hospitalizations, and comorbidity, (iii) data on the ability of individuals to be productive while working from home.

\subsection{Measuring Physical Contacts}

\subsubsection{Contact Network and Mobility Data}

Our primary input to construct the contact matrices that represent physical interactions between individuals of different types is synthetic population data. A synthetic population is a 1:1 scale representation of the individuals living in a given MSA, where individuals are assigned covariates in a realistic way in order to match census statistics. Importantly, the synthetic population captures information on the number of physical interactions between individuals.

Our baseline analysis for Chicago and Sacramento relies on synthetic populations and physical contacts created by processing cellphone location information from our data partner Replica .9 a startup spun-out of Alphabet Inc. Given limited coverage for the remaining MSAs, we supplement this information with data from the publicly-available FRED (Framework for Reconstructing Epidemiological Dynamics) database ${ }^{10}$ This allows us to extend our analysis to other MSAs, as we showcase in detail for New York City, one of the epicenters of the 2020 pandemic in the US.

Replica Data: Replica, has access to multiple sources of cellphone GPS data sourced from mobile applications ${ }^{11}$ as well as data on cell-tower specific locations from a major US telecom service provider. Using these inputs as well as demographic information from the Census, Replica has built a proprietary algorithm to create a synthetic population consisting of individuals who perform activities in the real world. The algorithm preserves privacy (no synthetic individual exactly matches an individual in the real world), but also produces a population that closely approximates both age and industry distributions from the Census ACS, as well as granular ground-truth data on mobility patterns from a variety of different sources (e.g., State DoT traffic counts).

For every synthetic individual, we observe a home location (and therefore the other individuals they live with). Grade school and college students are assigned a school location based on their age, home location, and the local schools. Employed residents are assigned a workplace

\footnotetext{
${ }^{9}$ Website: https://replicahq.com/; Aude Marzuoli was critical to this cooperation.

${ }^{10}$ For info visit https://fred.publichealth.pitt.edu/syn_pops.

${ }^{11}$ Unlike the more aggregate form of similar data used in the literature (e.g., Allcott et al., 2020b), these data rely on more disaggregated device-level information from multiple different sources.
} 
based on their industry of employment. Each synthetic resident is then assigned to a specific set of activities for a typical day, as predicted by the cellphone activity patterns. For each activity, we observe the time it began, the time it ended, the specific location or point-of-interest it took place at (e.g., workplace, school, parks, and grocery stores), and the mode of transport to the next activity (e.g., car, subway, and bus). Our main dataset is constructed from the first quarter (Jan-Mar) of 2019 in order to represent the full function of the economy absent lockdown measures. A detailed description of these data and the methodology for generating a synthetic city is provided in Appendix A.1.

FRED Data: Since Replica data has limited coverage at this time, we use it for our baseline analysis in Chicago and Sacramento. For the remaining MSAs for which this information is not (yet) available, we use the FRED synthetic population data provided by the Public Health Dynamics Laboratory at the University of Pittsburgh (Grefenstette et al., 2013). These populations are the key input to a widely-used open-source agent-based modeling system developed to simulate infectious disease epidemics in the epidemiological literature. Similar to Replica, FRED's synthetic population provides assignments to homes, work/school locations and neighborhoods along with demographic information such as age. However, a key difference with Replica is that FRED does not use cellphone location data to create its synthetic populations and does not provide information on mobility in the real world. See Appendix A.2 for more information.

\subsubsection{Contacts and Contact Matrices}

In defining contacts, which in our framework correspond to instances where contagion is possible, we employ the following criteria. (We take a conservatively broad stance, since our data does not allow for granularity at the level of where each person sits, and the public health community has not produced a comprehensive list of activities in which transmission may occur.)

First, an individual is connected to all others in the same household. Second, they are also connected to peers at their work and school locations. Since work and school locations can be quite large and we only want to capture direct contacts (e.g., between classmates, but not the whole school), we adjust the number of school and work contacts downward using available information on average school/work sizes and timing of contacts; details in Appendix A.1 and Appendix A.2. Finally, we also want to capture contacts between individuals that occur outside of the work, school, or home context, such as when they are performing activities like buying groceries or visiting a park. 
Figure 3: Degree Distributions Across Age/Industries by MSA

(a) Chicago: Age

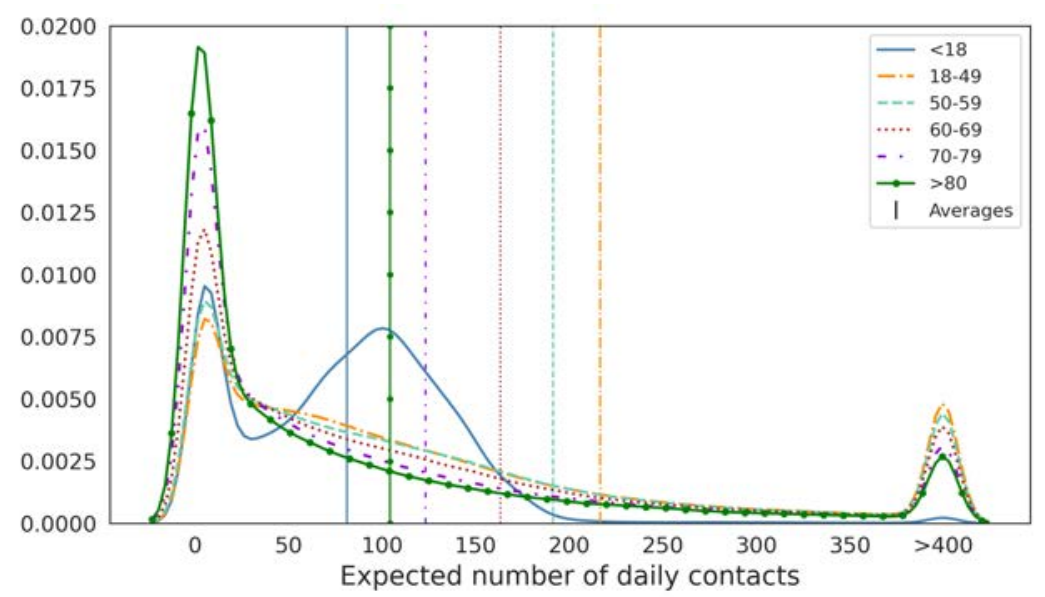

(c) Chicago: Industry

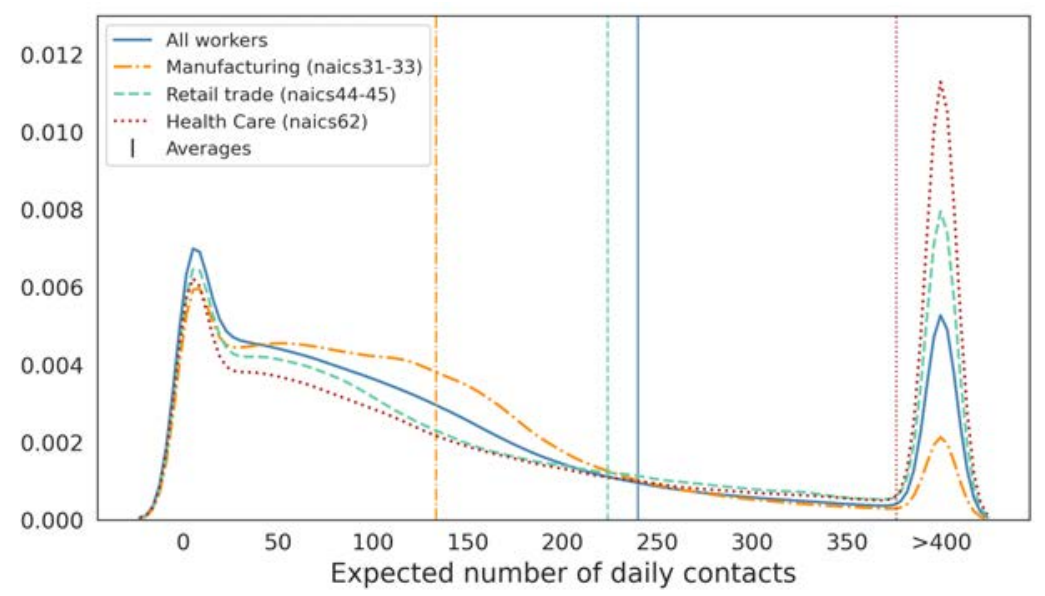

(b) Sacramento: Age

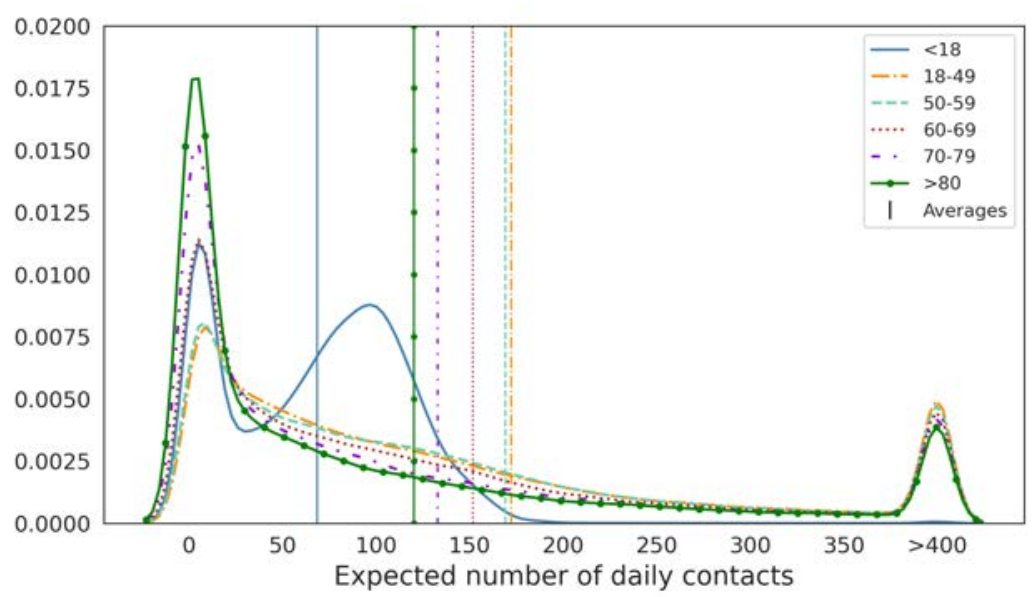

(d) Sacramento: Industry

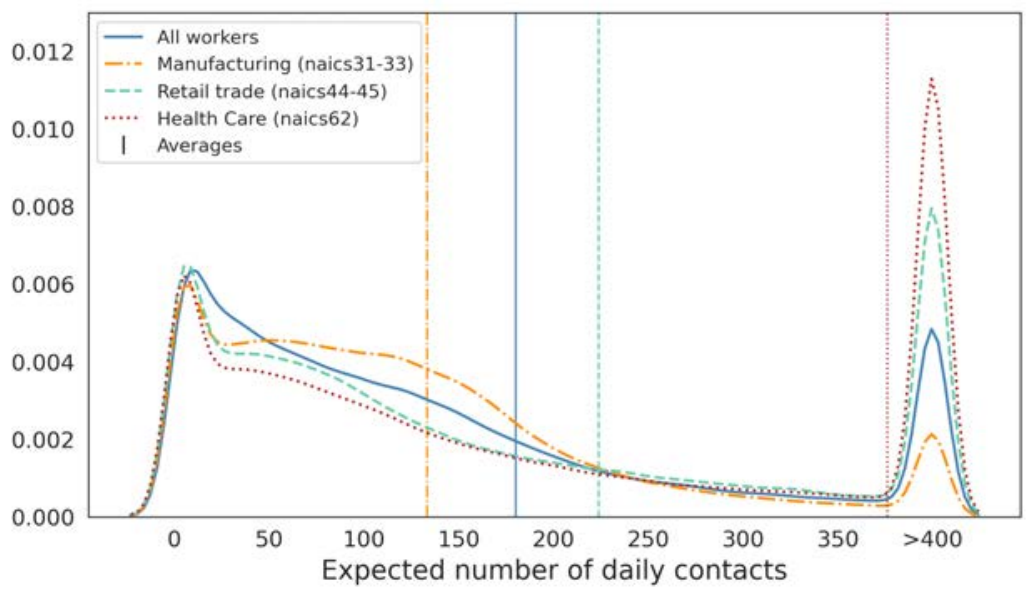

Note: This figure documents the expected number of contacts (degree) for an individual in Chicago and Sacramento as observed in the Replica synthetic populations. Contacts are split by age and industry of the focal person. The dashed vertical lines represent the average expected degree for a person of the given type. For example, the purple vertical line in panel (a) is the average degree for people 75+ years old in Chicago. Degree distributions have a long right tail, especially for workers; we cap at 500 for illustrative purposes, but the average degree is calculated on full distribution. The distribution for those under 18 is in part driven by our assumptions on class size for students; see Appendix A.1 for more details. 
While the home and school/work criteria to define a contact can be applied equally to both our data sources, the Replica data is more powerful in defining these "activity" contacts since we directly observe an individual's activities as trained on cellphone location data. In regions where Replica data is available, two (synthetic) individuals are connected when they both visit the same location and overlap in terms of the time of their visit (e.g., being inside a grocery store; at present, we count even one minute overlaps in visit time as sufficient to establish a contact). When relying on FRED data, we assume individuals are probabilistically connected to a subset of other individuals in the same neighborhood, where we define neighborhood as the same longitude and latitude rounded to the second decimal points (a squared block of slightly more than $1 \mathrm{~km}^{2}$ ).

To provide a first look of heterogeneity in individual-level contacts across MSAs, in Figure 3 we plot the frequency of expected degrees (i.e. the number of daily contacts) across individuals in a given MSA. We present these distributions for Sacramento and Chicago separated by the age and industry of a given individual, along with a vertical dotted line for the average degree for that group. The differences across age and industry, as well as across MSAs, are worth noting. In Chicago, for example, an average individual aged 25-49 encounters more than twice as many people as an individual who is 75 or older. This difference is much less pronounced in Sacramento. Across industries, healthcare has a very large number of daily encounters-more than twice as those in manufacturing.

As illustrated in Section 2, our empirical model aggregates individual contacts to a typeby-type contact matrix $\mathcal{C}_{m t}$ defined in Equation (1). To define types, we start by assigning individuals to six age bins $(0-17,18-49,50-59,60-69,70-79,80+)$ and 2-digit NAICS industry bins. Next we add two more dimensions of heterogeneity to the type space: comorbidities and the ability to work-from-home. However, while outcomes vary along these additional dimensions, contacts only depend on age and industry, and are otherwise (random and) statistically independent as long as individuals are not ill, or under social distancing policies. As more data become available, this assumption could be relaxed.

Once individuals are assigned their types, our baseline contact matrix $\mathcal{C}_{m 0}$ (i.e. absent any policy) is derived by aggregating contacts over each type-to-type combination across different activities. Formally, we let $A$ be the set of possible activities, or types of contacts: work, school, household, and neighborhood. For each type, $\xi_{\theta m}(a)$ denotes the probability that type- $\theta$ individuals engage in activity $a$ during the day, as observed in the synthetic population of MSA $m$. The data also provides us with estimates of

$$
\nu\left(\theta, a, \theta^{\prime}\right) \equiv \mathbb{E}\left[\# \text { encounters with type } \theta^{\prime} \mid \theta, \text { activity } a\right]
$$

from which we construct our initial, $t=0$, contact matrix

$$
\mathcal{C}_{m 0}\left[\theta, \theta^{\prime}\right]=\sum_{a \in A} \xi_{\theta m}(a) \nu\left(\theta, a, \theta^{\prime}\right)
$$


Figure 4: Distribution of Contacts Across Age Bins by County
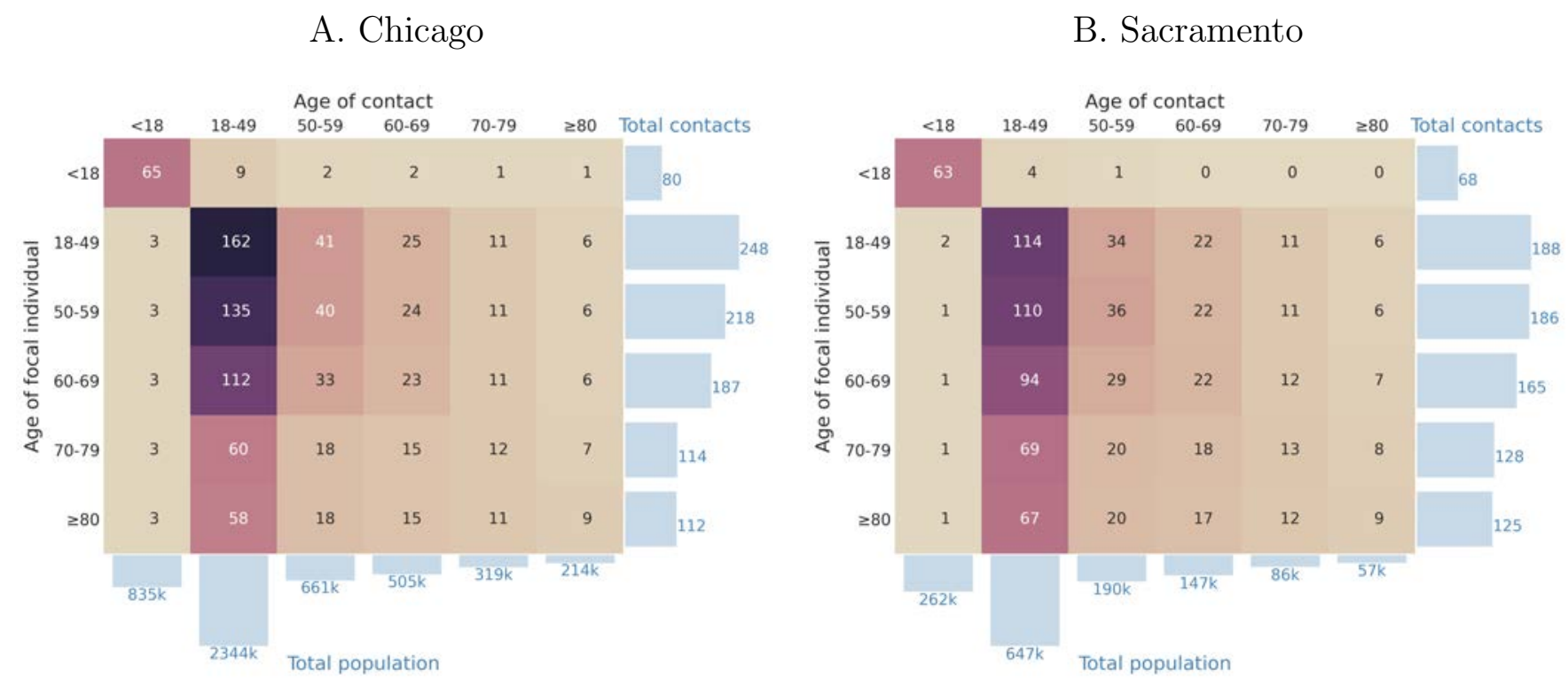

Note: Each figure represents a contact matrix for the Chicago (panel A) and Sacramento (panel B) as constructed from the Replica synthetic population. Each cell is the expected number of contacts for an individual in the row age group with individuals in the column the age group. Focal individuals must live within the specified county, but they can have contacts with those in surrounding counties. The histogram along the bottom is the population distribution across age bins. This histogram along the right is the sum of all contacts in that row.

Figure 4 provides an illustration of the resulting contact matrices across age types for Chicago (Panel A) and Sacramento (Panel B). As already highlighted in Figure 3 the Chicago MSA displays more daily encounters, particularly for the relatively young population. Nevertheless, Sacramento features more contacts between over 70 (high risk) and under 50 (lower risk) individuals. Although this figure plots the contact matrix across one dimension only (age), our model employs a multi-dimensional approach. For example, rather that simply recording contacts between 50-59 year olds and 18-49 year olds, our contact matrix records how many 50-49 year olds, who work in Manufacturing (NAICS:31-33), cannot work from home and are either obese or diabetic meet 18-49 year olds, who work in Finance and Insurance (NAICS:52), can work from home and are not obese or diabetic.

\subsection{Health and Healthcare Transitions}

\subsubsection{COVID-19 Research Database}

We work with the COVID-19 Research Database $\mathrm{L}^{12}$ (C19RD) to begin documenting the consequences of COVID-19 with detailed US healthcare data. This part of our analysis is prelimi-

\footnotetext{
${ }^{12}$ Data, technology, and services used in the generation of these research findings were generously supplied pro bono by the COVID-19 Research Database partners, acknowledged at https://covid19researchdatabase.org/.
} 
Table 1: Summary Statistics for C19RD Sample

\begin{tabular}{lccc}
\hline & Mean & St. Dev. & Obs. \\
\hline Hospitalization & 0.056 & 0.230 & 3454 \\
ICU Admission & 0.017 & 0.127 & 3454 \\
Number of Procedures & 5.561 & 6.461 & 3454 \\
Diabetic & 0.204 & 0.403 & 3454 \\
Obese & 0.339 & 0.473 & 3454 \\
Comorbidity (Diabetic or Obese) & 0.437 & 0.496 & 3454 \\
Charges & 561.7 & 1156.5 & 3454 \\
Charges if No Hospitalization & 429.8 & 547.7 & 3253 \\
Charges if Hospitalization & 2691.8 & 3704.2 & 193 \\
Charges if ICU Admission & 5167.4 & 4757.8 & 57 \\
Charges if Comorbidity & 614.9 & 1137.6 & 1508 \\
\hline
\end{tabular}

nary and subject to updates; data in the C19RD are updated weekly. Crucially, our use of the C19RD is meant to complement the daily growing literature studying the effect of Sars-CoV-2 infection and COVID-19 disease on the health and healthcare needs of different individuals. (The MIDAS network ${ }^{13}$ is an excellent source for a comprehensive overview of this literature.)

The C19RD contains regularly updated electronic medical records (EMR), and claims, for a large portion of the US population. In our current analysis, we focus on easily identifiable, diagnosed COVID-19 cases. More precisely, we apply a stringent guideline-based definition to identify a healthcare encounter related to the disease, as detailed in Appendix A.3. This leads us to a sample of 3,454 individuals living in the US with confirmed diagnoses since the onset of the epidemic disease, up to May 1st, 2020.

For each individual, we keep track of all medical procedures executed for the event of care, including indicators for hospitalization and ICU care. In terms of observables, in this timely, yet preliminary use of the data we keep track of age, diabetes, and obesity. For each case, we observe total procedure charges. This number is preliminary, since charges in EMR do not necessarily correspond to charges in the submitted claim. While reported in our summary, we currently do not use this variable in our analysis, leaving this to later updates.

The C19RD sample is summarized in Table 1. In alignment with mounting evidence from other sources (see Section 1.4), 5.6\% of cases in our sample requires hospitalization, while the ICU admission rate is $1.7 \%$. Comorbidities - currently we keep track of diabetes and obesity are observed in $43.7 \%$ of the individuals in the sample. Preliminary measures of charges are, on average, equal to $\$ 562$ per-case, but they are five times as large for hospitalized patients, and ten times as large, on average, for patients requiring ICU admission.

In Figure 5 we plot hospitalizations and ICU admission rates across age types, distinguish-

\footnotetext{
${ }^{13}$ Website: https://midasnetwork.us/covid-19/
} 
Figure 5: Frequency of Hospitalization and ICU Admissions in C19RD Sample

(a) Hospitalization Rates

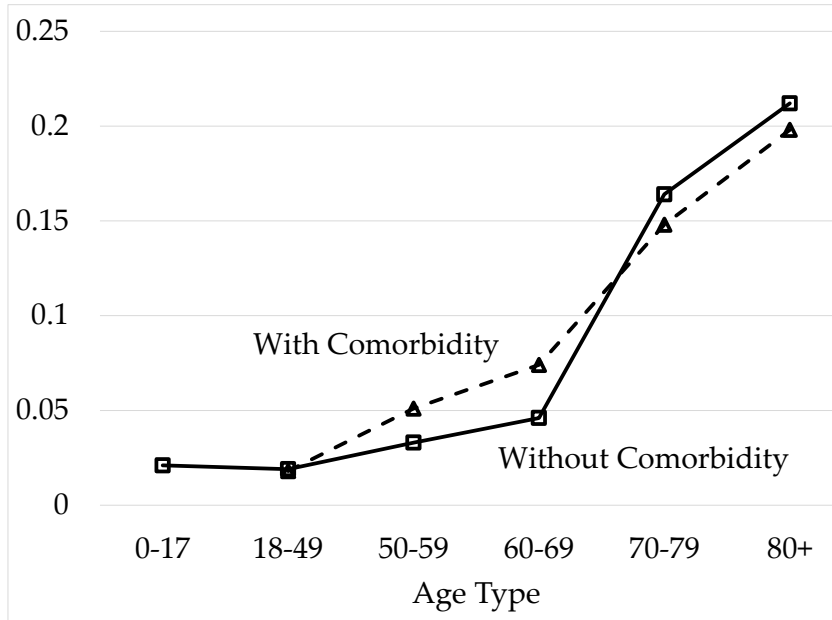

(b) ICU Admission Rates

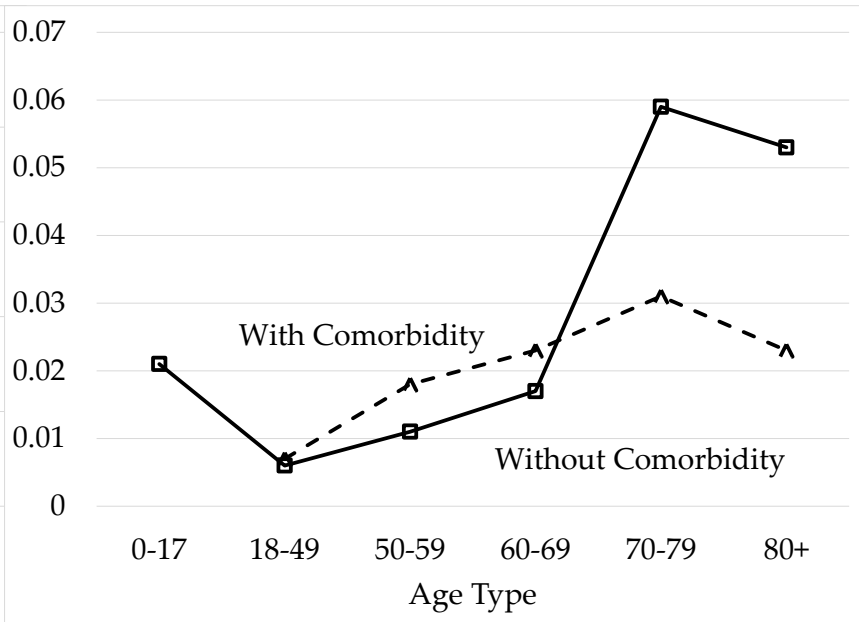

The figure shows the average hospitalization rates (left panel) and ICU admission rates (right panel) as observed in the COVID-19 Research Database sample. Each line distinguishes between individuals with and without comorbidity, while the horizontal axis distinguishes between age groups that are one dimension of a type in our empirical model. These incidences of hospitalization and ICU admissions are used to calculate hospital days and ICU days given the size of the symptomatic population $\left(I^{H C}\right)$ in our results in Section 4.

ing between cases with and without pre-exsiting comorbidities (diabetes or obesity). As known, severity increases sharply with age, showing a critical inflection point after 60 . After the age of 70, patients with comorbidities are both less likely to be hospitalized and less likely to be admitted to the ICU. This is consistent with the severity of COVID-19 being very high for these groups, implying both a higher case-fatality rate, and the difficult but rational decision of hospital staff to focus resources on patients with higher probability of survival.

\subsubsection{Comorbidity in the Medical Expenditure Panel Survey}

We enrich our definition of a type allowing each age-industry type observed in the contact matrix within a given MSA to be split in two subgroups (or sub-types): one with comorbidities, and one without comorbidities.

At the moment, we define an individual as having comorbidities if they are either diabetic or obese. The Medical Expenditure Panel Survey (MEPS), contains information on these variables, as well as individual's age, industry, and a geographic region that is a larger area covering a set of MSAs.

For any age-industry in MSA $m$, we assign a fraction $\kappa_{m}$ (age,industry) of individuals to the age-industry-MSA type with comorbidities, where

$$
\kappa_{m}(\text { age,industry }) \equiv \operatorname{Pr}[\text { diabetic or obese|age, industry, MEPS region containing } m \text { ] }
$$


Table 2: Parameters of $\Theta$-SEIIIRDhc Model

\begin{tabular}{|c|c|c|c|c|c|c|c|c|c|}
\hline \multirow[b]{2}{*}{ Age } & \multirow[b]{2}{*}{ Comorbidity } & \multicolumn{7}{|c|}{$\Theta$-SEIIIRRDhc Parameters } & \multirow{2}{*}{$\begin{array}{c}\text { Implied } \\
\text { CFR }\end{array}$} \\
\hline & & $\epsilon_{\theta}$ & $\tau_{\theta}$ & $\psi$ & $\gamma^{N S}$ & $\gamma_{\theta}^{H C}$ & $\delta_{\theta}$ & $\eta$ & \\
\hline $0-4$ & $\mathrm{~N}$ & 0.25 & 0.2 & 0.7 & 0.045 & 0.050 & 0.00000 & 0.0714 & 0.0001 \\
\hline $0-4$ & Y & 0.25 & 0.2 & $"$ & $"$ & 0.050 & 0.00001 & $"$ & 0.00015 \\
\hline $5-17$ & $\mathrm{~N}$ & 0.25 & 0.2 & $"$ & $"$ & 0.050 & 0.00001 & $"$ & 0.0001 \\
\hline $5-17$ & Y & 0.25 & 0.2 & $"$ & $"$ & 0.050 & 0.00001 & $"$ & 0.00015 \\
\hline $18-49$ & $\mathrm{~N}$ & 0.25 & 0.2 & $"$ & $"$ & 0.050 & 0.00007 & $"$ & 0.001 \\
\hline $18-49$ & Y & 0.25 & 0.2 & $"$ & $"$ & 0.050 & 0.00014 & $"$ & 0.002 \\
\hline 50-59 & $\mathrm{N}$ & 0.25 & 0.2 & $"$ & $"$ & 0.050 & 0.00036 & $"$ & 0.005 \\
\hline 50-59 & Y & 0.33 & 0.25 & $"$ & $"$ & 0.049 & 0.00071 & $"$ & 0.01 \\
\hline 60-69 & $\mathrm{N}$ & 0.33 & 0.25 & $"$ & $"$ & 0.048 & 0.00179 & $"$ & 0.025 \\
\hline 60-69 & Y & 0.35 & 0.33 & $"$ & $"$ & 0.047 & 0.00286 & $"$ & 0.04 \\
\hline $70-80$ & $\mathrm{~N}$ & 0.35 & 0.33 & $"$ & $"$ & 0.048 & 0.00214 & $"$ & 0.03 \\
\hline $70-80$ & Y & 0.35 & 0.33 & $"$ & $"$ & 0.046 & 0.00357 & $"$ & 0.05 \\
\hline $80+$ & $\mathrm{N}$ & 0.35 & 0.33 & $"$ & $"$ & 0.046 & 0.00429 & $"$ & 0.06 \\
\hline $80+$ & Y & 0.35 & 0.33 & $"$ & $"$ & 0.045 & 0.00500 & $"$ & 0.07 \\
\hline
\end{tabular}

The table lists the parameters of the $\Theta$-SEIIIRRDhc model that we use for our simulations. These parameters are based on existing literature (c.f. Section 1.4), and are subject to change. The last column on the right indicates the case-fatality-ratio for each group that is implied by our model parameters. This reflects closely results in Fletcher et al. (2020) and Ruan (2020).

is computed directly from the MEPS. The remaining fraction of individuals (with weight $1-\kappa_{m}$ (age,industry)) is assigned to the type with the same age-industry, but no comorbidities.

Importantly, contacts and comorbidities are independent, conditional on age-industryMSA; our data contains no information on differential mobility and encounters by comorbidity status. Richer data would be needed to relax this assumption.

\subsubsection{Healthcare Transitions and Model Parameters}

We use our estimates from the C19RD to study the healthcare outcomes of COVID-19 infection beside mortality. As a key ingredient for our simulations, however, the model presented in Section 2.2 .2 requires us to specify a set of parameters determining the transition across the health states of the population during the epidemic. We let some of these parameters vary by age and comorbidity.

We currently select parameters from the literature; the reader can refer to the articles cited in Section 1.4 and to the daily updates to the meta-analysis in the MIDAS network. We are extremely grateful to Mark Cullen at the Center for Population Health Sciences at Stanford University for the ongoing cooperation in tracking "sensible" parameters from the literature to use as inputs in our simulations.

The parameters we use in our $\Theta$-SEIIIRRhc model are listed in Table 2 , The only exception is $\beta_{m t}$, which we estimate by indirect inference minimizing the discrepancy between 
our predicted deaths and observed mortality series. The details on this procedure and some comments on model fit are reported in Appendix B.

\subsection{Measures of Work-from-Home and Employment}

After defining types by age, industry, and comorbidity, we add a last dimension of heterogeneity by accounting for the ability of individuals to work from home. As with comorbidity, the work-from-home dimension of a type is probabilistic: we split each age-industry-comorbidity type into two, corresponding to those able or unable to work from home, respectively. The fraction of individuals who can work from home given age and industry is $\omega$ (age, industry). Comorbidities and ability to work from home are statistically independent, an assumption which could be relaxed with richer data.

Our estimates of $\omega$ (age, industry) are derived following closely the approached introduced in Dingel and Neiman (2020) and Mongey et al. (2020). This approach combines data on activities and employment by occupation (from Occupation Information Network, O* NET, and the Occupation Employment Statistics, OES), with the composition of occupations by industry and age from the American Community Survey (ACS).

First, we use $\mathrm{O}^{*} \mathrm{NET}$ to construct a measure of the likelihood that a given 2-digit occupation $o$ may be conducted from home. For this, the $\mathrm{O}^{*} \mathrm{NET}$ contains average responses to survey questions regarding more than 250 job attributes, and is at the 6-digit occupation level. For example for the attribute, "Wear protective or safety equipment" we observe the average of worker responses that themselves range from 1 (never), to 5 (every day). We focus on 17 job attributes that reflect a job that would be difficult to reallocate to home. (See Mongey et al. 2020 , for more details.)

We then use the distribution of employment within 2-digit occupations, across 6-digit occupations from the OES to aggregate each of these scores to the 2-digit occupation level. We then assign a 2-digit occupation $o$ a work-from-home status of 1 if any of 17 job attributes have an average score of more than 3.5, indicating an average response of, for example, wearing protective equipment regularly. At the two digit occupation level this gives an indicator $w_{o} \in\{0,1\}$.

Lastly, we use national ACS data to construct the distribution of 2-digit occupations $o$ conditional on age and 2-digit NAICS: $\operatorname{Pr}(o \mid$ age, industry $) \in[0,1]$. The use of national data is motivated by the fact that MSA-level data including occupation-industry-age is not sufficiently fine for this exercise. With these we derive our desired fraction of individuals within a ageindustry who can work from home:

$$
\omega(\text { age }, \text { industry })=\sum_{o} w_{o} \times \operatorname{Pr}(o \mid \text { age, industry })
$$

This will affect both, our employment outcomes (even when the workplace is closed, the individual can work) and our policies, insofar firms and local governments can ask individuals 
to work from home if possible.

\subsection{Policies and Counterfactuals}

\subsubsection{Policy Sequences}

We use the model to collect series of health, economic, and healthcare outcomes along different sequences of policies $\phi$. We distinguish between three phases, with $t=0, \ldots, T_{1}$ indicating phase $1, t=T_{1}, \ldots, T_{2}$ for phase 2 , and $t=T_{2}, \ldots, T$ for phase 3 . These are tailored to the COVID-19 pandemic policy response across the vast majority of the United States.

We set $t=0$ corresponding to March 5th, 2020. In Phase 1, we impose no policy between March 5th and $t=T_{1}=15$, where $T_{1}=15$ corresponds to March 20th:

NP - No Policy: In the no policy regime, for $t=0,1, \ldots, T_{1}, \phi_{t}=\phi^{\mathrm{NP}}$, and the contagion patterns are determined by the contact matrix $\mathcal{C}_{m 0}$ as defined in Equation (15) above. Precisely:

$$
\begin{aligned}
\mathcal{C}_{m t}^{\phi^{\mathrm{NP}}}\left[\theta, \theta^{\prime}\right]=\mathcal{C}_{m 0}\left[\theta, \theta^{\prime}\right] & =\xi_{\theta m}(\text { household }) \nu\left(\theta, \text { household }, \theta^{\prime}\right) \\
& +\xi_{\theta m}(\text { neighborhood }) \nu\left(\theta, \text { neighborhood, } \theta^{\prime}\right) \\
& +\xi_{\theta m}(\text { work }) \nu\left(\theta, \text { work }, \theta^{\prime}\right) \\
& +\xi_{\theta m}(\text { school }) \nu\left(\theta, \text { school }, \theta^{\prime}\right) .
\end{aligned}
$$

In our baseline Phase 2, i.e. between $t=T_{1}$ and $t=T_{2}=75$, corresponding to May 19th, each MSA sets $\phi_{t}=\phi^{\mathrm{EO}}$, indicating a regime in which only essential activities are allowed. Precisely,

EO - Essential Only: Essential workplaces such as hospitals, groceries, and transportation are open, 14 all other workplaces and locations are closed. No contacts outside these locations are kept aside for all household interaction and $10 \%$ of neighborhood interaction. Formally, $\phi^{\mathrm{EO}}$ imposes

$$
\begin{aligned}
\mathcal{C}_{m t}^{\phi^{\mathrm{EO}}}\left[\theta, \theta^{\prime}\right] & =\xi_{\theta m}(\text { household }) \nu\left(\theta, \text { household }, \theta^{\prime}\right) \\
& +0.1 \xi_{\theta m}(\text { neighborhood }) \nu\left(\theta, \text { neighborhood, } \theta^{\prime}\right) \\
& +\mathbf{1}\left\{\theta, \theta^{\prime} \text { are essential workers }\right\} \xi_{\theta m}(\text { work }) \nu\left(\theta, \text { work, } \theta^{\prime}\right)
\end{aligned}
$$

In Phase 3, i.e. after May 19th we consider alternative policies between $t=T_{2}$ and $t=T=150$, corresponding to August 2nd, the last day in our analysis at the moment. During this period, $\phi_{t}$ can be one of the following:

\footnotetext{
${ }^{14}$ Essential industries corresponds to agriculture, forestry, fishing and hunting, mining, utilities, manufacturing, wholesale trade, transportation and warehousing, health care and social assistance, public administration.
} 
CR - Cautious Reopening: With $\phi_{t}=\phi^{\mathrm{CR}}$ indicating that there is no regulatory restriction on mobility or encounters; schools and workplaces are open. However, neighborhood interactions are still reduced to $10 \%$ of the normal, pre-pandemic level. This captures recommendations to use caution, limited capacity of grocery stores, and additional social distance measure enacted voluntarily. Formally, $\phi^{\mathrm{CR}}$ is such that

$$
\begin{aligned}
\mathcal{C}_{m t}^{\phi^{\mathrm{CR}}}\left[\theta, \theta^{\prime}\right] & =\xi_{\theta m}(\text { household }) \nu\left(\theta, \text { household }, \theta^{\prime}\right) \\
& +0.1 \xi_{\theta m}(\text { neighborhood }) \nu\left(\theta, \text { neighborhood }, \theta^{\prime}\right) \\
& +\xi_{\theta m}(\text { work }) \nu\left(\theta, \text { work }, \theta^{\prime}\right) \\
& +\xi_{\theta m}(\text { school }) \nu\left(\theta, \text { school }, \theta^{\prime}\right)
\end{aligned}
$$

$60+$ - Isolate 60+: With $\phi_{t}=\phi^{60+}$ indicating that there is no regulatory restriction for anyone, but individuals who are 60 or older must limit their contacts to household and neighbor (local stores). Neighborhood interactions are still reduced to $10 \%$ of the normal, pre-pandemic level. Formally, $\phi^{60+}$ is such that

$$
\begin{aligned}
\mathcal{C}_{m t}^{\phi^{60+}}\left[\theta, \theta^{\prime}\right] & =\xi_{\theta m}(\text { household }) \nu\left(\theta, \text { household, } \theta^{\prime}\right) \\
& +0.1 \xi_{\theta m}(\text { neighborhood }) \nu\left(\theta, \text { neighborhood, } \theta^{\prime}\right) \\
& +\mathbf{1}\left\{\theta, \theta^{\prime} \text { both under } 60, \text { or both essential workers }\right\} \xi_{\theta m}(\text { work }) \nu\left(\theta, \text { work, } \theta^{\prime}\right) \\
& +\xi_{\theta m}(\text { school }) \nu\left(\theta, \text { school, } \theta^{\prime}\right) .
\end{aligned}
$$

WFH - Work-from-Home if Possible: Individuals who are able to work from home productively do so, and do not form contacts at their workplace. All other employed, healthy individuals access their workplace and form contacts accordingly. Schools are open, household contacts are as usual, and neighborhood interactions limited to $10 \%$ of prepandemic levels. Formally, $\phi^{\mathrm{WFH}}$ is such that

$$
\begin{aligned}
\mathcal{C}_{m t}^{\phi^{\mathrm{WFH}}\left[\theta, \theta^{\prime}\right]} & =\xi_{\theta m}(\text { household }) \nu\left(\theta, \text { household }, \theta^{\prime}\right) \\
& +0.1 \xi_{\theta m}(\text { neighborhood }) \nu\left(\theta, \text { neighborhood, } \theta^{\prime}\right) \\
& +\mathbf{1}\left\{\theta, \theta^{\prime} \text { both cannot WFH, or both essential }\right\} \xi_{\theta m}(\text { work }) \nu\left(\theta, \text { work, } \theta^{\prime}\right) \\
& +\xi_{\theta m}(\text { school }) \nu\left(\theta, \text { school }, \theta^{\prime}\right) .
\end{aligned}
$$

AS - Alternating Schedules: Students and workers in all schools and workplaces are split into two groups that do not intersect. The thought experiment corresponds to alternating schedules (morning and afternoon), or alternating days (MWF and TThS); alternative versions could consider alternate weeks, implying different disease dynamics ${ }^{15}$ Neighborhood interactions are again reduced to $10 \%$ of pre-pandemic levels. Formally, for each

\footnotetext{
${ }^{15}$ Delays in the manifestation of symptoms and test results are key drivers of these differences. Exploring optimal alternation could be an interesting direction for future work.
} 
Figure 6: Contact Matrices Along the NP-EO-WFH Policy Sequence in Chicago

(a) $\mathcal{C}_{m}^{\phi^{N P}}$ in Chicago

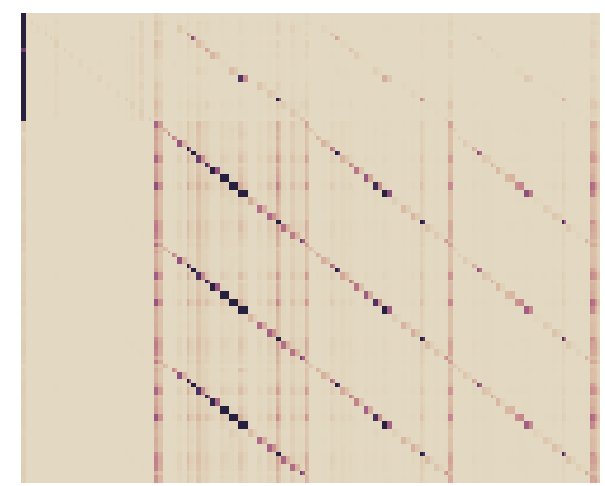

(b) $\mathcal{C}_{m}^{\phi^{E O}}$ in Chicago

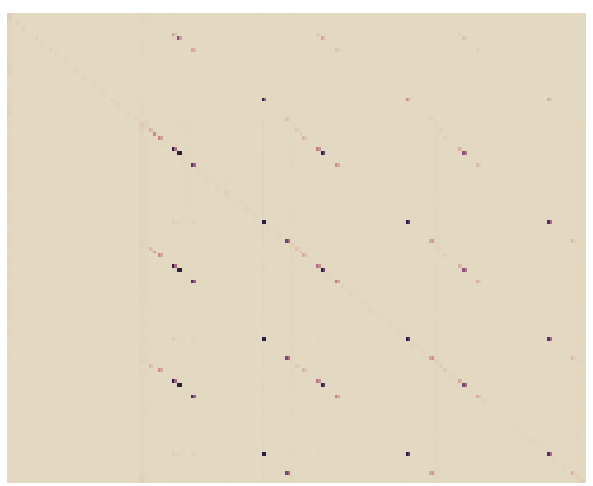

(c) $\mathcal{C}_{m}^{\phi^{W F H}}$ in Chicago

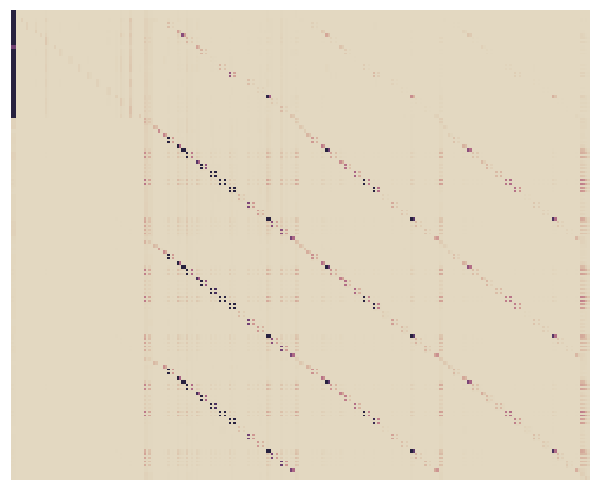

Note: The figure displays the sequence of contact matrices to study the policy sequence no policy (NP) in Phase 1, essential only (EO) in Phase 2, and work from home (WFH) if possible in Phase 3. Our simulation procedures imposes the sequence of contact matrices, and records the outcomes of the $\Theta$-SEIIIRDhc model, including active, employed individuals, and healthcare utilization.

type we consider a 50-50 split from $\theta$ to $\theta_{[1]}$ and $\theta_{[2]}$; each corresponds to one subgroup, and we suppress any workplace or school interaction between $\theta_{[1]}$ and $\theta_{[2]}$ as long as $\theta$ is non essential. ${ }^{16}$ We then have $\phi^{\mathrm{AS}}$ being such that

$$
\begin{aligned}
\mathcal{C}_{m t}^{\phi^{\mathrm{AS}}\left[\theta_{[k]}, \theta_{[\ell]}^{\prime}\right]} & =\xi_{\theta_{[k]} m}(\text { household }) \nu\left(\theta_{[k]}, \text { household, } \theta_{[\ell]}^{\prime}\right) \\
& +0.1 \xi_{\theta_{[k]} m}(\text { neighborhood }) \nu\left(\theta_{[k]}, \text { neighborhood, } \theta_{[\ell]}^{\prime}\right) \\
& +\mathbf{1}\left\{\theta_{[k]}, \theta_{[\ell]}^{\prime} \text { both essential }\right\} \xi_{\theta_{[k]} m}(\text { work }) \nu\left(\theta_{[k]}, \text { work, } \theta_{[\ell]}^{\prime}\right) \\
& +\mathbf{1}\left\{\theta_{[k]} \text { or } \theta_{[\ell]}^{\prime} \text { not essential, and } k=\ell\right\} 0.5 \xi_{\theta_{[k]} m}(\text { work }) \nu\left(\theta_{[k]}, \text { work, } \theta_{[\ell]}^{\prime}\right) \\
& +\mathbf{1}\{k=\ell\} 0.5 \xi_{\theta_{[k]} m}(\text { school }) \nu\left(\theta_{[k]}, \text { school, } \theta_{[\ell]}^{\prime}\right) .
\end{aligned}
$$

Although we limit our attentions to sequences of NP, EO, CR, 60+, WFH, and AS, our model is suited to analyze a very large class of policies. Importantly, the richer the data on activities, mobility, and encounters, the larger the set of policies one could consider.

\subsubsection{Simulations and Outcomes}

We simulate outcomes given parameters for Phase 1, and then consider combinations of EO, CR, 60+, WFH, and AS for Phase 2 and Phase 3. When discussing our results in Section 4 we will focus on sequences imposing EO in Phase 2, the modal path of most MSA in the US.

\footnotetext{
${ }^{16}$ Technically, our contact matrix now has higher dimensionality, as for each type corresponding to a nonessential worker we have two sub-types.
} 
The changes to policy in Phase 2 are retrospective, and have therefore more limited normative implications. In our current analysis we do not consider changes to Phase 1 policies, although such an analysis is feasible in our framework.

For every MSA, we start by constructing the contact matrices corresponding to the desired policy sequence $\phi$. Figure 6 shows the three matrices $\mathcal{C}_{m}^{\phi^{\mathrm{NP}}}, \mathcal{C}_{m}^{\phi^{\mathrm{EO}}}, \mathcal{C}_{m}^{\phi^{\mathrm{CR}}}$ for the Chicago MSA. This is the input to analyze the sequence NP in Phase 1, EO in Phase 2, and CR in Phase 3.

Armed with the contact matrices, we run our $\Theta$-SEIIIRRDhc model. We keep track of the number of individuals in each state (i.e. susceptible, infected etc) which allows us to calculate the total number of deaths at the end of Phase 3, a key outcome in our analysis. Beyond this outcome, we compute the number hours, individuals are unable to work under a policy sequence. This depends on the policy $\phi$, and on the epidemic outcomes, since individuals who are symptomatic do not work, and the same applies to quarantined individuals who cannot work from home. Lastly, we use our estimates of the rates of hospitalizations and ICU utilization by type, to compute the healthcare outcomes beyond death of a given trajectory of the epidemic.

\section{Results}

\subsection{Baseline Model Prediction}

We start by examining the evolution of outcomes in one MSA under the scenario in which it adopts the Cautious Reopening (CR) policy in Phase 3, following no policy (NP) in Phase 1 and essential only (EO) in Phase 2. We consider this our baseline policy sequence for two reasons. First, the sequence of NP and EO is representative of the policies that were adopted in most localities during Phases 1 and 2. Second, CR represents a policy where no direct restrictions are imposed, but which still captures a reduction of contacts in an individual's neighborhood due to social restrictions and social distancing measures ${ }^{17}$

Figure 7 plots predicted outcomes for Chicago in Phases 1, 2 and 3. In terms of epidemic outcomes, depicted in Figure 7a, the Chicago MSA experiences a growth of cases during Phase 2 - despite the EO policy - with a "peak of the curve" at $t=50$ when approximately $2.5 \%$ of the population is infected and symptomatic.

When the MSA moves from EO to CR, our model predicts that within ten days, infection rates will increase again. The new peak, at $t=115$, corresponds to almost $4 \%$ of the population being infected and symptomatic (or detected). This is reflected in a higher growth rate of cumulative deaths, and a larger number of individuals who, on a given day, need to be hospitalized or admitted to the ICU (Figure 7c). Importantly, this version of our model does

\footnotetext{
${ }^{17}$ As Allcott et al. (2020a) describe, voluntary social distancing has been broadly adopted in areas without enforceable lock-downs across the United States, and so this is likely the most representative description of how removing strong social distancing policies would manifest. (See also Brzezinski et al. (2020); Chetty et al. (2020); Gupta et al. (2020); Villas-Boas et al. (2020).)
} 
Figure 7: No Policy-Essential Only-Cautious Reopening Policy Sequence

(a) Chicago, Epidemic Outcomes

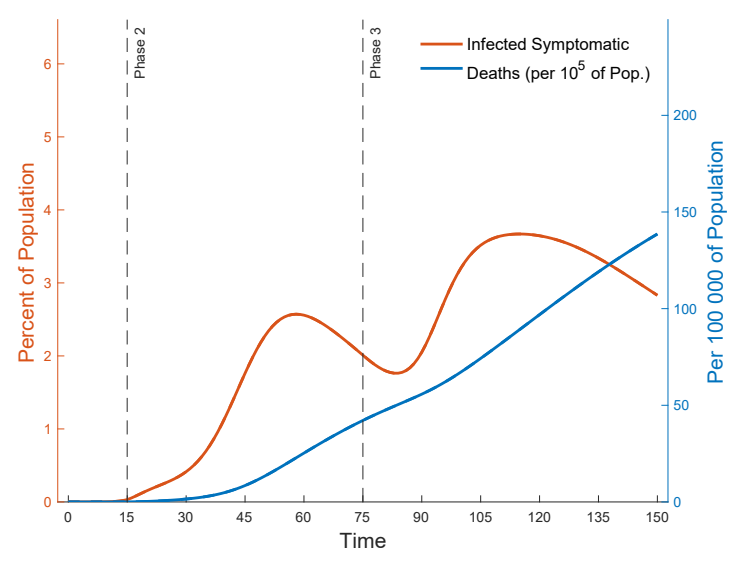

(b) Chicago, Employment Outcomes

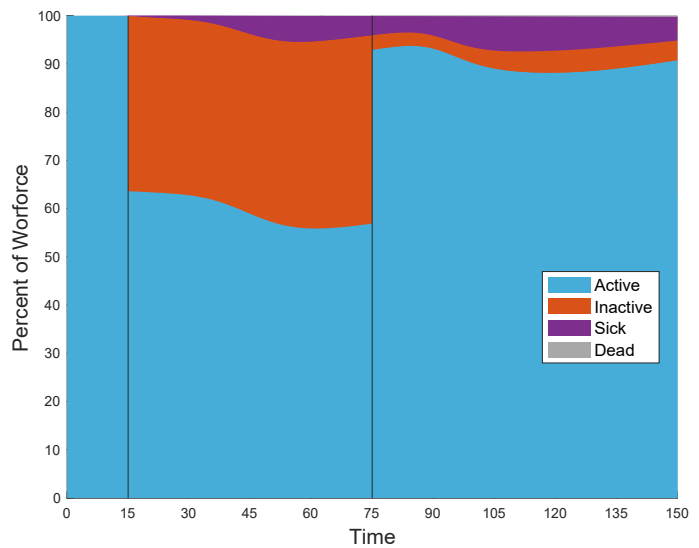

(c) Chicago, Healthcare Outcomes

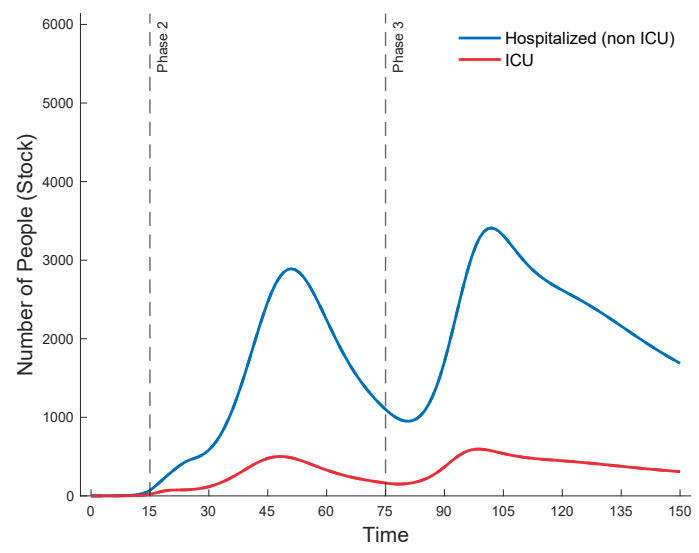

Note: The figure shows health and employment outcomes with No Policy in Phase 1, Essential Only in Phase 2 and Cautious Reopening in Phase 3 for the Chicago MSA, with contact matrices based on Replica data. The top left panel displays the percent of individuals of the local population that are infected and symptomatic and/or detected, and the number of deaths per 100,000s of population on the right vertical axis. The top right panel corresponds plots the share of workforce that is either active, inactive (due to quarantine, or not allowed to access the workplace and unable to work from home), sick, or deceased.

not account for hospital capacity and ICU capacity. This is an important venue for future work, since if our predicted hospitalization and ICU admissions exceed capacity, we expect death rates among symptomatic individuals to increase.

As shown in Figure 7b, when EO is imposed, only slightly more than $60 \%$ of individuals in Chicago are actively employed - either as workers in essential industries or by being able to work from home. This number decreases to $55 \%$ as individuals contract the disease, and either get sick, die, or become quarantined and unable to work from home. The CR policy- 
Figure 8: No Policy-Essential Only-Cautious Reopening Across MSAs

(a) Sacramento, Epidemic Outcomes

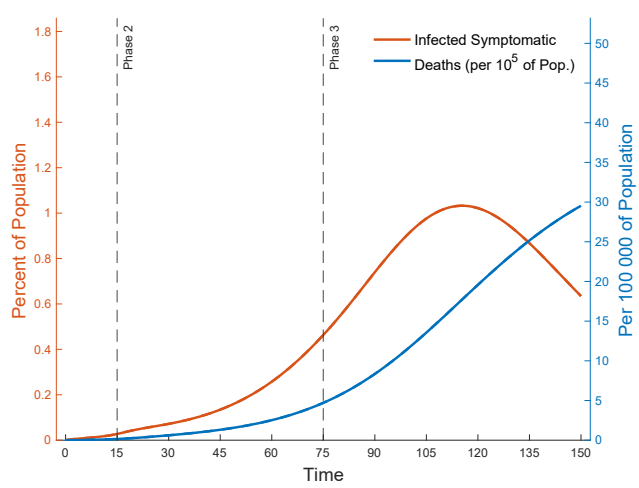

(c) Sacramento, Employment Outcomes

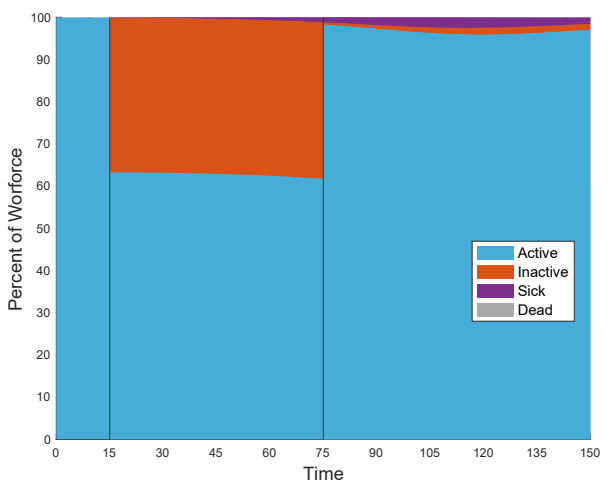

(e) Sacramento, Healthcare Outcomes

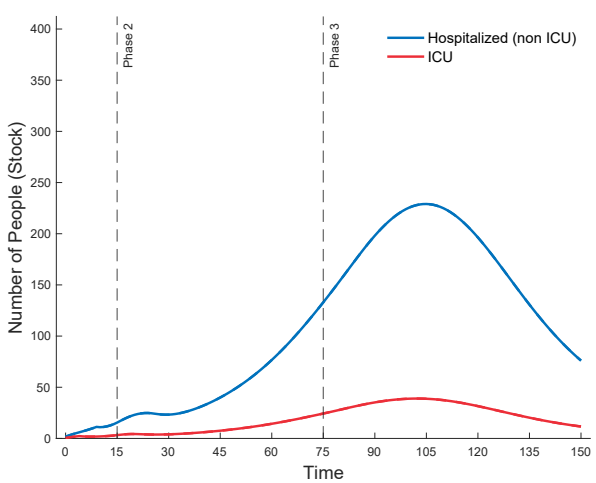

(b) New York, Epidemic Outcomes

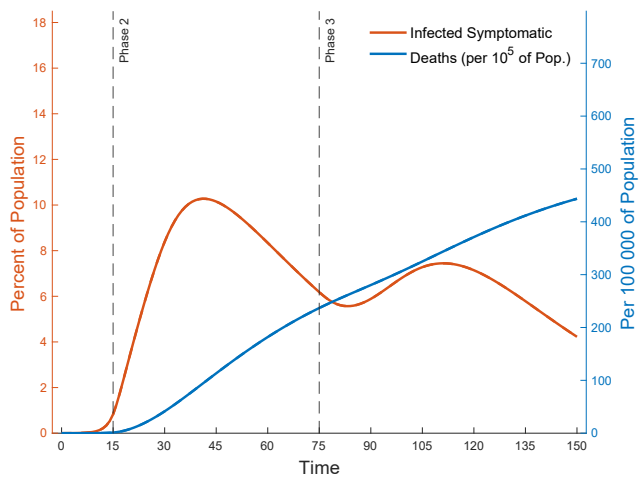

(d) New York, Employment Outcomes

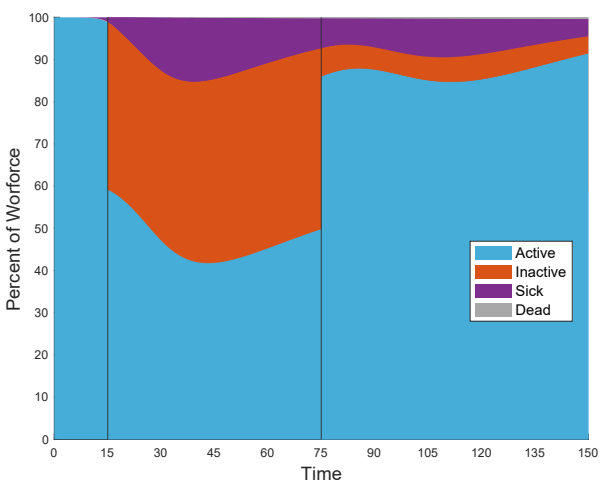

(f) New York, Healthcare Outcomes

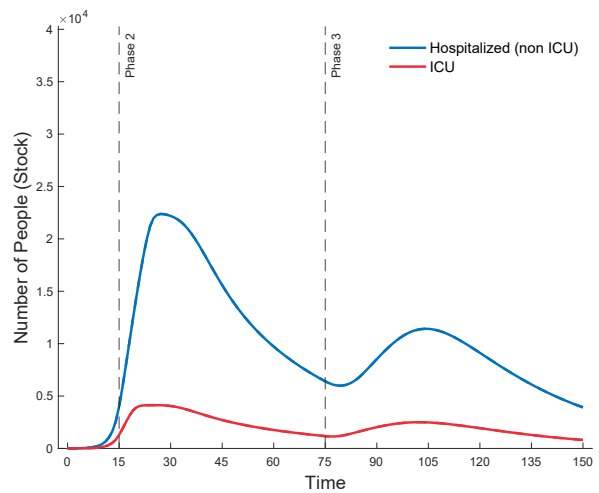

Note: The figure shows outcomes series with No Policy in Phase 1, Essential Only in Phase 2, and Cautious Reopening in Phase 3. The left column shows results for the Sacramento MSA, based on Replica data; the right column shows results for the New York MSA, based on FRED data. For further details refer to note to Figure 7 . 
under which the population returns to work-dramatically reduces the number of inactive individuals. However, even under CR, we see a new drop in employment 15 days into this phase, due to the rising number of sick, deceased, and quarantined individuals.

In Figure 8 we show the same outcome series for the Sacramento (Replica-based, like Chicago) and the New York (FRED-based) MSAs. These figures hint at considerable heterogeneity across the different regions. For example, in New York, the infection rate is relatively high under EO in Phase 2, but decreases steadily in Phase 3, even with the less stringent CR policy. Unlike Chicago, New York experiences a second peak of infection in Phase 3 that is $30 \%$ lower than the level of Phase 2.

As we showed in Section 3. Sacramento is less densely populated than Chicago and New York and features fewer individual contacts. As such, it does not exhibit a peak of infections in Phase 2, but infections, deaths, hospitalization, ICU admissions and the number of inactive individuals (due to illness, death, or quarantine) increase in Phase 3 , with a peak at $t=110$. However, it is important to notice how for this area, our model predicts an overall limited magnitude of the COVID-19 outbreak.

\subsection{Outcomes across Counterfactual Policy Sequences}

We can compute the time series introduced above for any policy sequence, as well as cumulative counts for each of our key outcomes. We now turn to comparing our baseline NP-EO-CR policy sequence with key alternatives. Our emphasis will be on sequences along which local authorities still impose NP in Phase 1, and EO in Phase 2. However, for Phase 3 we will consider not only $\mathrm{CR}$, but also the $60+, \mathrm{WFH}$, and AS policies as alternatives. For the sake of completeness, we also allow for counterfactual policies in Phase 2. However, as these refer to a counterfactual past, we consider them less relevant and do not emphasize their results.

For each alternative policy sequence, we compute cumulative outcome measures (total deaths, and total days of employment lost, etc.) at the level of each MSA. In Figure 9 we plot the cumulative number of deaths against days of employment lost, both in absolute magnitudes and relative to NP-EO-CR. The lower-left-most boundary of each graph may be read as a "death-to-employment frontier" 18 Triangular markers indicate cumulative outcomes for the sequences NP-EO-CR (baseline), NP-EO-60+, NP-EO-WFH, and NP-EO-AS in each MSA. Each marker is labeled with reference to the corresponding Phase 3 policy. Other (dotted) markers refer to changes in policy in Phase 2, so that NP-60+-CR, NP-AS-CR, NP-AS-WFH, etc. are also evaluated, for a total of 25 possible combinations across Phase 2 and Phase 3.

Figure 9 shows how our empirical framework delivers several insights, both within MSA and across MSAs. First, there is substantial heterogeneity in relative differences across policies in Sacramento as compared to Chicago and New York. In Sacramento, there is little difference across all of the policies for the total number of deaths due to COVID-19, both in relative and

\footnotetext{
${ }^{18}$ As noted in Acemoglu et al. (2020), this frontier may be useful in demonstrating the trade-offs between economic losses and public health losses from each policy.
} 
Figure 9: Health and Employment Outcomes for Counterfactual Policy Sequences

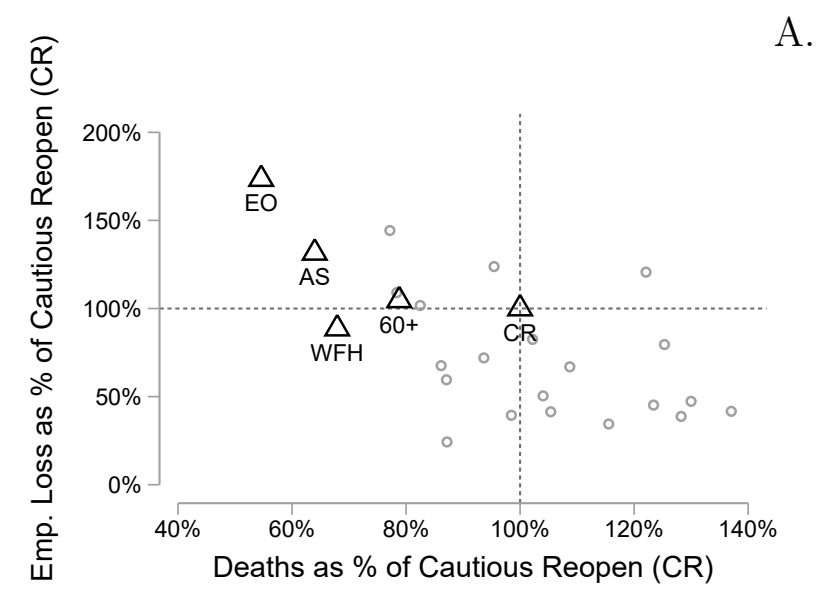

\author{
A. Chicago
}
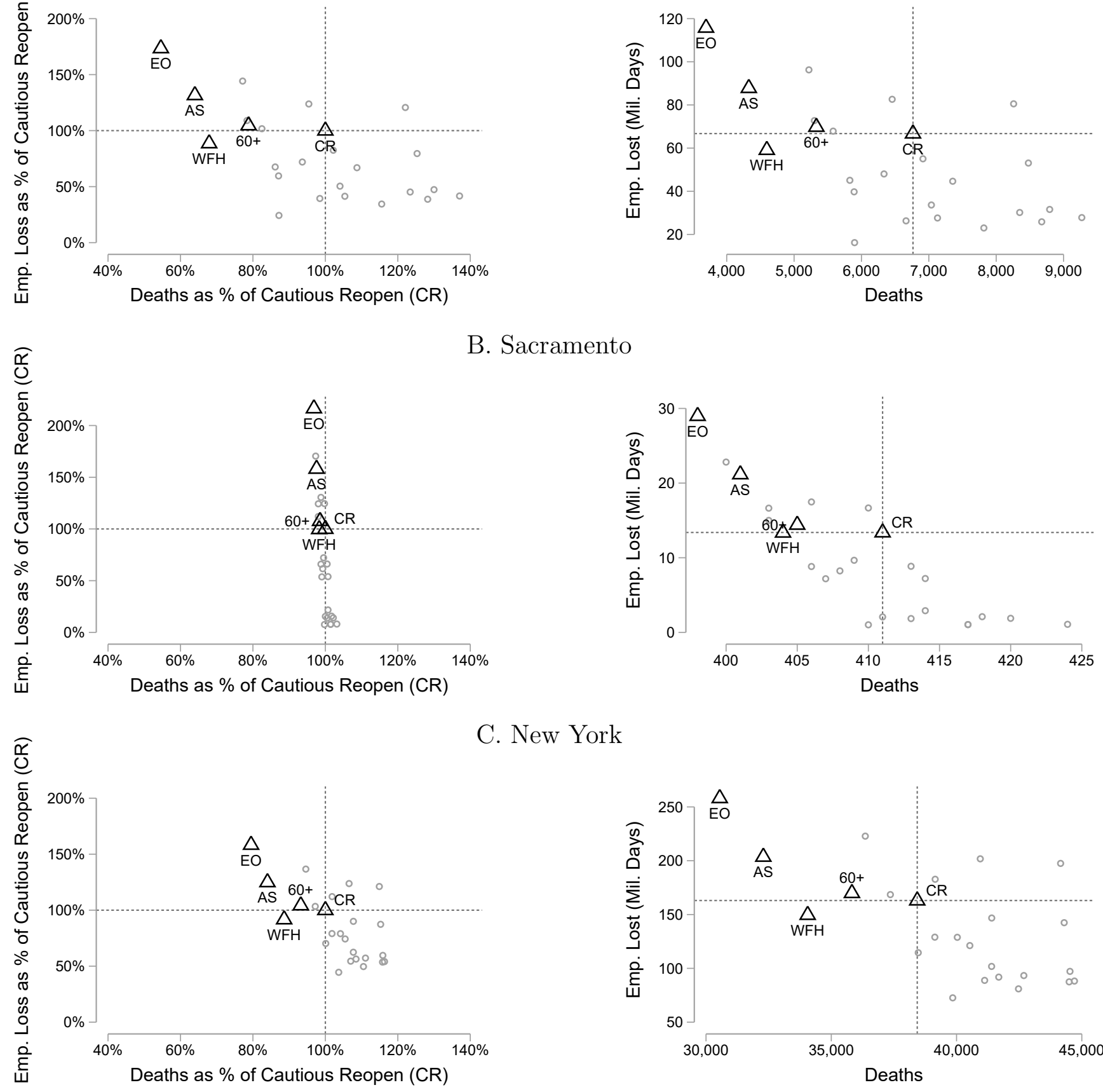

C. New York

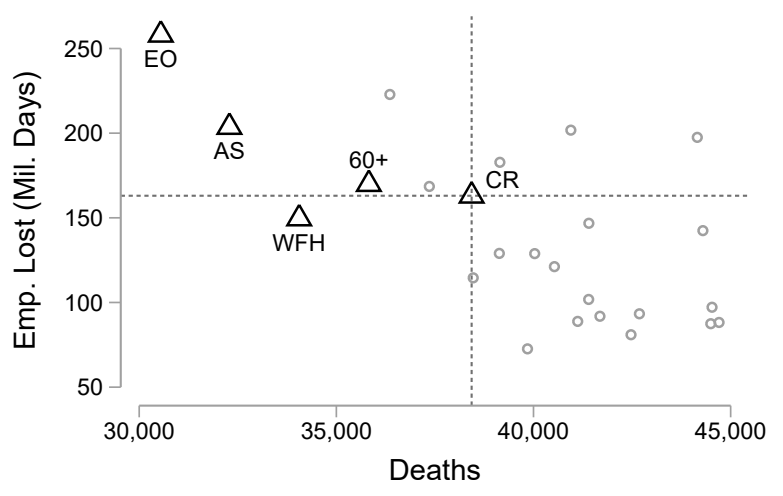

Note: Frontier: Employment versus lives lost. X-axis is the number of deaths by the end of Phase 3 and Y-axis is the employment days lost by the end of Phase 3. Presented as percentage relative to CR policy (left) and using raw counts (right). Triangles show predicted outcomes under a given policy in Phase 3. The hollow dots present a range of other potential outcomes that could have been achieved had alternate policies been pursed in Phase 2. 
absolute terms. This is a direct consequence of the smaller number of contacts. At the same time, as one would expect, the imposition of EO or AS in Phase 3 would be relatively more costly in terms of inducing employment losses compared to $\mathrm{CR}$, WFH or $60+$.

By contrast, for Chicago and New York, there seems to be substantial heterogeneity across policies in terms of outcomes along both mortality and employment. For example, in Chicago, any of the alternate policies that we consider would make a large difference in reducing deaths with respect to a cautious reopening. In fact, we predict that WFH in Phase 3 reduces the total number of deceased individuals (up to August 2nd, 2020) by almost 40\%, while maintaining a very similar level of employment losses.

Furthermore, for both Chicago and New York, isolating $60+$ in Phase 3 appears to be Pareto-dominated by imposing a work-from-home regime for the occupations that can be executed remotely (WFH). This suggests that simply isolating $60+$ year olds - who are at higher risk for severe cases of COVID-19 - is not as effective in reducing deaths as WFH even at a comparable level of unemployment.

We also find that, at the cost of slightly higher employment losses, alternating schedules (AS) is very effective in limiting deaths in both cities. If avoiding loss of lives was the only objective, AS would always rank second for Phase 3, topped only by prolonging the regime of EO into the summer. Note that here we are considering the "pure" AS policy, where all individuals work on alternate days. A hybrid solution where those who can work from home, do, and those who cannot, work on alternate days might potentially do even better than these two policies individually. Clearly, a deeper knowledge of the local economy, and the productive and organizational structure of local firms and establishments would be essential for a practical implementation of AS.

Although Chicago and New York are both large metropolitan areas, we observe notable differences in comparing their responses to different social distancing policies. As noted above, the two cities exhibit different disease dynamics: Chicago experiences a less-severe outbreak in Phase 1 and Phase 2, but remains more exposed to a severe outbreak in Phase 3. As a consequence, the model suggest that EO in New York was, in a sense, necessary. No alternative to EO in Phase 2 is Pareto-dominated by the baseline sequence NP-EO-CR. This is not the case in Chicago, where a slower epidemic outbreak in Phase 1 would have allowed imposing milder restrictions in Phase 2, but where the differences in outcomes across Phase 3 policies are now larger. Intuitively, the early path of the outbreak in Chicago left a larger number of susceptible individuals in Phase 3, making social distancing policies more salient. As such, while WFH reduces deaths by $40 \%$ in Chicago as compared to the baseline policy, in New York this number is about $20 \%$.

The heterogeneity of policy outcomes across areas is not only a function of differences in early disease dynamics and contact density across MSAs, but also of their different distributions of age-industry-occupation-comorbidity types, and cross-types encounters. As such, our model would be expected to produce different results for other cities, depending on their properties along these different dimensions. Finally, it is important to note that our New York results 
Table 3: Selected Counterfactual Outcomes for Chicago

\begin{tabular}{ccc|cccc}
\hline \multicolumn{3}{c|}{ Policy Sequence } & \multicolumn{4}{c}{ Cumulative Outcomes } \\
Phase 1 & Phase 2 & Phase 3 & $\begin{array}{c}\text { Deaths } \\
\text { (total) }\end{array}$ & $\begin{array}{c}\text { Hospital } \\
\text { (total days) }\end{array}$ & $\begin{array}{c}\text { ICU } \\
\text { (total days) }\end{array}$ & $\begin{array}{c}\text { Employment Loss } \\
\text { (1000s days) }\end{array}$ \\
\hline NP & EO & NP & 26890 & 1014953 & 165319 & 79193 \\
NP & EO & EO & 3693 & 120707 & 20216 & 115765 \\
NP & EO & CR & 6765 & 267024 & 46099 & 66728 \\
NP & EO & AS & 4328 & 158005 & 26538 & 87822 \\
NP & EO & WFH & 4595 & 168852 & 29224 & 59129 \\
NP & EO & $60+$ & 5331 & 236176 & 40870 & 69828 \\
NP & WFH & WFH & 5898 & 215612 & 39364 & 16199 \\
NP & $60+$ & $60+$ & 7038 & 301088 & 55082 & 33644 \\
NP & AS & AS & 5580 & 193112 & 32509 & 67873 \\
\hline
\end{tabular}

Note: The table shows all cumulative outcomes for Chicago across alternative policy sequences. Appendix Appendix $C$ contains a rich class of combinations for all MSAs, reporting cumulative outcomes and outcome dynamics.

are based on the FRED synthetic populations, while Sacramento and Chicago are based on Replica's proprietary data.

As a last note, it is important to reiterate that all of the results in this section (including CR) are assuming reduced neighborhood contact. We do not consider a full "back to normal," or formally NP in Phase 3. In Table 3, we summarize selected policy comparisons for Chicago, including our baseline policy sequence, amended to have NP in Phase 3. As demonstrated in the table, reopening fully without any restrictions to mobility and encounters of any sort would lead a much higher number of deaths, hospitalizations and ICU admissions. In Appendix C we report tabular and graphical output across a large number of policy combinations for each MSA 19

\subsection{Behavioral Changes, Masks, and Lower Contagion}

Mandatory policy intervention becomes less necessary if individuals respond more to the spread of disease by voluntarily taking preventive measures such as wearing masks and keeping $6 \mathrm{ft}$ apart. Such measures reduce the spread of respiratory droplets, and subsequently decrease the disease transmission rate (Prather et al., 2020).

In our model, the take-up of preventative measures in individual behavior corresponds to lowering the transmission probability upon contact, $\beta_{m t}$. For our main results, we employ two sets of calibrated $\beta_{m t}$ parameters for each MSA: an initial $\beta_{m}^{1}$ that governs transmission during Phase 1 , and a reduced $\beta_{m}^{2}$ that governs transmission during Phase 2 and Phase 3.

\footnotetext{
${ }^{19}$ The online material for this project (www.reopenmappingproject.com) will contain results for all MSAs in the US. These will be based on FRED data whenever and as long as Replica data are not yet available.
} 
Table 4: Sensitivity to Transmission Rate $\beta_{m t}$

\begin{tabular}{|c|c|c|c|c|c|c|c|c|}
\hline \multicolumn{2}{|l|}{ Policies } & Baseline & Phase $1 \beta_{m}^{1}$ & Phase $2 \beta_{m}^{2}$ & $0.9 \beta_{m t}$ & $0.75 \beta_{m t}$ & $0.5 \beta_{m t}$ & $0.25 \beta_{m t}$ \\
\hline & \multicolumn{8}{|c|}{ Chicago } \\
\hline $\mathrm{CR}$ & $\begin{array}{l}\text { Deaths } \\
\text { Emp. Loss (Mil. Days) }\end{array}$ & $\begin{array}{l}6,765 \\
66.7\end{array}$ & $\begin{array}{c}16,195 \\
83.4\end{array}$ & $\begin{array}{c}6,733 \\
67.0\end{array}$ & $\begin{array}{c}6,133 \\
65.4\end{array}$ & $\begin{array}{c}5,225 \\
63.2\end{array}$ & $\begin{array}{c}3,484 \\
57.9\end{array}$ & $\begin{array}{l}517 \\
46.9\end{array}$ \\
\hline \multirow[t]{2}{*}{ WFH } & $\begin{array}{l}\text { Deaths } \\
\text { Emp. Loss (Mil. Days) }\end{array}$ & $\begin{array}{c}4,595 \\
59.1\end{array}$ & $\begin{array}{c}12,101 \\
72.7\end{array}$ & $\begin{array}{l}4,434 \\
58.9\end{array}$ & $\begin{array}{c}4,140 \\
58.2\end{array}$ & $\begin{array}{l}3,515 \\
56.7\end{array}$ & $\begin{array}{c}2,325 \\
53.1\end{array}$ & $\begin{array}{l}481 \\
46.8\end{array}$ \\
\hline & \multicolumn{8}{|c|}{ Sacramento } \\
\hline $\mathrm{CR}$ & $\begin{array}{l}\text { Deaths } \\
\text { Emp. Loss (Mil. Days) }\end{array}$ & $\begin{array}{l}411 \\
13.4\end{array}$ & $\begin{array}{l}916 \\
14.7\end{array}$ & $\begin{array}{l}308 \\
13.0\end{array}$ & $\begin{array}{r}366 \\
13.2\end{array}$ & $\begin{array}{l}286 \\
12.9\end{array}$ & $\begin{array}{l}145 \\
12.4\end{array}$ & $\begin{array}{c}54 \\
12.0\end{array}$ \\
\hline \multirow[t]{2}{*}{ WFH } & $\begin{array}{l}\text { Deaths } \\
\text { Emp. Loss (Mil. Days) }\end{array}$ & $\begin{array}{l}404 \\
13.4\end{array}$ & $\begin{array}{c}793 \\
14.3\end{array}$ & $\begin{array}{c}302 \\
12.9\end{array}$ & $\begin{array}{c}361 \\
13.2\end{array}$ & $\begin{array}{r}283 \\
12.9\end{array}$ & $\begin{array}{l}143 \\
12.4\end{array}$ & $\begin{array}{c}54 \\
12.0\end{array}$ \\
\hline & \multicolumn{8}{|c|}{ New York } \\
\hline $\mathrm{CR}$ & $\begin{array}{l}\text { Deaths } \\
\text { Emp. Loss (Mil. Days) }\end{array}$ & $\begin{array}{c}38,431 \\
163.0\end{array}$ & $\begin{array}{l}58,222 \\
168.5\end{array}$ & $\begin{array}{c}34,328 \\
168.0\end{array}$ & $\begin{array}{c}33,621 \\
161.9\end{array}$ & $\begin{array}{c}26,442 \\
156.7\end{array}$ & $\begin{array}{c}14,658 \\
137.6\end{array}$ & $\begin{array}{l}4,399 \\
109.2\end{array}$ \\
\hline WFH & $\begin{array}{l}\text { Deaths } \\
\text { Emp. Loss (Mil. Days) }\end{array}$ & $\begin{array}{c}34,057 \\
149.6\end{array}$ & $\begin{array}{c}56,120 \\
161.9\end{array}$ & $\begin{array}{c}28,153 \\
149.8\end{array}$ & $\begin{array}{c}28,521 \\
146.3\end{array}$ & $\begin{array}{c}20,873 \\
139.4\end{array}$ & $\begin{array}{c}10,781 \\
124.7\end{array}$ & $\begin{array}{l}4,125 \\
108.2\end{array}$ \\
\hline
\end{tabular}

Note: The table shows how the simulated results change with transmission parameter $\beta_{m t}$. "Baseline" shows our main results using the calibrated parameters for Phase 1 and Phase 2. We then consider setting $\beta_{m t}$ to the calibrated value from Phase $\beta_{m}^{1}$ (for all periods), and to the calibrated value from Phase $2 \beta_{m}^{2}$. To demonstrate sensitivity to these values, we scale the baseline $\beta_{m t}$ values by a constant factor of 0.9, 0.75, 0.5, and 0.25. Each row shows deaths or employment loss in million-days for Sacramento, Chicago, and New York under two different reopening policies after May: Cautious Reopening (CR) and Work-From-Home if Possible (WFH).

Both of these parameters are calibrated with respect to observed death rates at the onset of the disease outbreak 20 We allow $\beta_{m t}$ to vary between phases during calibration to account for the possibility that behavioral responses became more rampant as lock-down policies proliferated. Although we do not impose any further restrictions on $\beta_{m}^{1}$ and $\beta_{m}^{2}$, we find that $\beta_{m}^{2}$ is substantially lower than $\beta_{m}^{1}$, suggesting that behavioral responses were indeed being taken up during Phase 2.

We test the sensitivity of our results to $\beta_{m t}$ in Table 4 . Focusing on the comparison between the $\mathrm{CR}$ and WFH policies, we compute deaths and employment loss under several alternative values of $\beta_{m t}$ in each city. Under the "Baseline" column, we report the death and employment loss numbers under our main setting with the different, calibrated $\beta_{m}^{1}$ and $\beta_{m}^{2}$ values. We then fix $\beta_{m t}=\beta_{m}^{1}$ and (in the next column) $\beta_{m t}=\beta_{m}^{2}$ for all periods $t$. Finally, we scale the baseline $\beta_{m t}$ with a constant factor of $0.9,0.75,0.5$, and 0.25 in each period.

As Table 4 shows, the relative performance of CR to WFH is largely preserved, even at a substantially reduced $\beta_{m t}$. However, the extent to which behavioral responses can substitute for policy restrictions also varies across regions. For instance, reducing the baseline $\beta_{m t}$ by $10 \%$ under CR leads to fewer deaths and less employment loss in Sacramento than switching to

\footnotetext{
${ }^{20}$ See Appendix $\mathrm{B}$ for details.
} 
WFH under the baseline $\beta_{m t}$. By contrast, in order to achieve a similar Pareto improvement in Chicago or New York, $\beta_{m t}$ would need to be reduced by over $25 \%$.

\section{Conclusions}

We develop an empirical model to study policy responses during a pandemic outbreak, with an immediate application to the COVID-19 pandemic. Our model is explicit about geographic and age-industry-comorbidity heterogeneity across individuals in their mobility and encounters patterns, their health risk specific to the disease, and their ability to work from home. This framework allows us to capture how different areas can expect different outcomes along alternative policy sequences. We inform our model with data on mobility, healthcare outcomes, and death series. This empirical framework is flexible in ingesting new, or different data, and it can be expanded to consider a richer set of policy alternatives.

Our results highlight the difference in trade-offs that different areas face when deciding how and when to re-open their local economies and relax social distancing measures. Areas that are hit early and have highly connected populations might expect a lower growth of the number of cases when relaxing social distance measures, while areas that had limited severity in the pandemic outbreak in early 2020 might expect cases to grow faster when these measures are relaxed and individual contacts grow, fueling contagion.

Policies by which individuals who can work from home do so, or policies by which school and work schedules are alternated (splitting classes and employees in groups) appear very promising for limiting contagion, while also containing employment losses. Behavioral responses in limiting contacts, and precautions such as wearing masks, can play a significant role in limiting the severity of the disease outbreaks.

Our current results are preliminary, and do not tackle directly a variety of important considerations. As mentioned above, we abstract away from hospital and ICU capacity in a given geographic area, and we do not yet observe healthcare outcomes broken down by geography. More data are available every week, and we are working to expand our analysis to encompass more of these details. The model is flexible, and designed to include these. Importantly, moreover, by looking at aggregate outcomes, we do not explore the heterogeneous and possibly distributional effects of policies across different types. The choice of policy affects who gets infected and which jobs are lost ${ }^{21}$ As such, our ongoing agenda is to include a richer description of socioeconomic status in the definition of a type, and investigate how our policies influence individuals in different income groups, races, and age groups. In terms of counterfactuals, our current results analyze one policy at a time. A natural next step is to combine policies (e.g., work-from-home if possible and isolate 60+) to locate their outcomes on the frontier. Finally, we have not directly looked into the effects of different policies on the broader production supply-chain network of the economy. Since shutting down a specific

\footnotetext{
${ }^{21}$ For instance, Mongey et al. (2020) show that low-income, less-educated workers are more economically vulnerable.
} 
sector has externalities on other sectors (Baqaee and Farhi, 2019), this is potentially a firstorder concern and we hope that mobility data can be used to investigate this angle as well.

\section{References}

Acemoglu, D., V. Chernozhukov, I. Werning, And M. D. Whinston (2020): "A multirisk sir model with optimally targeted lockdown," Tech. rep., National Bureau of Economic Research.

Aizawa, N. AND H. FAng (2020): "Equilibrium labor market search and health insurance reform," Journal of Political Economy, forthcoming.

Allcott, H., L. Boxell, J. Conway, , B. Ferguson, M. Gentzkow, and B. Goldman (2020a): "Economic and Health Impacts of Social Distancing Policies during the Coronavirus Pandemic," Working Paper.

Allcott, H., L. Boxell, J. Conway, M. Gentzkow, M. Thaler, and D. Y. Yang (2020b): "Polarization and public health: Partisan differences in social distancing during the Coronavirus pandemic," NBER Working Paper.

Azzimonti, M., A. Fogli, F. Perri, And M. Ponder (2020): "Social Distance Policies in ECON-EPI Networks," .

Baker, S. R., N. Bloom, S. J. Davis, K. J. Kost, M. C. Sammon, and T. Viratyosin (2020): "The unprecedented stock market impact of COVID-19," Tech. rep., National Bureau of Economic Research.

BAqAeE, D. R. AND E. FARHI (2019): "The macroeconomic impact of microeconomic shocks: beyond Hulten's Theorem," Econometrica, 87, 1155-1203.

Bartik, A. W., M. Bertrand, Z. B. Cullen, E. L. Glaeser, M. Luca, and C. T. StAnton (2020): "How are small businesses adjusting to covid-19? early evidence from a survey," Tech. rep., National Bureau of Economic Research.

Benzell, S., A. Collis, and C. Nicolaides (2020): "Rationing Social Contact During the COVID-19 Pandemic: Transmission Risk and Social Benefits of US Locations," Available at SSRN 3579678.

Berger, D. W., K. F. Herkenhoff, and S. Mongey (2020): "An seir infectious disease model with testing and conditional quarantine," Tech. rep., National Bureau of Economic Research.

Birge, J., O. Candogan, And Y. Feng (2020): "Controlling Epidemic Spread: Reducing Economic Losses with Targeted Closures," . 
Bollobás, B., S. Janson, And O. Riordan (2007): "The phase transition in inhomogeneous random graphs," Random Structures \& Algorithms, 31, 3-122.

Brzezinski, A., G. Deiana, V. Kecht, and D. Van Dijcke (2020): "The covid-19 pandemic: government vs. community action across the united states," Covid Economics: Vetted and Real-Time Papers, 7, 115-156.

Budish, E. B. (2020): " $R<1$ as an Economic Constraint: Can We 'Expand the Frontier' in the Fight Against Covid-19?" University of Chicago, Becker Friedman Institute for Economics Working Paper.

Cajka, J. C., P. C. Cooley, And W. D. Wheaton (2010): "Attribute assignment to a synthetic population in support of agent-based disease modeling," Methods report (RTI Press), 19, 1.

Chetty, R., J. N. Friedman, N. Hendren, and M. Stepner (2020): "Real-Time Economics: A New Platform to Track the Impacts of COVID-19 on People, Businesses, and Communities Using Private Sector Data," Tech. rep., Mimeo.

Dingel, J. I. And B. Neiman (2020): "How many jobs can be done at home?" Tech. rep., National Bureau of Economic Research.

Eubank, S., I. Eckstrand, B. Lewis, S. Venkatramanan, M. Marathe, and C. BarRETT (2020): "Commentary on Ferguson, et al., "Impact of Non-pharmaceutical Interventions (NPIs) to Reduce COVID-19 Mortality and Healthcare Demand"," Bulletin of Mathematical Biology, 82, 1-7.

Eubank, S., H. Guclu, V. A. Kumar, M. V. Marathe, A. Srinivasan, Z. Toroczkai, AND N. WANG (2004): "Modelling disease outbreaks in realistic urban social networks," Nature, 429, 180-184.

Farboodi, M., G. Jarosch, and R. Shimer (2020): "Internal and external effects of social distancing in a pandemic," Tech. rep., National Bureau of Economic Research.

Fernández-Villaverde, J. And C. I. Jones (2020): "Estimating and Simulating a SIRD Model of COVID-19 for Many Countries, States, and Cities," Tech. rep., National Bureau of Economic Research.

Fletcher, R. A., T. Matcham, M. Tibúrcio, A. Anisimovich, S. Jovanović, L. AlBergante, N. Lipunova, A. Hancock, L. Mackillop, L. Tarassenko, et Al. (2020): "Risk factors for clinical progression in patients with COVID-19: a retrospective study of electronic health record data in the United Kingdom," medRxiv. 
Gatto, M., E. Bertuzzo, L. Mari, S. Miccoli, L. Carraro, R. Casagrandi, and A. Rinaldo (2020): "Spread and dynamics of the COVID-19 epidemic in Italy: Effects of emergency containment measures," Proceedings of the National Academy of Sciences, 117, 10484-10491.

Glover, A., J. Heathcote, D. Krueger, And J.-V. Ríos-Rull (2020): "Health versus wealth: On the distributional effects of controlling a pandemic," Tech. rep., National Bureau of Economic Research.

Grefenstette, J. J., S. T. Brown, R. Rosenfeld, J. DePasse, N. T. Stone, P. C. Cooley, W. D. Wheaton, A. Fyshe, D. D. Galloway, A. Sriram, et Al. (2013): "FRED (A Framework for Reconstructing Epidemic Dynamics): an open-source software system for modeling infectious diseases and control strategies using census-based populations," BMC public health, 13, 940.

Grossman, M. (1972): "On the concept of health capital and the demand for health," Journal of Political Economy, 80, 223-255.

Gupta, S., T. D. Nguyen, F. L. Rojas, S. Raman, B. Lee, A. Bento, K. I. Simon, AND C. Wing (2020): "Tracking public and private response to the covid-19 epidemic: Evidence from state and local government actions," Tech. rep., National Bureau of Economic Research.

Loertscher, S. And E. Muir (2020): "Road to Recovery: From Extinguishing An Epidemic to Managing It," Available at SSRN $358499 \%$.

McCombs, A. AND C. Kadelka (2020): "A model-based evaluation of the efficacy of COVID-19 social distancing, testing and hospital triage policies," medRxiv.

Mizumoto, K., K. Kagaya, A. Zarebski, and G. Chowell (2020): "Estimating the asymptomatic proportion of coronavirus disease 2019 (COVID-19) cases on board the Diamond Princess cruise ship, Yokohama, Japan, 2020," Eurosurveillance, 25, 2000180.

Mongey, S., L. Pilossoph, And A. Weinberg (2020): "Which workers bear the burden of social distancing policies?" Tech. rep., National Bureau of Economic Research.

Moran, R. J., E. D. Fagerholm, M. Cullen, J. Daunizeau, M. P. Richardson, S. Williams, F. Turkheimer, R. Leech, and K. J. Friston (2020): "Estimating required'lockdown'cycles before immunity to SARS-CoV-2: model-based analyses of susceptible population sizes,'S0', in seven European countries, including the UK and Ireland [version 1; peer review: awaiting peer review]," Wellcome Open Research, 5.

Novel, C. P. E. R. E. ET AL. (2020): "The epidemiological characteristics of an outbreak of 2019 novel coronavirus diseases (COVID-19) in China," Zhonghua liu xing bing xue za zhi= Zhonghua liuxingbingxue zazhi, 41, 145. 
Prather, K. A., C. C. Wang, and R. T. Schooley (2020): "Reducing transmission of SARS-CoV-2," Science.

Prem, K., Y. Liu, T. W. Russell, A. J. Kucharski, R. M. Eggo, N. Davies, S. Flasche, S. Clifford, C. A. Pearson, J. D. Munday, et Al. (2020): "The effect of control strategies to reduce social mixing on outcomes of the COVID-19 epidemic in Wuhan, China: a modelling study," The Lancet Public Health.

RuAn, S. (2020): "Likelihood of survival of coronavirus disease 2019," The Lancet Infectious Diseases, 20, 630-631.

Soriano-Paños, D., J. H. Arias-Castro, A. Reyna-Lara, H. J. Martínez, S. Meloni, And J. Gómez-Gardenes (2020): "Vector-borne epidemics driven by human mobility," Physical Review Research, 2, 013312.

Stock, J. H. (2020): "Reopening the Coronavirus-Closed Economy," .

Verity, R., L. C. Okell, I. Dorigatti, P. Winskill, C. Whittaker, N. Imai, G. Cuomo-Dannenburg, H. Thompson, P. G. Walker, H. Fu, et Al. (2020): "Estimates of the severity of coronavirus disease 2019: a model-based analysis," The Lancet infectious diseases.

Villas-Boas, S. B., J. Sears, M. Villas-Boas, and V. Villas-Boas (2020): “Are We\# StayingHome to Flatten the Curve?" .

Will, M., J. Groeneveld, K. Frank, And B. Müller (2020): "Combining social network analysis and agent-based modelling to explore dynamics of human interaction: A review," Socio-Environmental Systems Modelling, 2, 16325-16325. 


\section{Appendices}

\section{A Details on Data Sources}

\section{A.1 Replica Data}

\section{A.1.1 Building a Synthetic City}

Replica is a transportation planning company spun out of Alphabet in 2019 22 For each city, Replica generates a synthetic representation of the activities and movement of residents, visitors, and commercial vehicle fleets for a representative week during a quarter. Replica ingests data from four primary sources in order to create these synthetic cities: 1) public use census data; 2) granular location data for a sample of devices, from both GPS pings and triangulation between cellular towers; 3) proprietary data describing features of various points of interest (POIs), such as their exact location, brand, and industry; and 4) data from public agencies on traffic and transit usage ('ground truth'), often sourced from sensors placed on roads and public transit turnstiles.

There are four major steps to constructing a Replica city.

1. Build a synthetic population with people and households that with demographics that mirror the joint-distributions of the real population's demographics and household structure.

2. Assign each person a home and, where applicable, a work or school location.

3. Generate a 'typical day' of mobility for each person, consisting of an activity sequence (e.g., [home, work, eat, work, shop, home]), a location for each activity, and travel modes between activities. Figure Figure A.1 provides an illustrative example of an activity sequence for a single individual.

- Activities are produced using generative models trained on GPS and telco devices. Location data from GPS traces are very spatially accurate, but cover only a small sample of devices, whereas location data from telco devices are less spatially accurate (as they depend on triangulation between cellular towers), but cover upwards of $20 \%$ of the population.

- There are three generative models: the first determines the activity sequence for the day, the second determines exact locations of the non-home/work activities, and the final assigns travel modes between each activity based on the origin/destination and available travel modes.

- Mobility 'personas' comprising of the above generative models are matched to the synthetic population based primarily on their home, work, and school locations, plus

\footnotetext{
${ }^{22}$ For more information, visit their website: https://replicahq.com/
} 
some model that infers their age, employment status, vehicle ownership, and other demographics.

- Residents under 5 are not modeled. Residents under 16 are not in the labor force and only go to school and their home. College students may go to school in addition to work, shop, and other standard activities.

4. Calibrate using various 'ground truth' data sources provided by each city, including transit usage, cars on highways (from sensors), and others.

The process accomplishes two primary goals: 1) accurately capture the true movement of individuals in a city and 2) protect the privacy of those in the underlying location data. No individual in the synthetic population can me matched to an exact device.

Each Replica city is built separately, using all available data for a given quarter. Generating a new Replica city requires significant data gathering efforts and computational time. As such, coverage is currently limited to a handful of US cities.

Figure A.1: Illustration of an activity sequence

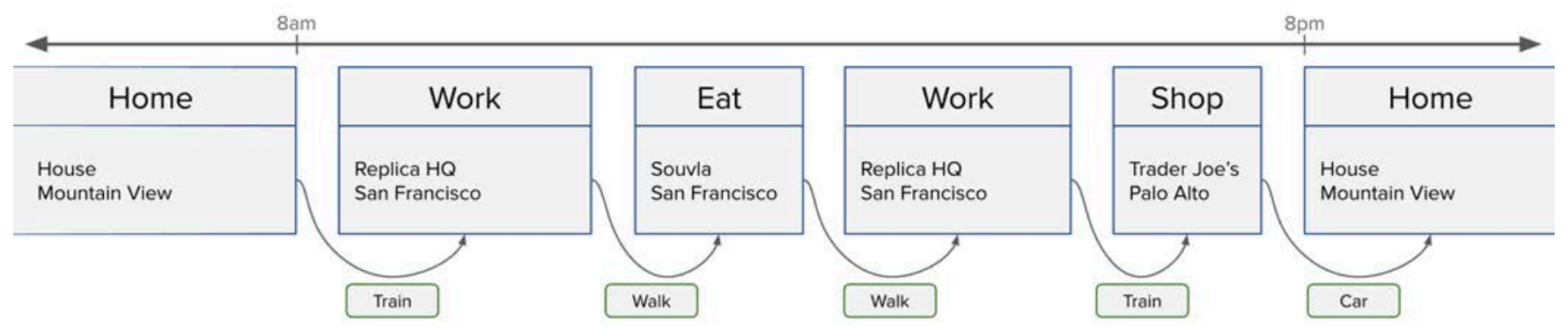

\section{A.1.2 Defining a Graph of Interactions}

We construct the graph of social interactions in a city using the locations and times of activities for each person. In this graph, people are nodes and edges are formed between people whenever they are at the same location at the same time. Locations are varied in sizes. Edges can be formed between people doing two different activities, e.g., between a customer shopping and an employee working at the same store.

'Core' and 'Donut' Geographies: The graph is built for the full Replica city, which includes outer areas. However, degree distributions for people who live on the geographic boundary of the Replica city would be inaccurate; interactions with those who live on the other side would not be observed. To help account for this, our results use as focal individuals only those living in the 'core' counties of these areas (Cook County and Sacramento County). Edges formed with those outside the core (people in the 'donut' region) still count towards, for example, a core person's degree distribution and contacts. 
Workplaces: Employed residents are assigned industries and accompanying workplaces. For customer-facing industries, we assign workers to workplaces corresponding to points of interest (POIs) of the corresponding industry (e.g., a 'Retail Trade' worker may be assigned to work at a Walmart). POIs are not available for all workplaces; while a Walmart or dentist's office would appear in our POIs data, a hedge fund's office would not. For industries that encompass firms that are either infrequently visited by customers or absent from our data on POIs - for example, construction and manufacturing - we divide workers into workplaces by sampling from a distribution of workplaces sizes.

The full procedure for assigning a given employed resident a specific workplaces is as follows:

1. Take all employed workers in a Census Block Group (CBG), industry (NAICS 2-digit) pair.

2. For those are in industries that are customer-facing and have data on POIs ${ }^{23}$ randomly assign POIs of the same industry as the worker as workplaces, weighting by the number of visits to that POI relative to other POIs in that CBG-NAICs. So a Walmart that receives $50 \%$ of all retail traffic in a given $\mathrm{CBG}$ will have $50 \%$ of the retail workers in that CBG assigned to it as 'employees'

3. For workers in other industries ${ }^{24}$ we divide up large groups of workers in a CBG-NAICS into smaller workplaces. For CBG-NAICS that have $>150$ employees, we break them into groups where a worker's group number is sampled from a Poisson distribution. The rate parameter is chosen so that the average group size is 75 employees ${ }^{25}$

4. For POIs with a large number of employees assigned via the above method, we break into working divisions using the same parameters.

5. For workers, edges are formed between coworkers at work simultaneously and - when the workplace has a POI-between workers and customers who arrive while the worker is present. Worker-to-worker links for large workplaces must be within the same 'group,' but worker-to-customer do not.

Schools: Students are assigned schools by Replica; however, many schools are unrealistically large (e.g., a college campus would be considered a single school). To help address this issue, we break up schools into classes in a similar manner to how we divide up large workplaces, but with different parameters. In schools with over 100 students, each student is assigned a class where class number is sampled from a Poisson distribution where the rate parameter is calibrated to produces average class sizes of 50 students. All students within the same

\footnotetext{
${ }^{23}$ The following industries are matched to POIs: 'Wholesale Trade', 'Retail Trade', 'Information', 'Finance and Insurance', 'Real Estate and Rental and Leasing', 'Professional, Scientific, and Technical Services', 'Health Care and Social Assistance', 'Arts, Entertainment, and Recreation', 'Accommodation and Food Services'

${ }^{24}$ These include: 'Construction', 'Manufacturing', 'Transportation and Warehousing', 'Administrative and Support', 'Educational Services', 'Other', 'Public Administration'.

${ }^{25}$ In future versions, these parameters will be calibrated to match the true distribution of firm sizes in a given industry-location.
} 
We are currently investigating data on both firm size (by industry) and average class size for schools within a given Census Tract. With this data, we can calibrate the distribution of workplaces groups and school classes to better match the true distribution.

Households: Members of the same household are all connected, with a single contact of activity type 'home.'

\section{A.1.3 Comparing Replica and ACS Populations}

We compare Replica's synthetic population to the 2018 5-year ACS. Figures Figure A.2a and Figure A.2b plot the distributions of ages in each population for Chicago (Cook County) and Sacramento (Sacramento County). Figures Figure A.2c and Figure A.2d plot the distributions of industries for workers. Figures Figure A.2e and Figure A.2f plot the distribution of ageindustry types. The age distributions are nearly identical, but Replica consistently undermatches the number of people in a given industry by a small margin.

\section{A.2 FRED Data}

\section{A.2.1 Synthetic Population in FRED}

FRED (a Framework for Reconstructing Epidemic Dynamics) is a freely available open-source agent-based modeling system for studying pandemic influenza. FRED uses open-access censusbased synthetic populations developed by RTI International, which capture the demographic and geographic heterogeneities, including realistic household, school, and workplace social networks.

We use the 2010 version of synthetic population accessed from FRED website (https:// fred.publichealth.pitt.edu/syn_pops). The following steps summarize the construction of the synthetic population at a high level. Please refer to Cajka et al. (2010) for more detailed descriptions.

1. The synthetic population uses an iterative fitting method to generate a population from US Census Bureau's Public Use Microdata files (PUMS) and aggregates data from the 2005-2009 American Community Survey (ACS) 5-year sample.

2. Determines the number of household in each census block group based on Summary File 3 (SF3) Data from Census, and randomly generates a GIS point location for each household within each census block.

3. Assigns school-age individuals to schools, using National Center for Education Statistics (NCES) Public School Data for 2005-2006, US Census Bureau 2000 TIGER road network data, and private school information from Schoolinformation.com. The allocation to public school is based on a minimum path algorithm that assigns a student to the closest school that has capacity for his/her grade level. The remaining school-age individuals are assigned to private schools or home school. 
Figure A.2: Comparing ACS and Replica Populations

(a) Chicago: Age

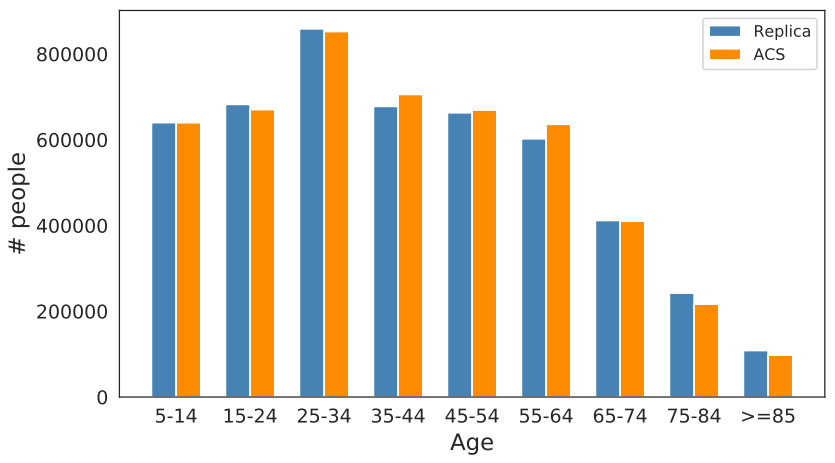

(c) Chicago: Industry

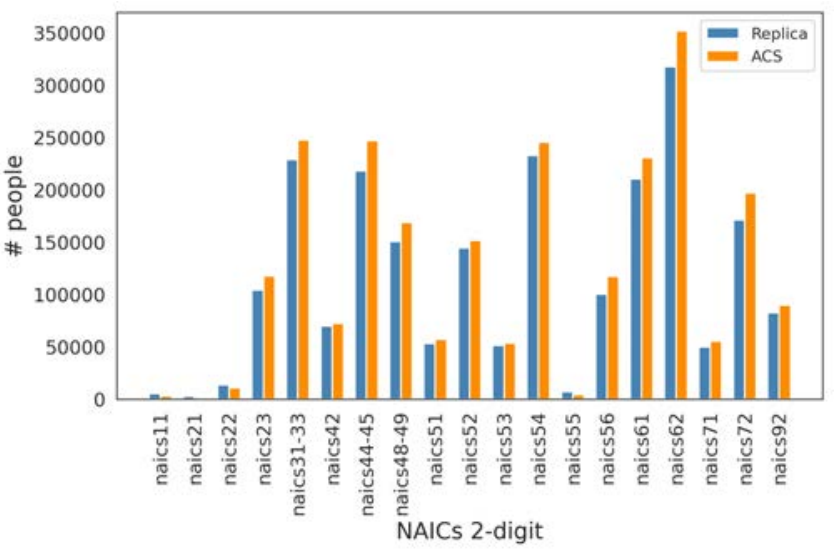

(e) Chicago: Age-Industry

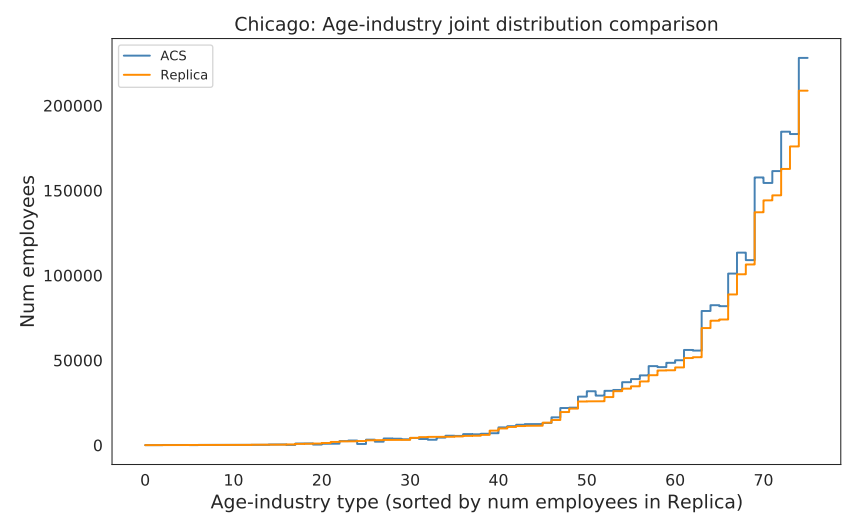

(b) Sacramento: Age

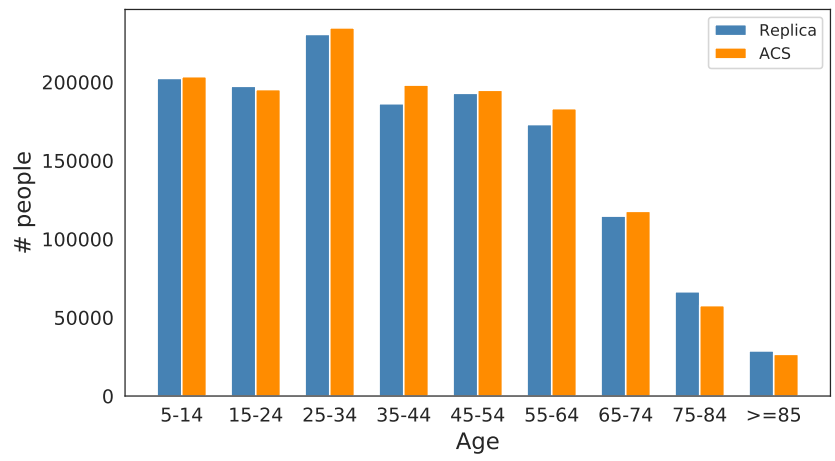

(d) Sacramento: Industry

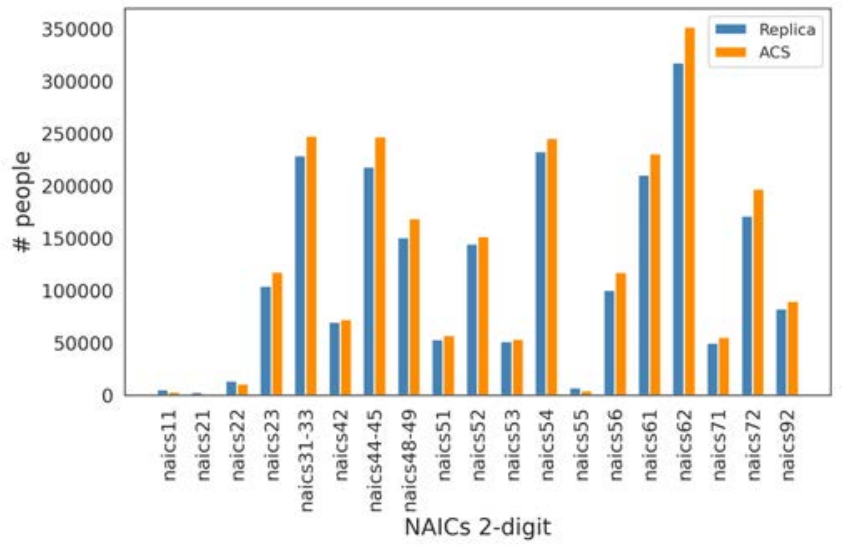

(f) Sacramento: Age-Industry

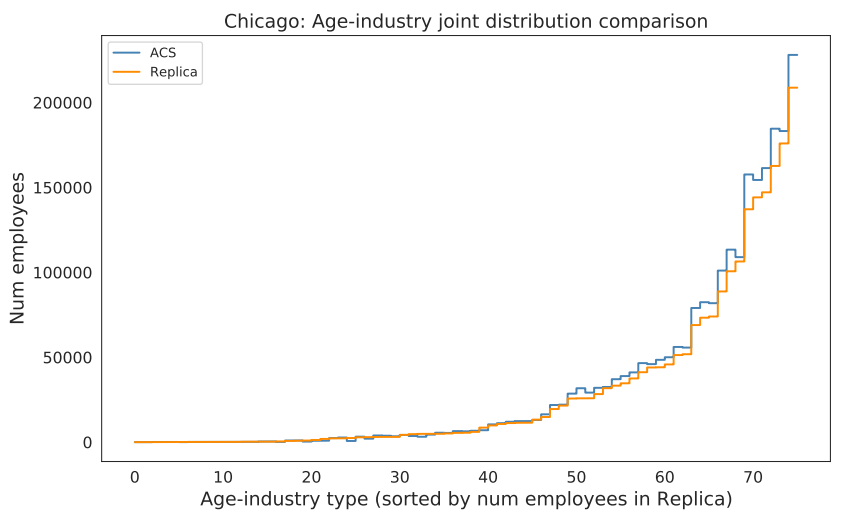

Note: These Figures compare the distribution of age, industry, and age-industry in both Replica and the 2018 5-year ACS populations for Chicago and Sacramento. 
4. Assigns non-school-age population to workplaces, using US Census Bureau Special Tabulation Product 64 (STP64) and 2006 InfoUSA Business Counts, following a two-stage method. The first stage assigns workers to a Census tract for work. The second stage creates individual workplace locations within each Census tract and assigns workers to specific workplaces.

\section{A.2.2 Defining a Graph of Interactions}

Using the synthetic populations within each MSA, we construct the graph of social interactions using locations and assignments of households, schools, and workplaces.

Workplaces: Individuals contact colleagues in the same assigned synthetic workplace. In the synthetic population, the size of workplaces can be large. So we break workplaces into subgroups, using a similar procedure as described in Appendix A.1. The size of subgroups is sampled from a Poission distribution and the rate parameter is an exponential transformation of the size of synthetic workplace relative to the average size in each MSA 26

Since industry is a key source of heterogeneity we consider, we assign a 2 digit NAICS code to each synthetic workplace. We take the 2007 County Business Pattern, which includes the number of establishments in each county $\times$ NAICS- $2 \times$ size cohort ${ }^{27}$. Using the synthetic workplaces and assigned workers, we group workplaces in each county into the same size cohorts. Then, we randomly assign each workplace an NAIC 2 code assuming a multinomial distribution.

Schools: Students interact with other students assigned to the same synthetic school. We divide up schools similar to the procedure for workplaces.

Neighborhoods: We assume individuals form contacts with others living in the same neighborhood. A neighborhood covers the same longitude and latitude rounded to the second decimal points, which is a squared block of slightly more than $1 \mathrm{~km}^{2}$. According to this definition, a neighborhood block can include many people in metropolitan areas, and we divide up neighborhood blocks similar to the procedure for workplaces and schools.

Households: We assume individuals contact all other members in the same household.

\section{A.3 Healthcare Data}

The sample of confirmed 3454 confirmed cases from the COVID-19 Research Database is constructed as follows. First, we consider all individuals for which we observe electronic medical

\footnotetext{
${ }^{26}$ The rate parameter is equal to $4+(100-4) \times(1-\exp (-\alpha \times$ workplace size/avg neighborhood size $))$ where $\alpha$ is a parameter we calibrate.

${ }^{27}$ Size is in terms of number of employees, with the following cohorts ¡5, 5-9, 10-19 20-49, 50-99, 100-249, 250-499, 500-999, 1000-1499, 1500-2499, 2500-4999, 5000+
} 
records. We identify our sample considering the official guidelines for ICD10 coding, and in particular we include all individuals for which we observe one of the following ICD10 diagnostic codes between Jan-1-2020 and May-1-2020: U04.9, B34.2, U07.1, U07.2.

Centers for Disease Control and Prevention (CDC) guidelines before April 2020, the official codes for COVID-19 were

- U04.9 Severe acute respiratory syndrome [SARS], unspecified;

- B34.2 Coronavirus infection, unspecified site.

After April 2020 the CDC guidelines included new WHO recommendations to exclude COVID diagnoses from using the above codes, and instead adopt:

- U07.1 COVID 19, virus identified Description: "Use this code when COVID-19 has been confirmed by laboratory testing irrespective of severity of clinical signs or symptoms. Use additional code, if desired, to identify pneumonia or other manifestations";

- U07.2: COVID 19, virus not identified. Description: "Use this code when COVID-19 is diagnosed clinically or epidemiologically but laboratory testing is inconclusive or not available.Use additional code, if desired, to identify pneumonia or other manifestations".

For these individuals, we consider all procedures observed between 25 days prior and 20 days after the diagnosis. We then identify hospitalizations using CPT codes 99221-99239, and ICU admissions using CPT codes 99291 and 99292.

To identify diabetes, we use ICD10 E11, ICD9 250, and SNOMED 7321109. To identify obesity, we use ICD10 E66, ICD9 278, and SNOMED 162864005.

\section{A.4 Ability to Work from Home (WFH)}

\section{A.4.1 Ability to WFH by Industry}

Notation We index SOC (finer) occupations $l \in\{1 \ldots, L\}$ : used by SOC and OES. We index 3-digit OCC (coarser) occupations $\widetilde{j} \in\{1, \ldots, \widetilde{J}\}$ : these are the 2010 Census OCC codes used by the 2018 ACS. We index 2-digit OCC occupations $j \in\{1, \ldots, J\}$. We index $\mathrm{O}^{*} \mathrm{NET}$ occupation characteristics $k=1, \ldots, K$. We index 2-digit NAICS codes $i \in\{1, \ldots, I\}$

Data construction We then construct the likelihood that a 2 digit occupation is teleworkable (i.e. work-from-home), $P$ (teleworkable $\mid j$ ) as follows

1. Use data from $\mathrm{O}^{*} \mathrm{NET}$ on 17 job characteristics. Each characteristic $m_{k l} \in[1,5]$

(a) Work Contexts: 1. Electronic Mail Use (reverse) 2. Outdoors, Exposed to Weather; 3. Outdoors, Under Cover; 4. Deal With Physically Aggressive People; 5. Wear Specialized Protective or Safety Equipment such as Breathing Apparatus, Safety Harness, Full Protection Suits, or Radiation Protection; 6. Wear Common Protective or Safety Equipment such as Breathing Apparatus Safety Harness, Full 
Protection Suits, or Radiation Protection; 7. Spend Time Walking and Running; 8. Exposed to Minor Burns, Cuts, Bites, or Stings; 9. Exposed to Disease or Infections.

(b) Work Activities: 1. Performing General Physical Activities; 2. Handling and Moving Objects; 3.Controlling Machines and Processes; 4. Operating Vehicles, Mechanized Devices, or Equipment; 5. Performing for or Working Directly with the Public; 6. Inspecting Equipment, Structures, or Material; 7. Repairing and Maintaining Electronic Equipment; 8. Repairing and Maintaining Mechanical Equipment

2. Use employment data $n_{l}$ from the national OES.

3. Collapse $\mathrm{O}^{*} \mathrm{NET}$ characteristics to the OCC level employment using employment weights from the national OES

$$
\begin{aligned}
\bar{m}_{j k} & :=\sum_{l \in j} w_{l} m_{k l} \\
w_{l} & :=\frac{n_{l}}{\sum_{l^{\prime} \in \tilde{j}} n_{l^{\prime}}}
\end{aligned}
$$

4. For each of $17 \mathrm{O}^{*} \mathrm{NET}$ attributes, we convert each into binary variables $m_{j k}^{*} \in\{0,1\}$ based on whether the raw measure $\bar{m}_{\widetilde{j} k} \geq 3.5$.

5. Following Dingel and Neiman (2020), we call a 3-digit occupation $\widetilde{j}$ teleworkable if and only if $\sum_{k \in K} m_{\tilde{j} k}^{*}=0$. In other words, we say an occupation is not teleworkable if any of the above work activities are important to the occupation.

$$
\text { teleworkable }_{\tilde{j}}:= \begin{cases}1 & \sum_{k \in K} m_{\tilde{j} k}^{*}=0 \\ 0 & \sum_{k \in K} m_{\tilde{j} k}^{*}>0\end{cases}
$$

6. We compute the share of a 2-digit occupation $j$ that is teleworkable using national employment weights from the OES.

$$
\begin{aligned}
P(\text { teleworkable } \mid j) & :=\sum_{\widetilde{j} \in j} \text { teleworkable } e_{\tilde{j}} \omega_{\tilde{j}} \\
\omega_{\widetilde{j}} & :=\frac{n_{\widetilde{j}}}{\sum_{\widetilde{j} \in j} n_{\widetilde{j}}}
\end{aligned}
$$

\section{A.4.2 Ability to WFH by Industry and Age}

We want to know, conditional on age $a$ and industry $i$, the share of workers who can workfrom-home $P(W F H \mid i, a)$. Our age bins of interest are [5, 17], [18, 49], [50, 64], 65+.

From the 2018 ACS 5-year, we compute employment in each occupation conditional on industry and age $P(j \mid i, a)$. From $\mathrm{O}^{*} \mathrm{NET}$ we know $P$ (teleworkable $\mid j$ ), the likelihood that 
Table A.1: Teleworkable share by Occupation

\begin{tabular}{lc}
\hline \hline Occupation & Share teleworkable \\
\hline Education & 0.972 \\
Legal & 0.957 \\
Computer/Math & 0.916 \\
Business/Financial & 0.804 \\
Architecture/Engineering & 0.578 \\
Science & 0.544 \\
Office/Admin & 0.519 \\
Management & 0.474 \\
Entertainment/Media & 0.436 \\
Sales & 0.263 \\
Personal care & 0.104 \\
Healthcare tech. & 0.041 \\
Healthcare supp. & 0.013 \\
Protection services & 0.009 \\
Production & 0.006 \\
Install/Maintenance/Repair & 0.006 \\
Farm/Fish/Forest & 0 \\
Material moving & 0 \\
Building maintenance & 0 \\
Construction/Extraction & 0 \\
Food prep. & 0 \\
Community/Social & 0 \\
Transport & 0 \\
\hline
\end{tabular}


an individual in occupation $j$ can work-from-home. With an independence assumption, this allows us to compute the probability a worker can work-from-home conditional on age and industry.

$$
P(\text { teleworkable } \mid i, a)=\sum_{j \in J} P(\text { teleworkable } \mid j) \times P(j \mid i, a)
$$

For example, notice that among workers in industry 11, Agriculture, Forestry, Fishing, and Farming, younger workers are less likely to be employed in a teleworkable occupation than older workers.

Figure A.3: Share of age x industry bins employed in teleworkable occupations
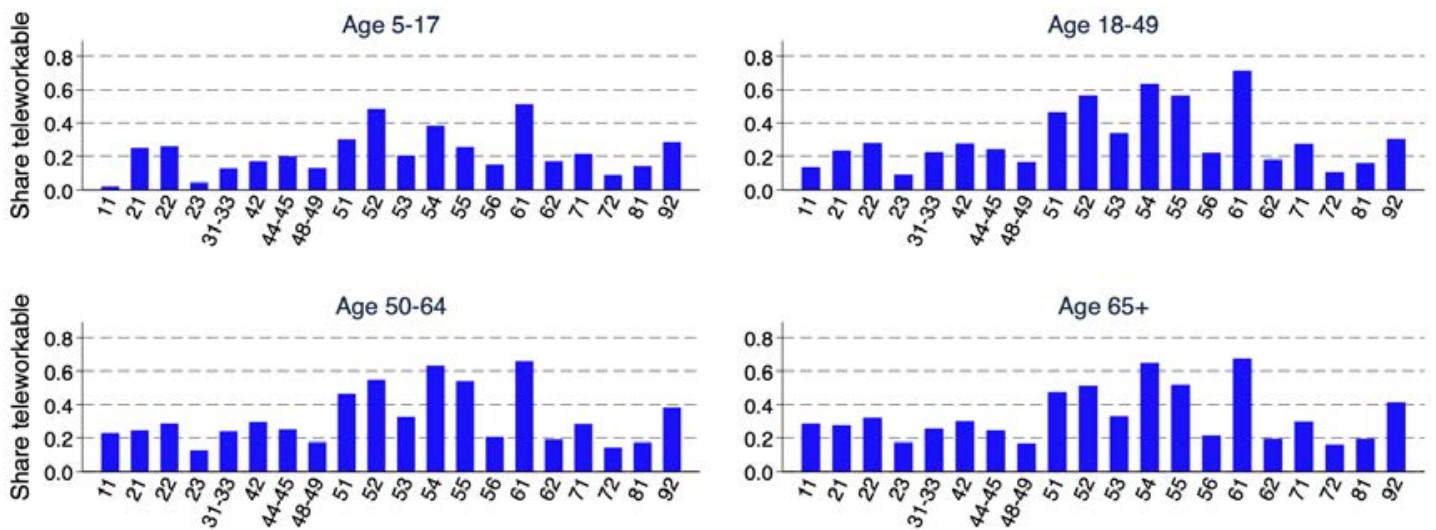

Note: This figure compares groups of 2 digit NAICS industries and age groups by employment in teleworkable occupations. To construct this figure, we follow Dingel and Neiman (2020) and Mongey et. al. (2020) by labeling occupations as non-teleworkable if they are sufficiently intensive in $O^{*} N E T$ activities that prevent the occupation from being performed remotely. Occupations are teleworkable otherwise. We compute the probability of being employed in a teleworkable occupation conditional on industry and age as $P($ teleworkable $\mid i, a)=\sum_{j \in J} P($ teleworkable $\mid j) \times$ $P(j \mid i, a)$. We use data from the 2018 ACS 5-year and $O * N E T$.

\section{B Calibration of Contagion Probability, Model Fit}

The chosen definition of a contact, and the specific nature of the available synthetic population, affect the magnitude and interpretation of the parameter governing contagion given contact. In other words, our parameter $\beta_{m t}$ for the "probability of infection upon a Replica contact in MSA $m$ in day $t$ " is to be interpreted as the "probability of infection upon face-to-face contact in MSA $m$ in day $t$ " multiplied by the "probability of a face-to-face contact in MSA $m$ in day $t$ upon a Replica contact in the same location-day".

To account for this, we estimate the probability of contagion given contact via indirect inference matching the model-predicted death series (using the parameters in Table 2) to the cumulative deaths at daily frequency from Johns Hopkins University Center for Systems 
Science and Engineering ${ }^{28}$ This is similar to Fernández-Villaverde and Jones (2020).

Currently, for each MSA $m$ we estimate three parameters: the transmission rate $\beta_{m}^{1}$ applying to Phase 1 from $t=0$ to $t=15$, the transmission rate $\beta_{m}^{2}$ applying to Phases 2 and 3 for $t>15$, and the initial condition $I_{0}$, corresponding to the number of people that are recently infected and infectious $\left(I^{A}\right)$ in each type $\theta$ at $t=0$.

We allow for the change in $\beta_{m t}$ from $\beta_{m}^{1}$ to $\beta_{m}^{2}$ to capture how a combination of endogenous behavioral changes and regulatory guidelines reduce contagion given contact during a pandemic. Typical examples include the use of masks, hand sanitizer, six feet distancing, etc.

We run a grid search for $\beta_{m}^{1}, \beta_{m}^{2}$, and $I_{0}$, which minimizes the sum of squared error of log death per 100 thousands people in the first 60 days of data. Then, we use $t \in(61,90)$ to test out-of-sample fit. We drop a few days after the first death or the start of the sample to avoid noise in reported deaths in the early period. Because the period in Phase 1 with reported death is short, in this calibration exercise, we consider $\beta_{m t}=\beta_{m}^{1}$ for $t \leq 25$ for NYC and Chicago, and $\beta_{m t}=\beta_{m}^{2}$ for $t \leq 23$ for Sacramento. The exact timing for this change in transmission rate $\beta_{m t}$ is selected out of a range from 20 to 30 that generates reasonable out-of-sample fit and sensible parameter values. The disease spread earlier in Sacramento, so an earlier change of behavior is more reasonable. In addition, the death rate in Sacramento is low and noisy, so we fix $I_{0}=1$ and only search for $\beta_{m}^{1}$ and $\beta_{m}^{2}$.

Table B.1: Estimated Transmission Rate $\beta_{m t}$

\begin{tabular}{lccc}
\hline City & Contact Matrix & $\beta_{m}^{1}$ & $\beta_{m}^{2}$ \\
\hline Chicago & Replica & 0.00335 & 0.00155 \\
Sacramento & Replica & 0.00133 & 0.00040 \\
NYC & FRED & 0.01859 & 0.00965 \\
\hline
\end{tabular}

Note: The table shows estimated transmission rate $\beta_{m}^{1}$ and $\beta_{m}^{2}$ in each city. $\beta_{m}^{1}$ is applied to Phase 1 from $t=0$ to $t=15$, and $\beta_{m}^{2}$ is applied to Phases 2 and 3 for $t>15$

\footnotetext{
${ }^{28}$ Website: https://github.com/CSSEGISandData/COVID-19
} 
Figure B.1: Comparing Model Simulated and Actual Daily Deaths

(a) Chicago

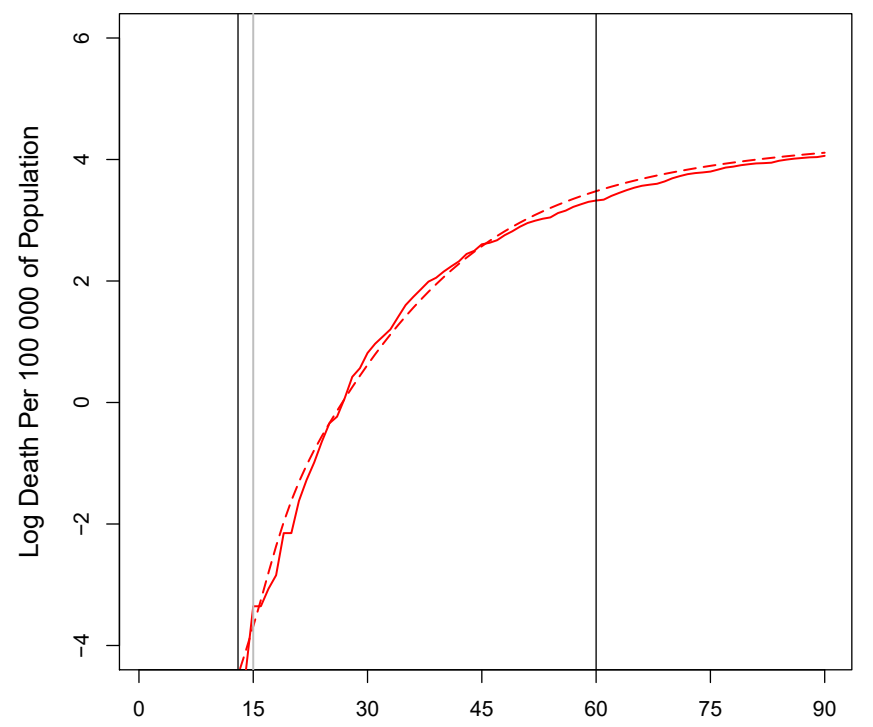

(b) Sacramento

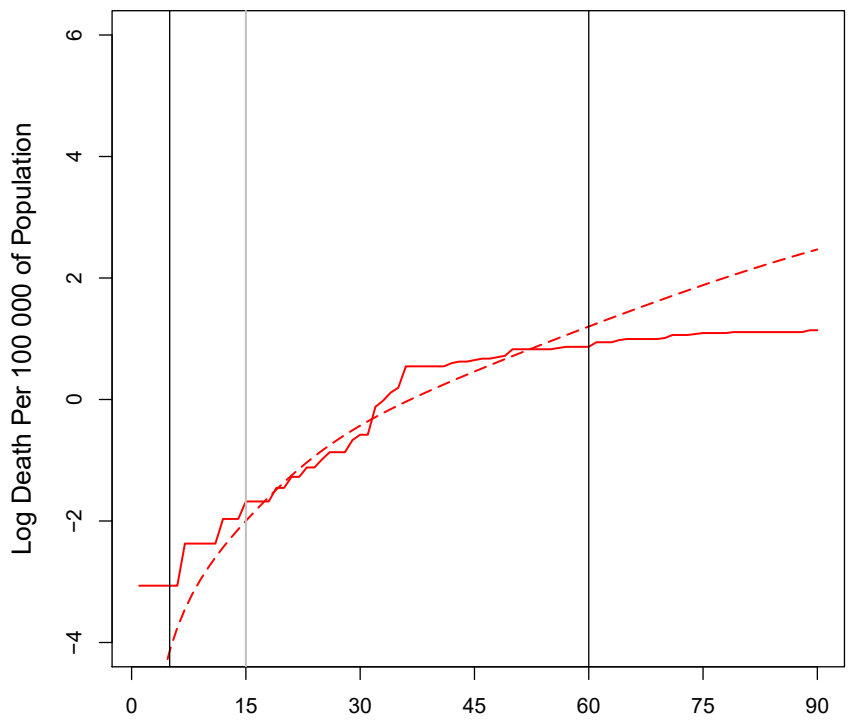

(c) NYC

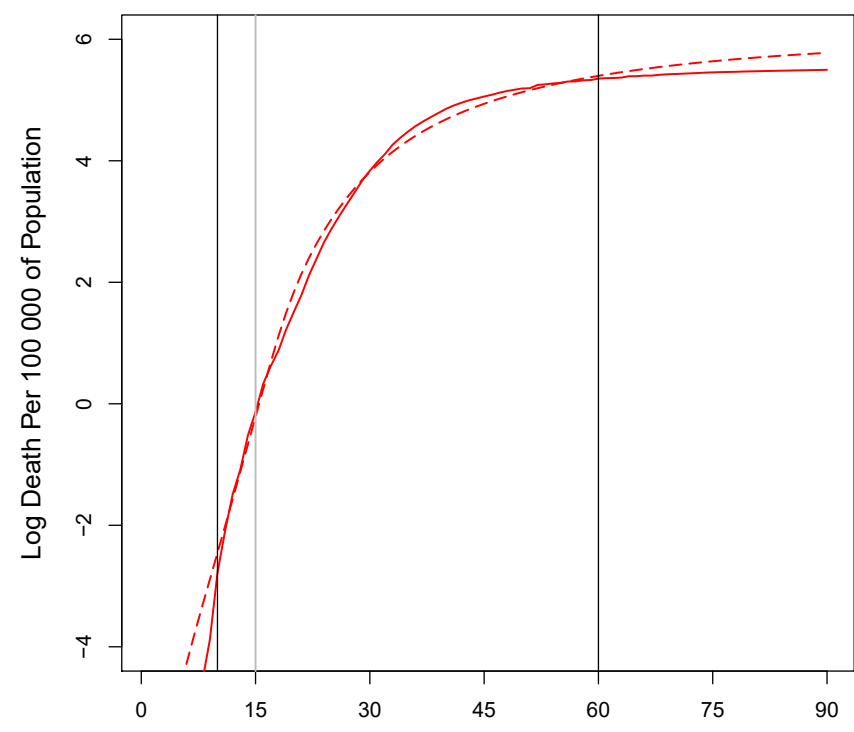

Note: Panels show results of the calibration exercise. The red solid line corresponds to the log of deaths per 100 thousand people from Johns Hopkins CSSE. The red dashed line is simulated from our model. The vertical grey line indicates the start of lock down at $t=15$, when we switch to the Essential Only (EO) contact matrix in our model. The two black vertical lines correspond to the start and end of the sample used for calibration. We minimize sum of squared errors. 


\section{Counterfactual Output}

We list tabular and graphical outcomes predicted by our empirical framework for all combinations of policies in Phase 2 and Phase 3. In terms of aggregate, cumulative outcomes we have the following results for Chicago, Sacramento, and New York, respectively. Tables are followed by a series of figures, where dashed lines will refer to the baseline case NP-EO-CR.

Table C.1: All Counterfactual Outcomes for Chicago (Replica-based)

\begin{tabular}{|c|c|c|c|c|c|c|}
\hline & Policy Sequen & & & Cumı & lative Outcom & \\
\hline Phase 1 & Phase 2 & Phase 3 & $\begin{array}{l}\text { Deaths } \\
\text { (total) }\end{array}$ & $\begin{array}{c}\text { Hospital } \\
\text { (total days) }\end{array}$ & $\begin{array}{c}\text { ICU } \\
\text { (total days) }\end{array}$ & $\begin{array}{l}\text { Employment Loss } \\
\text { (1000s days })\end{array}$ \\
\hline No Policy & No Policy & No Policy & 41251 & 1239783 & 209506 & 45577 \\
\hline No Policy & Essential Only & No Policy & 26890 & 1014953 & 165319 & 79193 \\
\hline No Policy & Alternate schedule & No Policy & 26834 & 994122 & 162417 & 56229 \\
\hline No Policy & Work from home & No Policy & 27717 & 1013997 & 166966 & 34187 \\
\hline No Policy & Isolate $60+$ & No Policy & 26449 & 961727 & 159531 & 39161 \\
\hline No Policy & Cautious Reopening & No Policy & 26590 & 952521 & 158317 & 36115 \\
\hline No Policy & No Policy & Essential Only & 36104 & 1053941 & 171167 & 95279 \\
\hline No Policy & Essential Only & Essential Only & 3693 & 120707 & 20216 & 115765 \\
\hline No Policy & Alternate schedule & Essential Only & 5220 & 176991 & 29685 & 96264 \\
\hline No Policy & Work from home & Essential Only & 5302 & 180315 & 30776 & 72743 \\
\hline No Policy & Isolate $60+$ & Essential Only & 6456 & 253201 & 43330 & 82615 \\
\hline No Policy & Cautious Reopening & Essential Only & 8259 & 284919 & 48682 & 80521 \\
\hline No Policy & No Policy & Alternate schedule & 36220 & 1062256 & 173922 & 69349 \\
\hline No Policy & Essential Only & Alternate schedule & 4328 & 158005 & 26538 & 87822 \\
\hline No Policy & Alternate schedule & Alternate schedule & 5580 & 193112 & 32509 & 67873 \\
\hline No Policy & Work from home & Alternate schedule & 5828 & 207967 & 35652 & 45082 \\
\hline No Policy & Isolate $60+$ & Alternate schedule & 6913 & 267703 & 46117 & 55049 \\
\hline No Policy & Cautious Reopening & Alternate schedule & 8478 & 295360 & 50816 & 53083 \\
\hline No Policy & No Policy & Work from home & 36312 & 1086407 & 184955 & 43485 \\
\hline No Policy & Essential Only & Work from home & 4595 & 168852 & 29224 & 59129 \\
\hline No Policy & Alternate schedule & Work from home & 5891 & 212195 & 37056 & 39748 \\
\hline No Policy & Work from home & Work from home & 5898 & 215612 & 39364 & 16199 \\
\hline No Policy & Isolate $60+$ & Work from home & 7129 & 287544 & 52693 & 27621 \\
\hline No Policy & Cautious Reopening & Work from home & 8676 & 314921 & 57500 & 25852 \\
\hline No Policy & No Policy & Isolate $60+$ & 36280 & 1090019 & 185590 & 47946 \\
\hline No Policy & Essential Only & Isolate $60+$ & 5331 & 236176 & 40870 & 69828 \\
\hline No Policy & Alternate schedule & Isolate $60+$ & 6335 & 256044 & 44628 & 48031 \\
\hline No Policy & Work from home & Isolate $60+$ & 6662 & 276572 & 49821 & 26290 \\
\hline No Policy & Isolate $60+$ & Isolate $60+$ & 7038 & 301088 & 55082 & 33644 \\
\hline No Policy & Cautious Reopening & Isolate $60+$ & 8794 & 330616 & 60234 & 31577 \\
\hline No Policy & No Policy & Cautious Reopening & 36516 & 1094672 & 186387 & 44120 \\
\hline No Policy & Essential Only & Cautious Reopening & 6765 & 267024 & 46099 & 66728 \\
\hline No Policy & Alternate schedule & Cautious Reopening & 7355 & 279298 & 48572 & 44648 \\
\hline No Policy & Work from home & Cautious Reopening & 7817 & 300993 & 53961 & 22995 \\
\hline No Policy & Isolate $60+$ & Cautious Reopening & 8349 & 325932 & 59274 & 30162 \\
\hline No Policy & Cautious Reopening & Cautious Reopening & 9271 & 341811 & 62146 & 27796 \\
\hline
\end{tabular}


Table C.2: All Counterfactual Outcomes for Sacramento (Replica-based)

\begin{tabular}{|c|c|c|c|c|c|c|}
\hline \multicolumn{3}{|c|}{ Policy Sequence } & \multicolumn{4}{|c|}{ Cumulative Outcomes } \\
\hline Phase 1 & Phase 2 & Phase 3 & $\begin{array}{l}\text { Deaths } \\
\text { (total) }\end{array}$ & $\begin{array}{c}\text { Hospital } \\
\text { (total days) }\end{array}$ & $\begin{array}{c}\text { ICU } \\
\text { (total days) }\end{array}$ & $\begin{array}{l}\text { Employment Loss } \\
\text { (1000s days) }\end{array}$ \\
\hline No Policy & No Policy & No Policy & 1274 & 46867 & 7460 & 2010 \\
\hline No Policy & Essential Only & No Policy & 902 & 36782 & 5899 & 13840 \\
\hline No Policy & Alternate schedule & No Policy & 908 & 36945 & 5925 & 7673 \\
\hline No Policy & Work from home & No Policy & 914 & 37127 & 5956 & 1506 \\
\hline No Policy & Isolate $60+$ & No Policy & 922 & 37380 & 5996 & 2345 \\
\hline No Policy & Cautious Reopening & No Policy & 928 & 37517 & 6018 & 1542 \\
\hline No Policy & No Policy & Essential Only & 636 & 22424 & 3707 & 16998 \\
\hline No Policy & Essential Only & Essential Only & 398 & 15923 & 2691 & 28986 \\
\hline No Policy & Alternate schedule & Essential Only & 400 & 16002 & 2705 & 22814 \\
\hline No Policy & Work from home & Essential Only & 403 & 16088 & 2721 & 16642 \\
\hline No Policy & Isolate $60+$ & Essential Only & 406 & 16202 & 2740 & 17474 \\
\hline No Policy & Cautious Reopening & Essential Only & 410 & 16277 & 2753 & 16667 \\
\hline No Policy & No Policy & Alternate schedule & 641 & 22687 & 3753 & 9216 \\
\hline No Policy & Essential Only & Alternate schedule & 401 & 16088 & 2720 & 21167 \\
\hline No Policy & Alternate schedule & Alternate schedule & 403 & 16169 & 2734 & 14996 \\
\hline No Policy & Work from home & Alternate schedule & 406 & 16257 & 2750 & 8826 \\
\hline No Policy & Isolate $60+$ & Alternate schedule & 409 & 16375 & 2770 & 9659 \\
\hline No Policy & Cautious Reopening & Alternate schedule & 413 & 16452 & 2783 & 8853 \\
\hline No Policy & No Policy & Work from home & 646 & 22961 & 3806 & 1445 \\
\hline No Policy & Essential Only & Work from home & 404 & 16302 & 2760 & 13356 \\
\hline No Policy & Alternate schedule & Work from home & 407 & 16388 & 2775 & 7187 \\
\hline No Policy & Work from home & Work from home & 410 & 16482 & 2792 & 1018 \\
\hline No Policy & Isolate $60+$ & Work from home & 413 & 16594 & 2811 & 1853 \\
\hline No Policy & Cautious Reopening & Work from home & 417 & 16671 & 2824 & 1048 \\
\hline No Policy & No Policy & Isolate $60+$ & 648 & 23358 & 3872 & 2514 \\
\hline No Policy & Essential Only & Isolate $60+$ & 405 & 16518 & 2796 & 14409 \\
\hline No Policy & Alternate schedule & Isolate $60+$ & 408 & 16605 & 2811 & 8239 \\
\hline No Policy & Work from home & Isolate $60+$ & 411 & 16699 & 2828 & 2071 \\
\hline No Policy & Isolate $60+$ & Isolate $60+$ & 414 & 16829 & 2850 & 2908 \\
\hline No Policy & Cautious Reopening & Isolate $60+$ & 418 & 16910 & 2864 & 2103 \\
\hline No Policy & No Policy & Cautious Reopening & 661 & 23675 & 3926 & 1505 \\
\hline No Policy & Essential Only & Cautious Reopening & 411 & 16687 & 2825 & 13386 \\
\hline No Policy & Alternate schedule & Cautious Reopening & 414 & 16776 & 2840 & 7217 \\
\hline No Policy & Work from home & Cautious Reopening & 417 & 16873 & 2858 & 1049 \\
\hline No Policy & Isolate $60+$ & Cautious Reopening & 420 & 17007 & 2880 & 1885 \\
\hline No Policy & Cautious Reopening & Cautious Reopening & 424 & 17091 & 2895 & 1081 \\
\hline
\end{tabular}


Table C.3: All Counterfactual Outcomes for New York (FRED-based)

\begin{tabular}{|c|c|c|c|c|c|c|}
\hline \multicolumn{3}{|c|}{ Policy Sequence } & \multicolumn{4}{|c|}{ Cumulative Outcomes } \\
\hline Phase 1 & Phase 2 & Phase 3 & $\begin{array}{l}\text { Deaths } \\
\text { (total) }\end{array}$ & $\begin{array}{c}\text { Hospital } \\
\text { (total days) }\end{array}$ & $\begin{array}{c}\text { ICU } \\
\text { (total days) }\end{array}$ & $\begin{array}{c}\text { Employment Loss } \\
\text { (1000s days })\end{array}$ \\
\hline No Policy & No Policy & No Policy & 75580 & 2412392 & 449056 & 92641 \\
\hline No Policy & Essential Only & No Policy & 70507 & 2384497 & 444773 & 174636 \\
\hline No Policy & Alternate schedule & No Policy & 70180 & 2367081 & 442112 & 130080 \\
\hline No Policy & Work from home & No Policy & 69378 & 2343724 & 438878 & 88581 \\
\hline No Policy & Isolate $60+$ & No Policy & 67554 & 2300829 & 432506 & 97260 \\
\hline No Policy & Cautious Reopening & No Policy & 67617 & 2292707 & 431381 & 90802 \\
\hline No Policy & No Policy & Essential Only & 75516 & 2410579 & 448787 & 207158 \\
\hline No Policy & Essential Only & Essential Only & 30547 & 1067264 & 202093 & 258112 \\
\hline No Policy & Alternate schedule & Essential Only & 36357 & 1287956 & 249352 & 222854 \\
\hline No Policy & Work from home & Essential Only & 39147 & 1437947 & 296731 & 182785 \\
\hline No Policy & Isolate $60+$ & Essential Only & 40947 & 1550985 & 315685 & 201779 \\
\hline No Policy & Cautious Reopening & Essential Only & 44154 & 1600061 & 323985 & 197515 \\
\hline No Policy & No Policy & Alternate schedule & 75516 & 2410588 & 448790 & 149896 \\
\hline No Policy & Essential Only & Alternate schedule & 32287 & 1162454 & 223004 & 203634 \\
\hline No Policy & Alternate schedule & Alternate schedule & 37358 & 1341988 & 262246 & 168547 \\
\hline No Policy & Work from home & Alternate schedule & 40030 & 1478616 & 304417 & 128869 \\
\hline No Policy & Isolate $60+$ & Alternate schedule & 41404 & 1564842 & 318830 & 146784 \\
\hline No Policy & Cautious Reopening & Alternate schedule & 44296 & 1608217 & 326150 & 142437 \\
\hline No Policy & No Policy & Work from home & 75516 & 2410603 & 448796 & 92634 \\
\hline No Policy & Essential Only & Work from home & 34057 & 1301209 & 265798 & 149612 \\
\hline No Policy & Alternate schedule & Work from home & 38471 & 1429428 & 290151 & 114563 \\
\hline No Policy & Work from home & Work from home & 39848 & 1476895 & 306748 & 72612 \\
\hline No Policy & Isolate $60+$ & Work from home & 41685 & 1581897 & 323960 & 91878 \\
\hline No Policy & Cautious Reopening & Work from home & 44496 & 1623061 & 330857 & 87471 \\
\hline No Policy & No Policy & Isolate $60+$ & 75516 & 2410609 & 448797 & 102091 \\
\hline No Policy & Essential Only & Isolate $60+$ & 35824 & 1426563 & 287545 & 169914 \\
\hline No Policy & Alternate schedule & Isolate $60+$ & 39133 & 1485593 & 299698 & 128990 \\
\hline No Policy & Work from home & Isolate $60+$ & 41121 & 1557386 & 319959 & 88835 \\
\hline No Policy & Isolate $60+$ & Isolate $60+$ & 41399 & 1582603 & 324062 & 101806 \\
\hline No Policy & Cautious Reopening & Isolate $60+$ & 44528 & 1628035 & 331679 & 97191 \\
\hline No Policy & No Policy & Cautious Reopening & 75517 & 2410615 & 448798 & 92635 \\
\hline No Policy & Essential Only & Cautious Reopening & 38431 & 1476815 & 296186 & 163036 \\
\hline No Policy & Alternate schedule & Cautious Reopening & 40530 & 1513626 & 304477 & 121170 \\
\hline No Policy & Work from home & Cautious Reopening & 42473 & 1583275 & 324289 & 80961 \\
\hline No Policy & Isolate $60+$ & Cautious Reopening & 42686 & 1604187 & 327691 & 93333 \\
\hline No Policy & Cautious Reopening & Cautious Reopening & 44706 & 1631531 & 332265 & 88207 \\
\hline
\end{tabular}


Figure C.1: NP-EO-CR Policy Sequence in Chicago (Replica Based)

(a) Chicago, Epidemic Outcomes

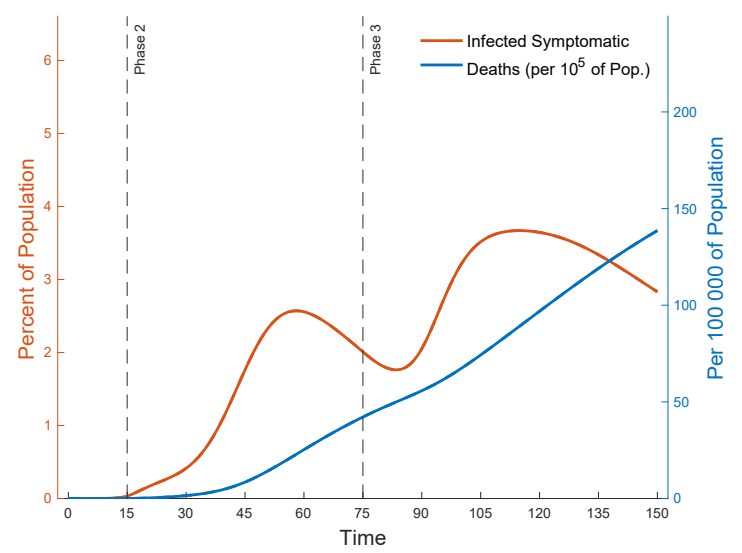

(b) Chicago, Employment Outcomes

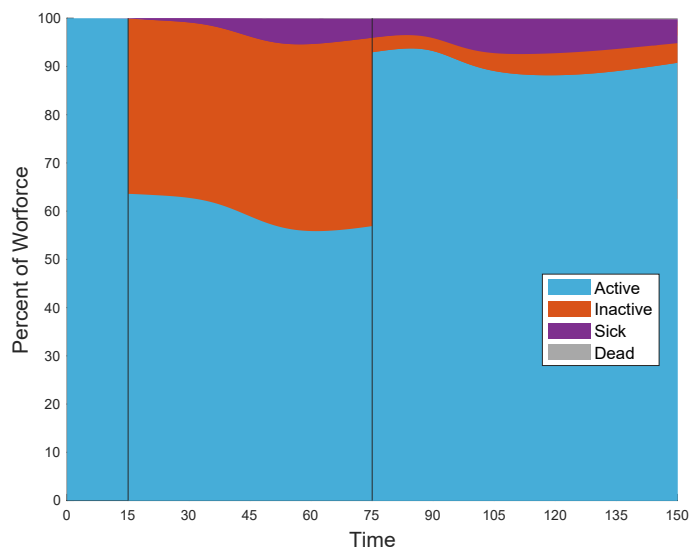

(c) Chicago, Healthcare Outcomes

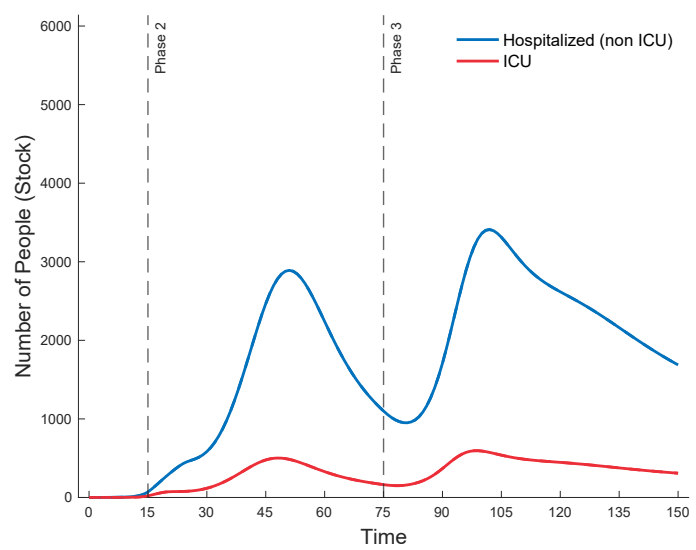

Note: The figure shows health and employment outcomes with No Policy in Phase 1, Essential Only in Phase 2 and Cautious Reopening in Phase 3 for the Chicago MSA, with contact matrices based on Replica data. The top left panel displays the percent of individuals of the local population that are infected and symptomatic and/or detected, and the number of deaths per 100,000s of population on the right vertical axis. The top right panel corresponds plots the share of workforce that is either active, inactive (due to quarantine, or not allowed to access the workplace and unable to work from home), sick, or deceased. Dashed lines correspond to the baseline case NP-EO-CR. 
Figure C.2: NP-EO-EO Policy Sequence in Chicago (Replica Based)

(a) Chicago, Epidemic Outcomes

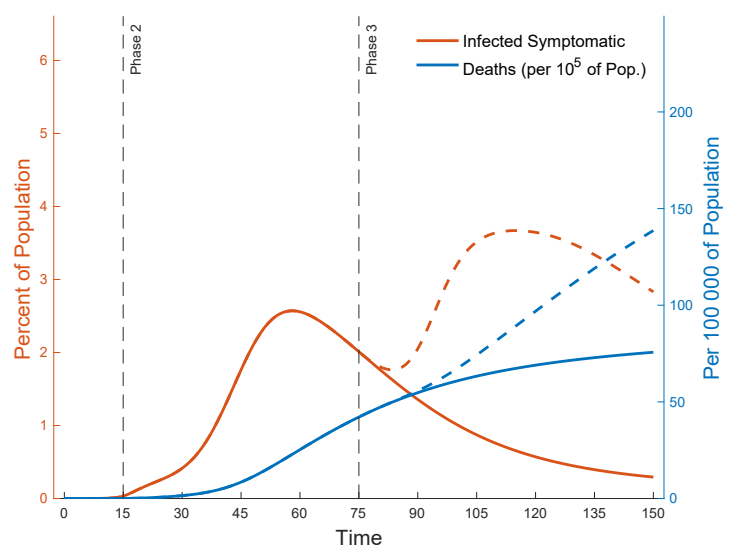

(b) Chicago, Employment Outcomes

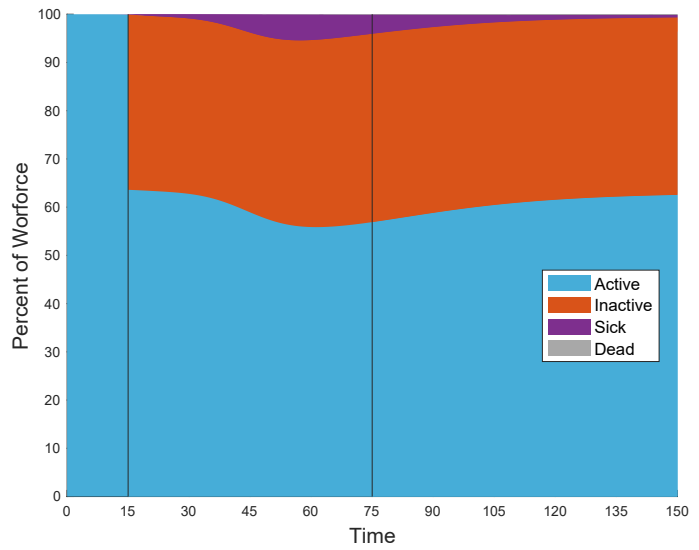

(c) Chicago, Healthcare Outcomes

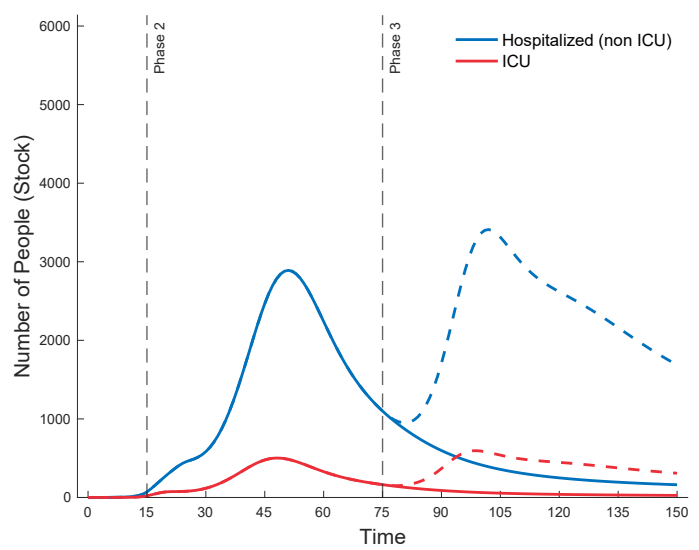

Note: The figure shows health and employment outcomes with No Policy in Phase 1, Essential Only in Phase 2 and Essential Only in Phase 3 for the Chicago MSA, with contact matrices based on Replica data. The top left panel displays the percent of individuals of the local population that are infected and symptomatic and/or detected, and the number of deaths per 100,000s of population on the right vertical axis. The top right panel corresponds plots the share of workforce that is either active, inactive (due to quarantine, or not allowed to access the workplace and unable to work from home), sick, or deceased. Dashed lines correspond to the baseline case NP-EO-CR. 
Figure C.3: NP-EO-60+ Policy Sequence in Chicago (Replica Based)

(a) Chicago, Epidemic Outcomes

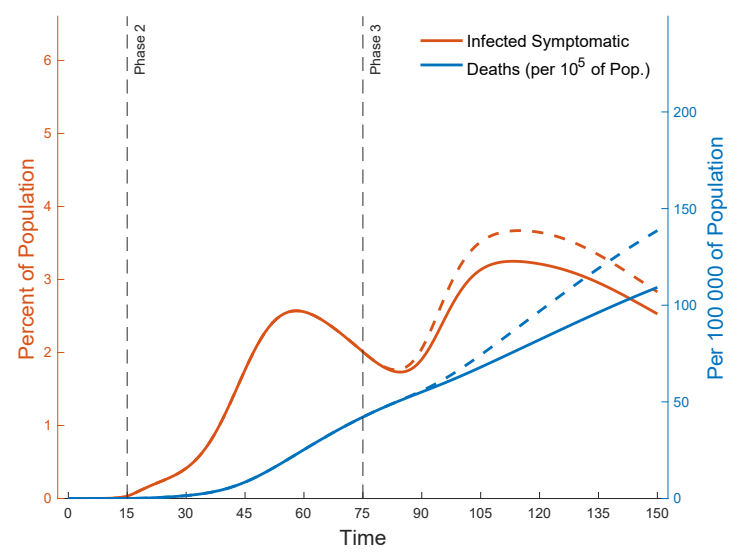

(b) Chicago, Employment Outcomes

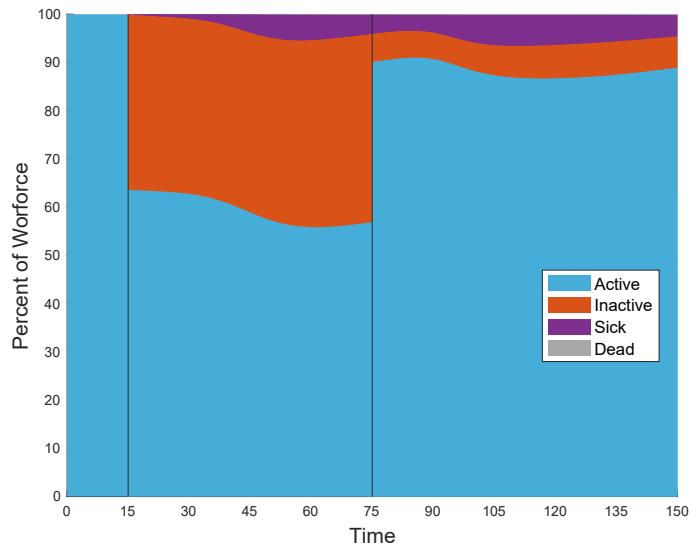

(c) Chicago, Healthcare Outcomes

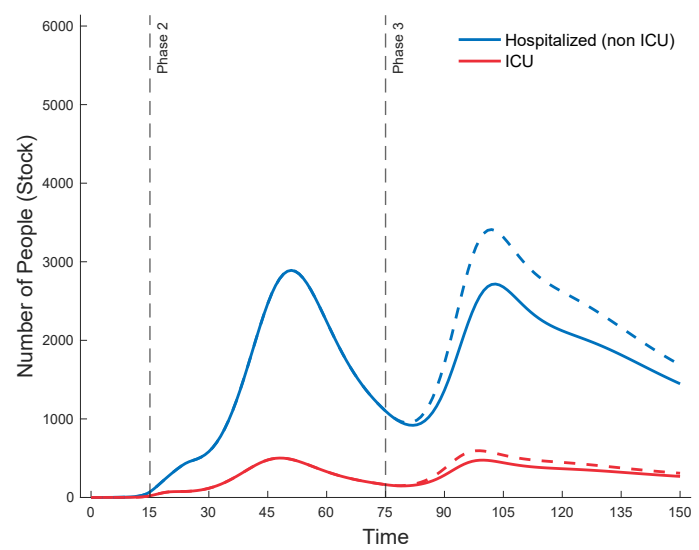

Note: The figure shows health and employment outcomes with No Policy in Phase 1, Essential Only in Phase 2 and Isolate 60+ in Phase 3 for the Chicago MSA, with contact matrices based on Replica data. The top left panel displays the percent of individuals of the local population that are infected and symptomatic and/or detected, and the number of deaths per 100,000s of population on the right vertical axis. The top right panel corresponds plots the share of workforce that is either active, inactive (due to quarantine, or not allowed to access the workplace and unable to work from home), sick, or deceased. Dashed lines correspond to the baseline case NP-EO-CR. 
Figure C.4: NP-EO-WFH Policy Sequence in Chicago (Replica Based)

(a) Chicago, Epidemic Outcomes

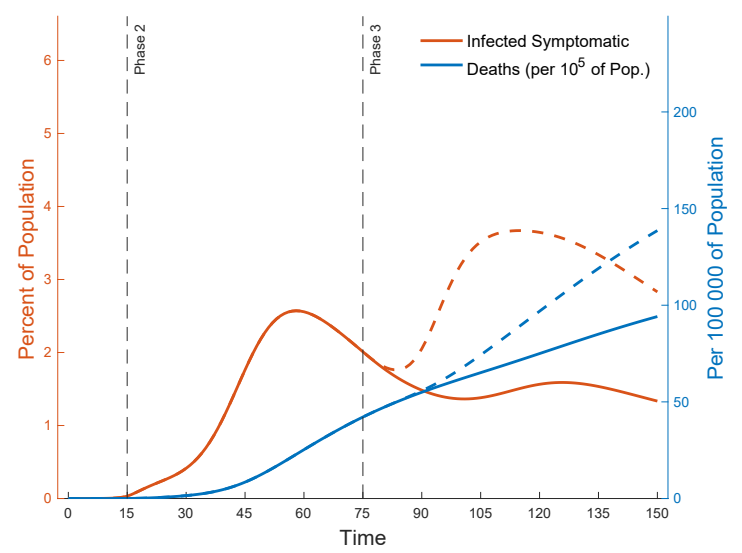

(b) Chicago, Employment Outcomes

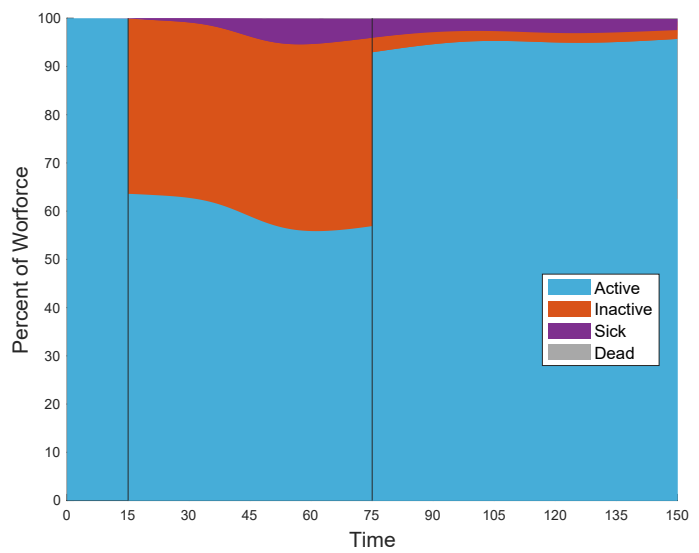

(c) Chicago, Healthcare Outcomes

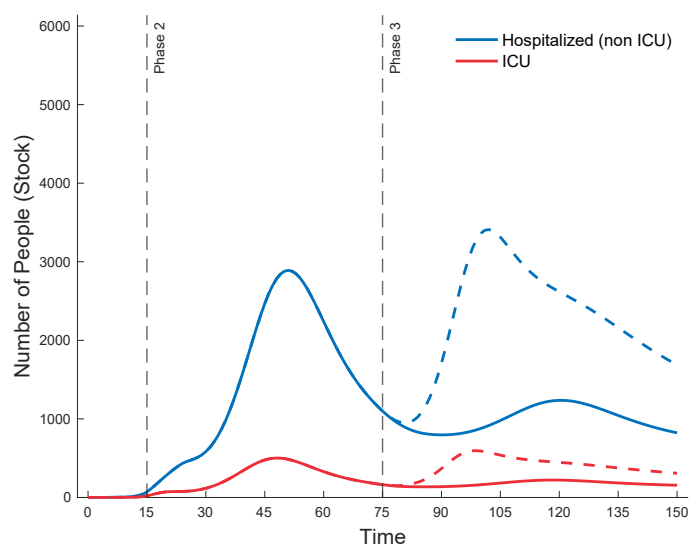

Note: The figure shows health and employment outcomes with No Policy in Phase 1, Essential Only in Phase 2 and Work-from-Home if Possible in Phase 3 for the Chicago MSA, with contact matrices based on Replica data. The top left panel displays the percent of individuals of the local population that are infected and symptomatic and/or detected, and the number of deaths per 100,000s of population on the right vertical axis. The top right panel corresponds plots the share of workforce that is either active, inactive (due to quarantine, or not allowed to access the workplace and unable to work from home), sick, or deceased. Dashed lines correspond to the baseline case NP-EO-CR. 
Figure C.5: NP-EO-AS Policy Sequence in Chicago (Replica Based)

(a) Chicago, Epidemic Outcomes

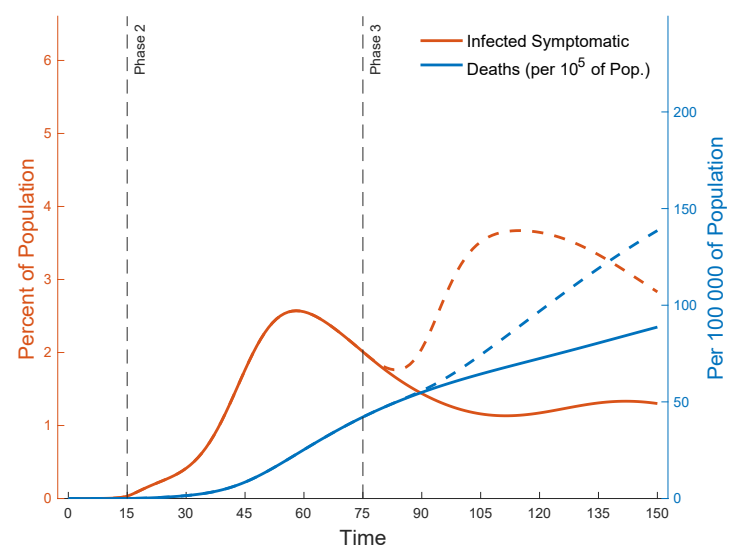

(b) Chicago, Employment Outcomes

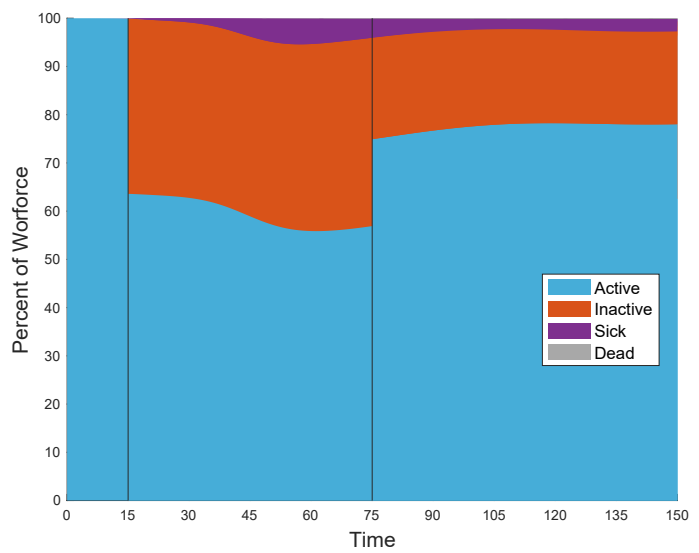

(c) Chicago, Healthcare Outcomes

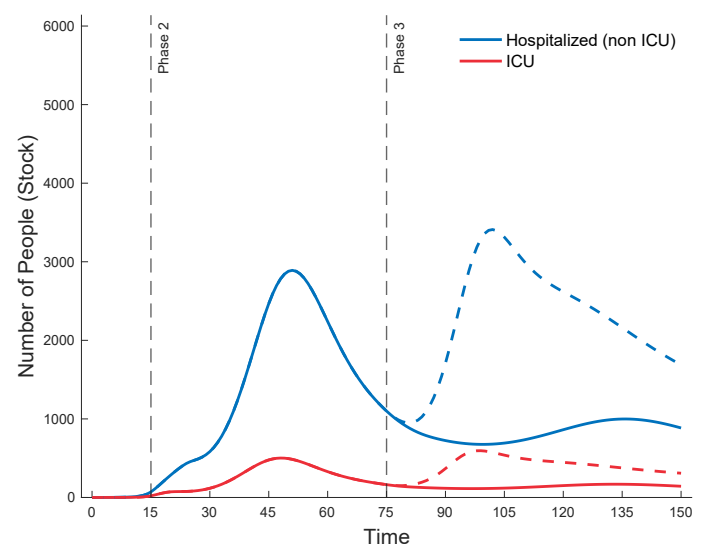

Note: The figure shows health and employment outcomes with No Policy in Phase 1, Essential Only in Phase 2 and Alternating Schedules in Phase 3 for the Chicago MSA, with contact matrices based on Replica data. The top left panel displays the percent of individuals of the local population that are infected and symptomatic and/or detected, and the number of deaths per 100,000s of population on the right vertical axis. The top right panel corresponds plots the share of workforce that is either active, inactive (due to quarantine, or not allowed to access the workplace and unable to work from home), sick, or deceased. Dashed lines correspond to the baseline case NP-EO-CR. 
Figure C.6: NP-CR-CR Policy Sequence in Chicago (Replica Based)

(a) Chicago, Epidemic Outcomes

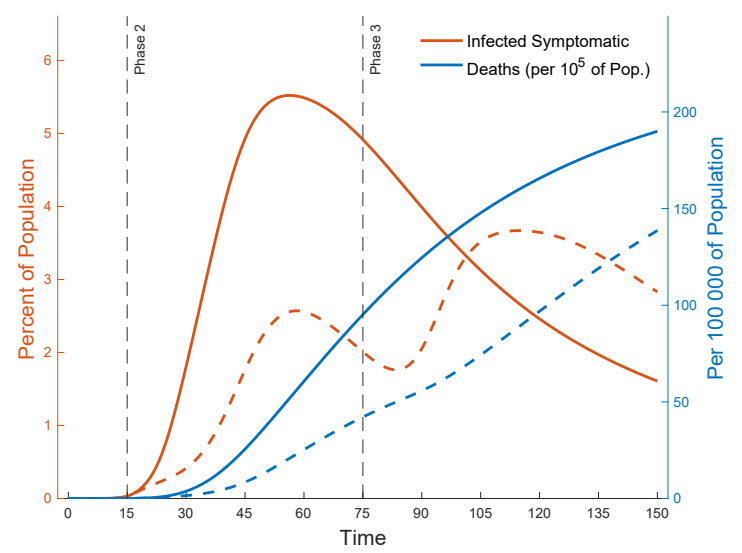

(b) Chicago, Employment Outcomes

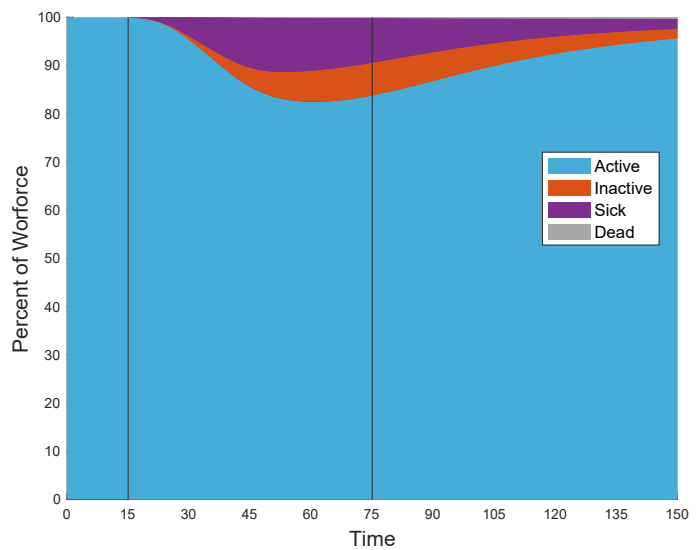

(c) Chicago, Healthcare Outcomes

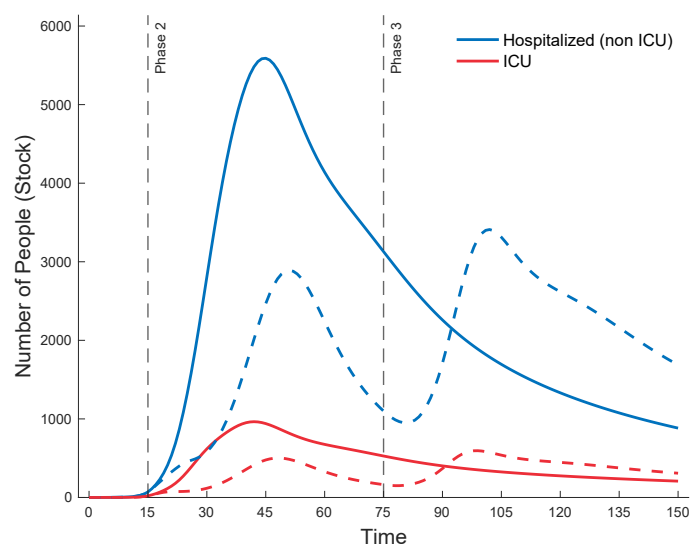

Note: The figure shows health and employment outcomes with No Policy in Phase 1, Cautious Reopening in Phase 2 and Cautious Reopening in Phase 3 for the Chicago MSA, with contact matrices based on Replica data. The top left panel displays the percent of individuals of the local population that are infected and symptomatic and/or detected, and the number of deaths per 100,000s of population on the right vertical axis. The top right panel corresponds plots the share of workforce that is either active, inactive (due to quarantine, or not allowed to access the workplace and unable to work from home), sick, or deceased. Dashed lines correspond to the baseline case NP-EO-CR. 
Figure C.7: NP-60+-60+ Policy Sequence in Chicago (Replica Based)

(a) Chicago, Epidemic Outcomes

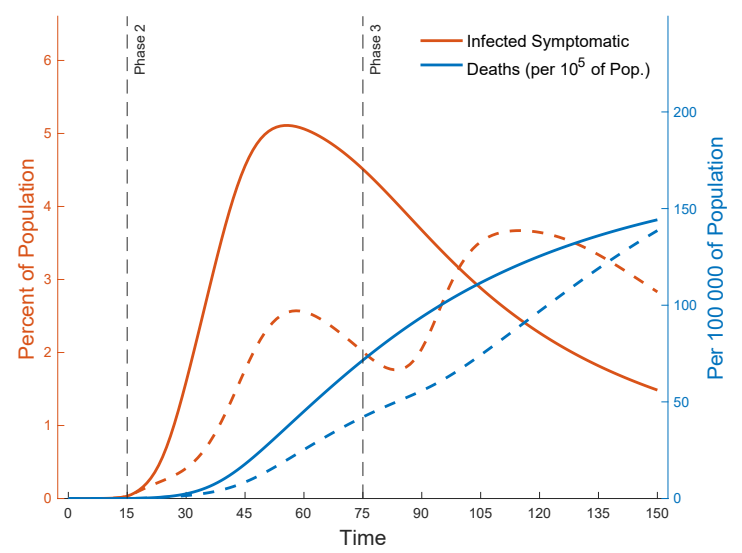

(b) Chicago, Employment Outcomes

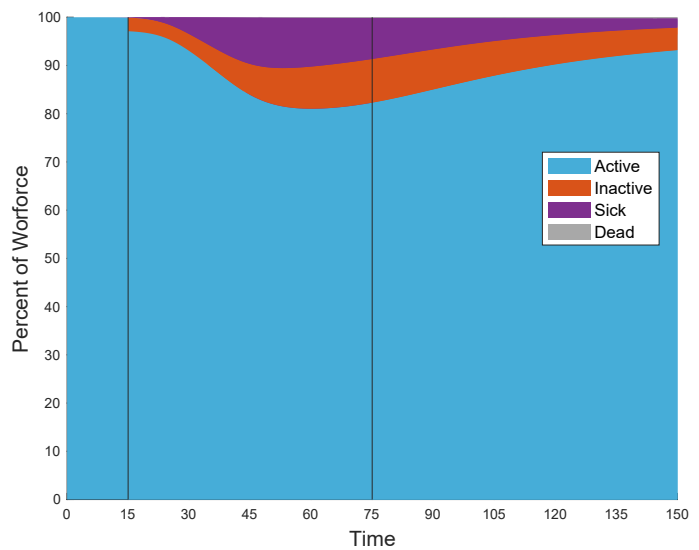

(c) Chicago, Healthcare Outcomes

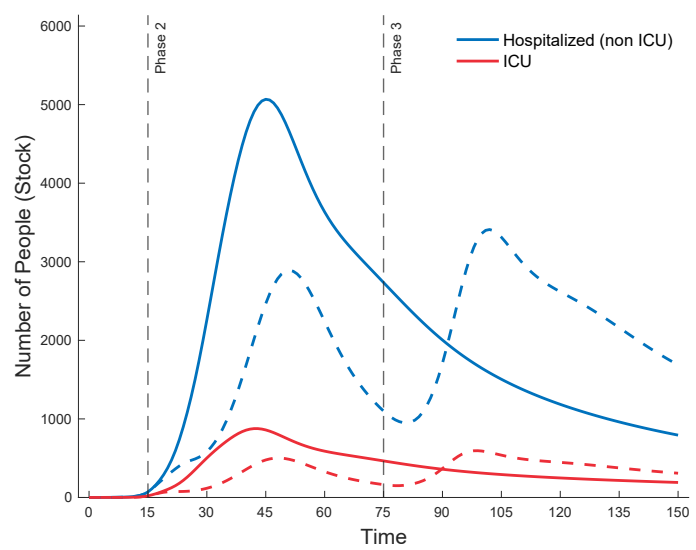

Note: The figure shows health and employment outcomes with No Policy in Phase 1, Isolate 60+ in Phase 2 and Isolate 60+ in Phase 3 for the Chicago MSA, with contact matrices based on Replica data. The top left panel displays the percent of individuals of the local population that are infected and symptomatic and/or detected, and the number of deaths per 100,000s of population on the right vertical axis. The top right panel corresponds plots the share of workforce that is either active, inactive (due to quarantine, or not allowed to access the workplace and unable to work from home), sick, or deceased. Dashed lines correspond to the baseline case NP-EO-CR. 
Figure C.8: NP-WFH-WFH Policy Sequence in Chicago (Replica Based)

(a) Chicago, Epidemic Outcomes

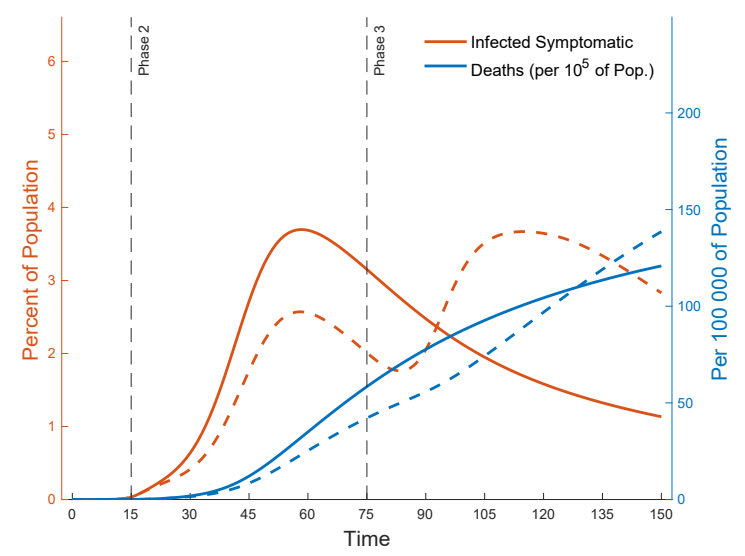

(b) Chicago, Employment Outcomes

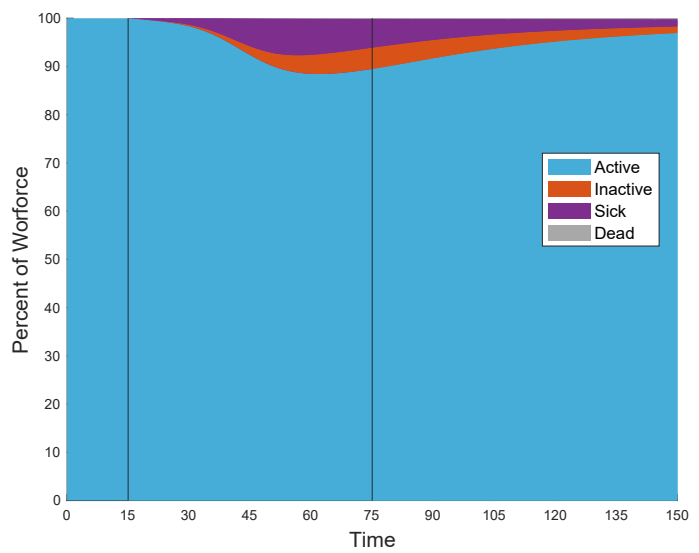

(c) Chicago, Healthcare Outcomes

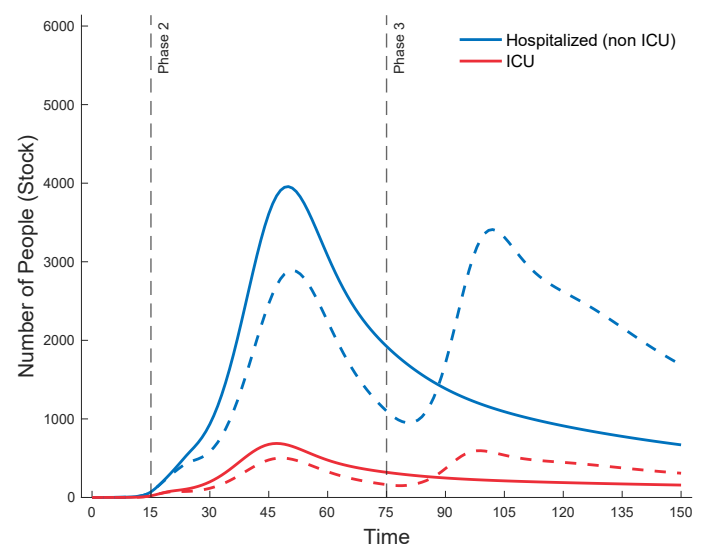

Note: The figure shows health and employment outcomes with No Policy in Phase 1, Work-from-Home if Possible in Phase 2 and Work-from-Home if Possible in Phase 3 for the Chicago MSA, with contact matrices based on Replica data. The top left panel displays the percent of individuals of the local population that are infected and symptomatic and/or detected, and the number of deaths per 100,000s of population on the right vertical axis. The top right panel corresponds plots the share of workforce that is either active, inactive (due to quarantine, or not allowed to access the workplace and unable to work from home), sick, or deceased. Dashed lines correspond to the baseline case NP-EO-CR. 


\section{Figure C.9: NP-AS-AS Policy Sequence in Chicago (Replica Based)}

(a) Chicago, Epidemic Outcomes

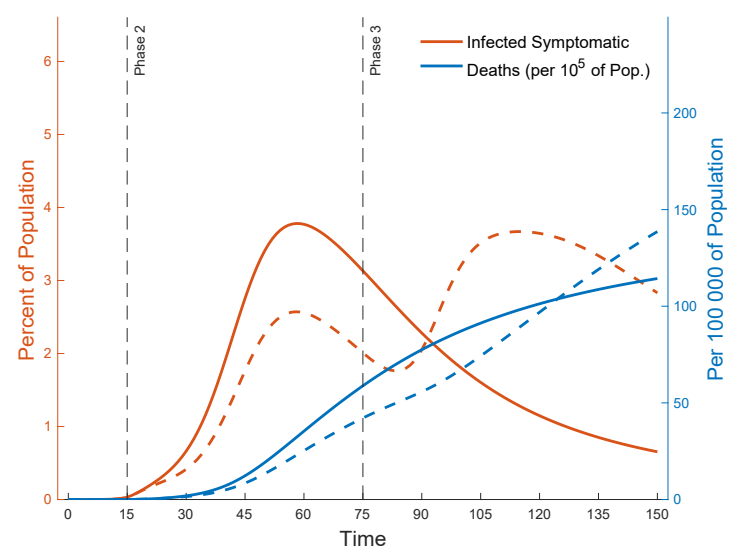

(b) Chicago, Employment Outcomes

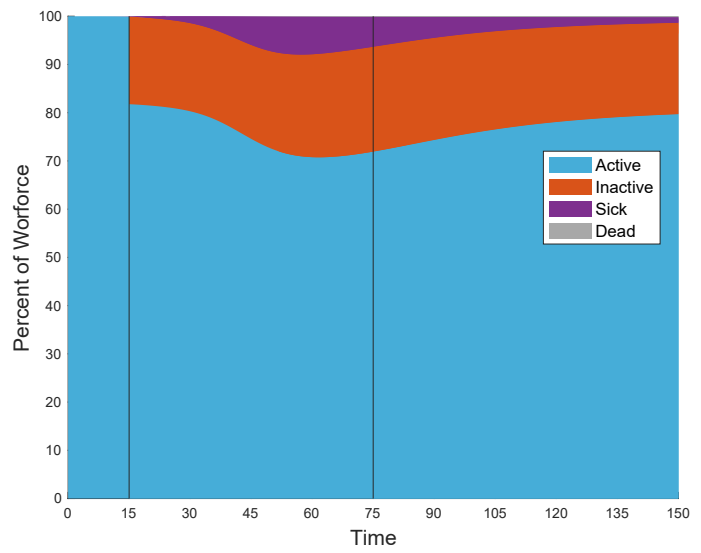

(c) Chicago, Healthcare Outcomes

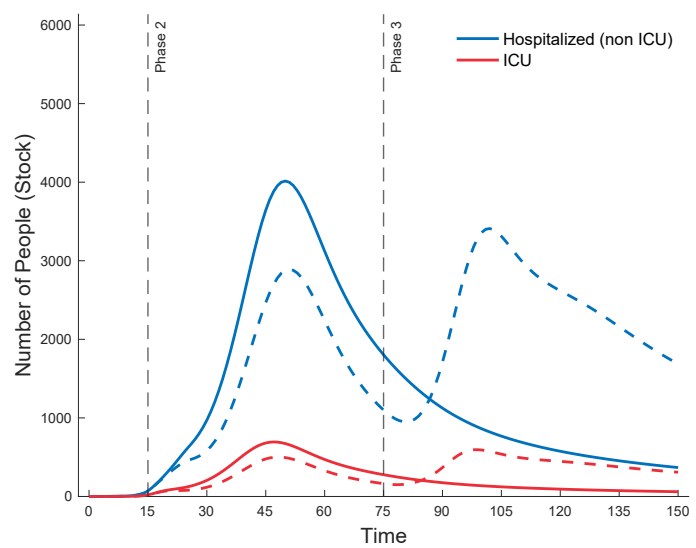

Note: The figure shows health and employment outcomes with No Policy in Phase 1, Alternating Schedules in Phase 2 and Alternating Schedules in Phase 3 for the Chicago MSA, with contact matrices based on Replica data. The top left panel displays the percent of individuals of the local population that are infected and symptomatic and/or detected, and the number of deaths per 100,000s of population on the right vertical axis. The top right panel corresponds plots the share of workforce that is either active, inactive (due to quarantine, or not allowed to access the workplace and unable to work from home), sick, or deceased. Dashed lines correspond to the baseline case NP-EO-CR. 
Figure C.10: NP-EO-CR Policy Sequence in Sacramento (Replica Based)

(a) Sacramento, Epidemic Outcomes

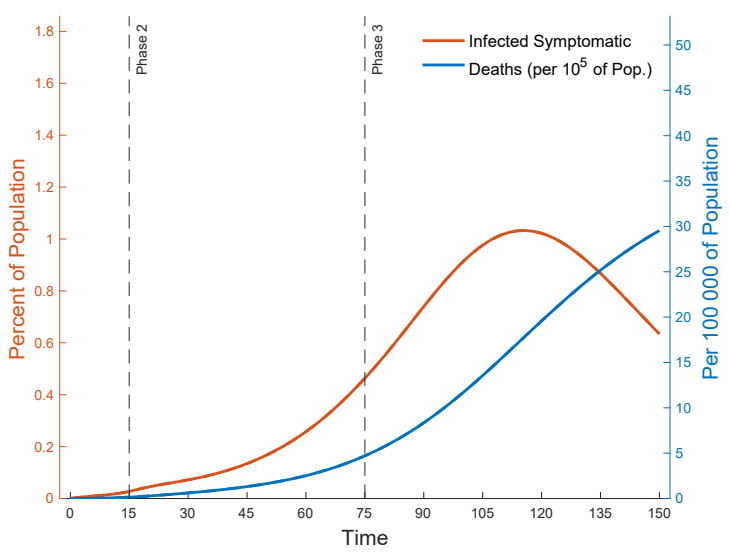

(b) Sacramento, Employment Outcomes

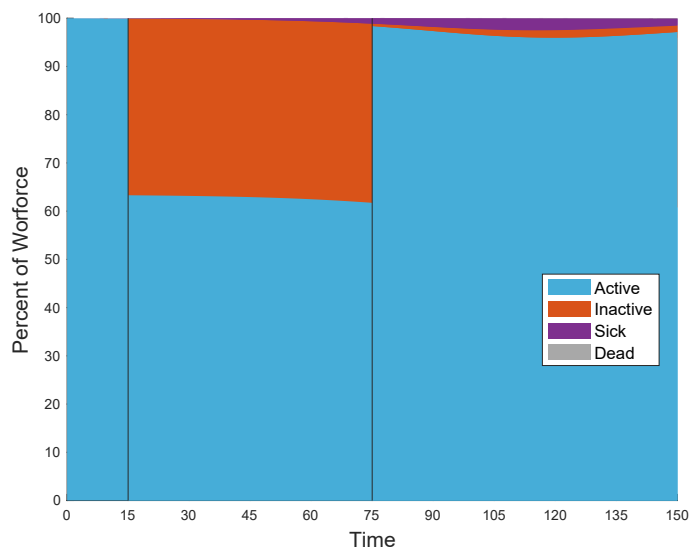

(c) Sacramento, Healthcare Outcomes

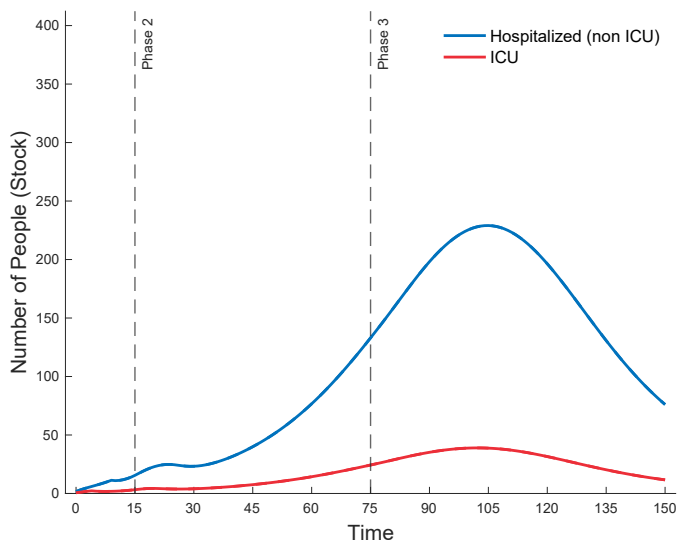

Note: The figure shows health and employment outcomes with No Policy in Phase 1, Essential Only in Phase 2 and Cautious Reopening in Phase 3 for the Sacramento MSA, with contact matrices based on Replica data. The top left panel displays the percent of individuals of the local population that are infected and symptomatic and/or detected, and the number of deaths per 100,000s of population on the right vertical axis. The top right panel corresponds plots the share of workforce that is either active, inactive (due to quarantine, or not allowed to access the workplace and unable to work from home), sick, or deceased. Dashed lines correspond to the baseline case NP-EO-CR. 
Figure C.11: NP-EO-EO Policy Sequence in Sacramento (Replica Based)

(a) Sacramento, Epidemic Outcomes

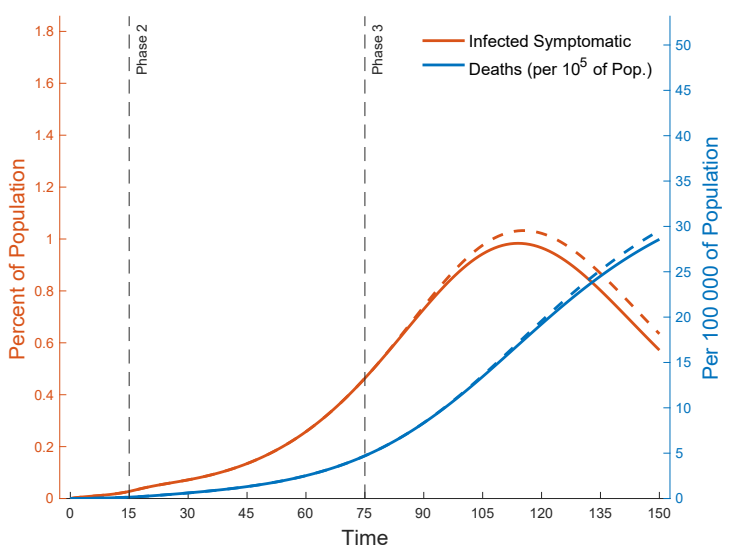

(b) Sacramento, Employment Outcomes

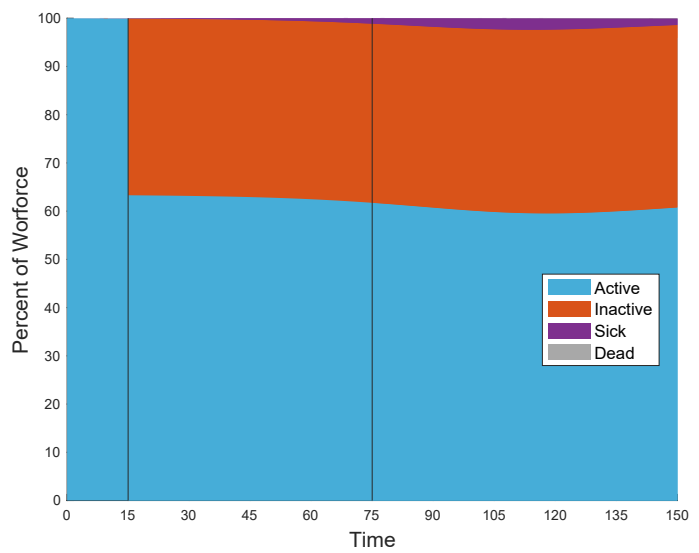

(c) Sacramento, Healthcare Outcomes

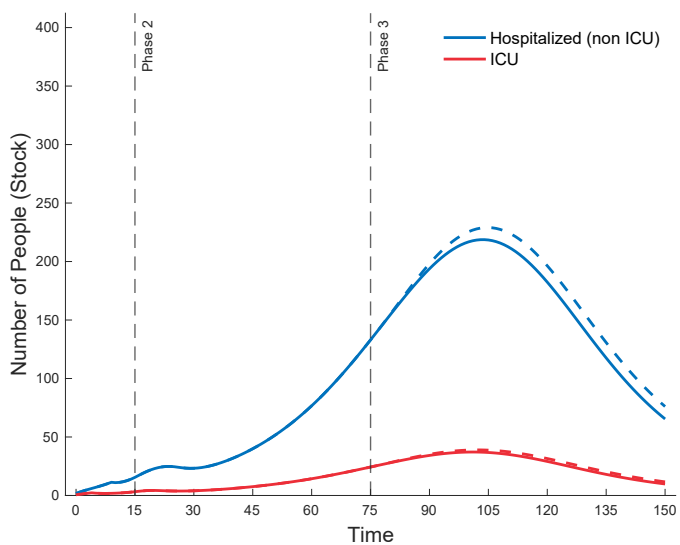

Note: The figure shows health and employment outcomes with No Policy in Phase 1, Essential Only in Phase 2 and Essential Only in Phase 3 for the Sacramento MSA, with contact matrices based on Replica data. The top left panel displays the percent of individuals of the local population that are infected and symptomatic and/or detected, and the number of deaths per 100,000s of population on the right vertical axis. The top right panel corresponds plots the share of workforce that is either active, inactive (due to quarantine, or not allowed to access the workplace and unable to work from home), sick, or deceased. Dashed lines correspond to the baseline case NP-EO-CR. 
Figure C.12: NP-EO-60+ Policy Sequence in Sacramento (Replica Based)

(a) Sacramento, Epidemic Outcomes

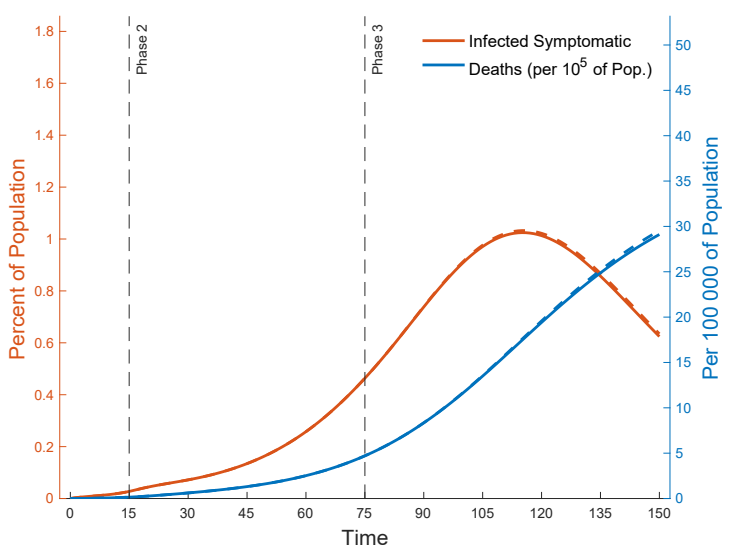

(b) Sacramento, Employment Outcomes

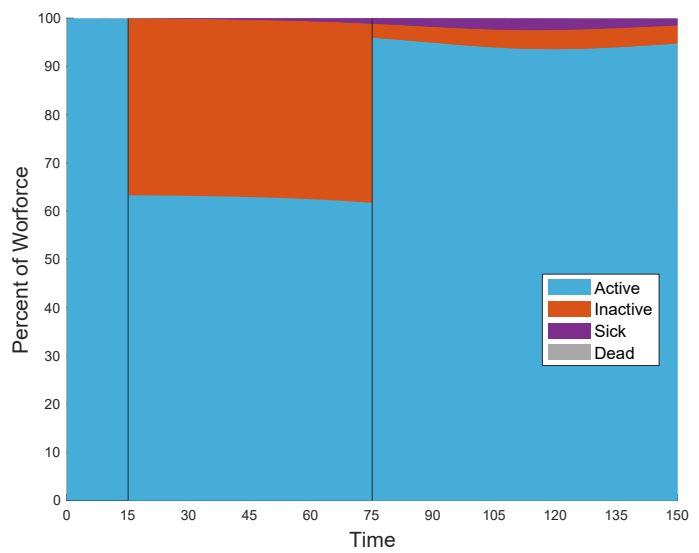

(c) Sacramento, Healthcare Outcomes

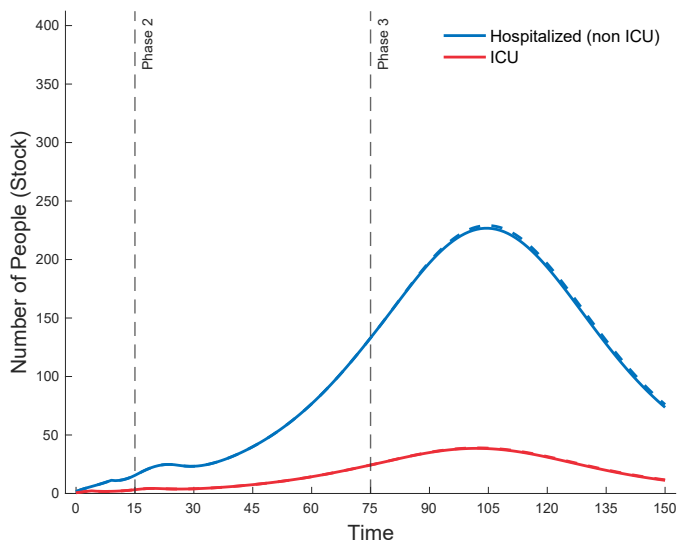

Note: The figure shows health and employment outcomes with No Policy in Phase 1, Essential Only in Phase 2 and Isolate $60+$ in Phase 3 for the Sacramento MSA, with contact matrices based on Replica data. The top left panel displays the percent of individuals of the local population that are infected and symptomatic and/or detected, and the number of deaths per 100,000s of population on the right vertical axis. The top right panel corresponds plots the share of workforce that is either active, inactive (due to quarantine, or not allowed to access the workplace and unable to work from home), sick, or deceased. Dashed lines correspond to the baseline case NP-EO-CR. 
Figure C.13: NP-EO-WFH Policy Sequence in Sacramento (Replica Based)

(a) Sacramento, Epidemic Outcomes

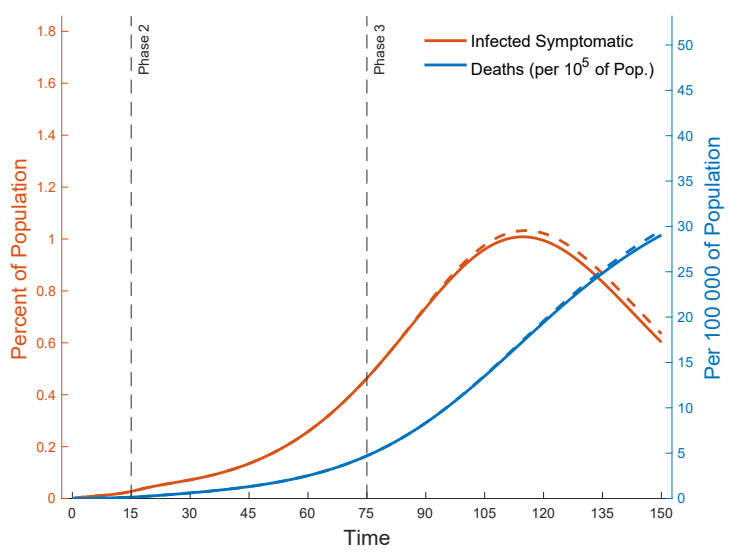

(b) Sacramento, Employment Outcomes

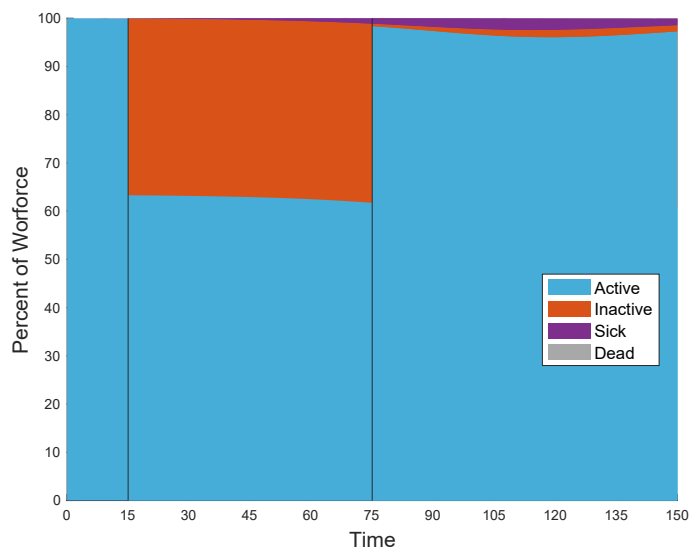

(c) Sacramento, Healthcare Outcomes

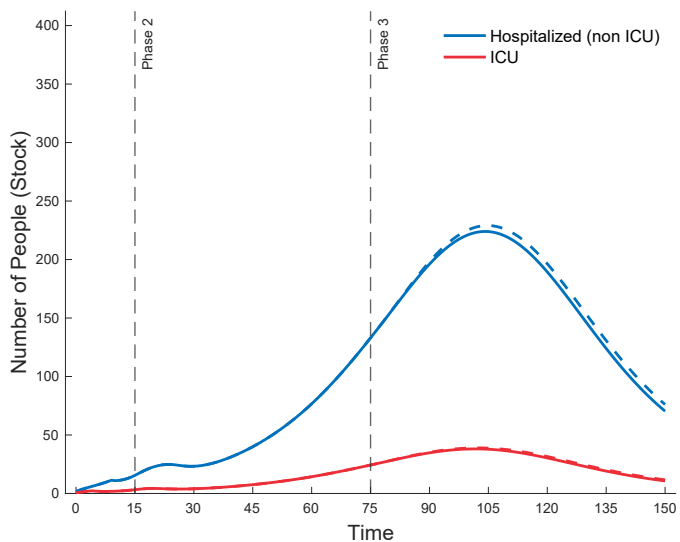

Note: The figure shows health and employment outcomes with No Policy in Phase 1, Essential Only in Phase 2 and Work-from-Home if Possible in Phase 3 for the Sacramento MSA, with contact matrices based on Replica data. The top left panel displays the percent of individuals of the local population that are infected and symptomatic and/or detected, and the number of deaths per 100,000s of population on the right vertical axis. The top right panel corresponds plots the share of workforce that is either active, inactive (due to quarantine, or not allowed to access the workplace and unable to work from home), sick, or deceased. Dashed lines correspond to the baseline case NP-EO-CR. 
Figure C.14: NP-EO-AS Policy Sequence in Sacramento (Replica Based)

(a) Sacramento, Epidemic Outcomes

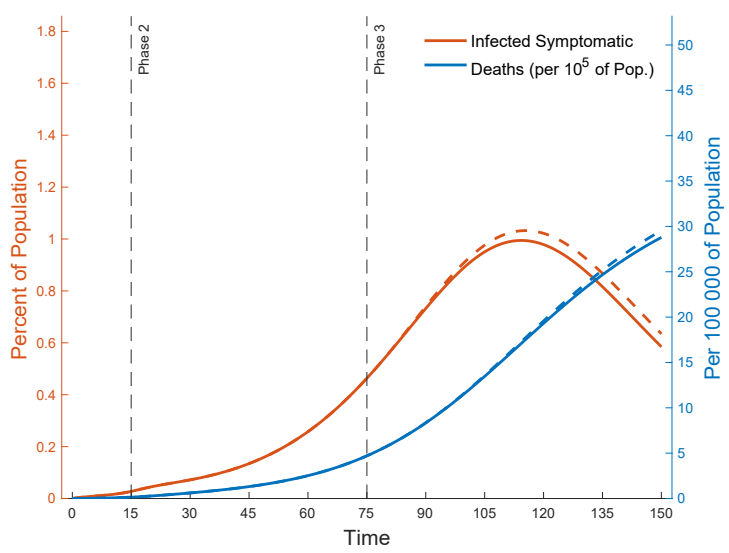

(b) Sacramento, Employment Outcomes

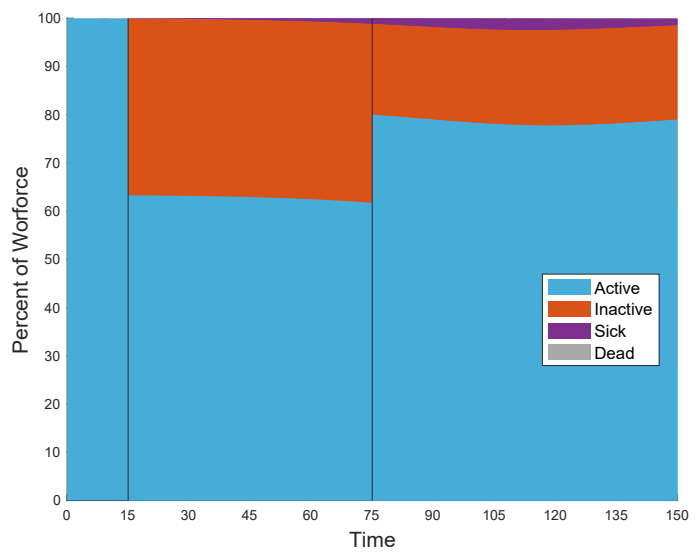

(c) Sacramento, Healthcare Outcomes

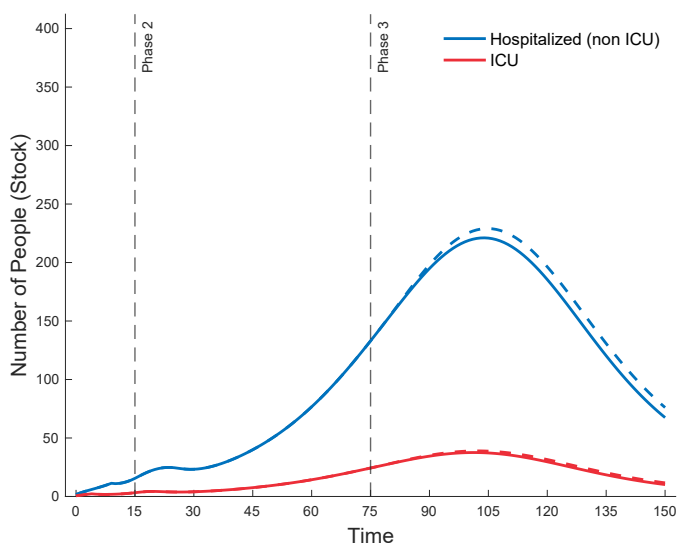

Note: The figure shows health and employment outcomes with No Policy in Phase 1, Essential Only in Phase 2 and Alternating Schedules in Phase 3 for the Sacramento MSA, with contact matrices based on Replica data. The top left panel displays the percent of individuals of the local population that are infected and symptomatic and/or detected, and the number of deaths per 100,000s of population on the right vertical axis. The top right panel corresponds plots the share of workforce that is either active, inactive (due to quarantine, or not allowed to access the workplace and unable to work from home), sick, or deceased. Dashed lines correspond to the baseline case NP-EO-CR. 
Figure C.15: NP-CR-CR Policy Sequence in Sacramento (Replica Based)

(a) Sacramento, Epidemic Outcomes

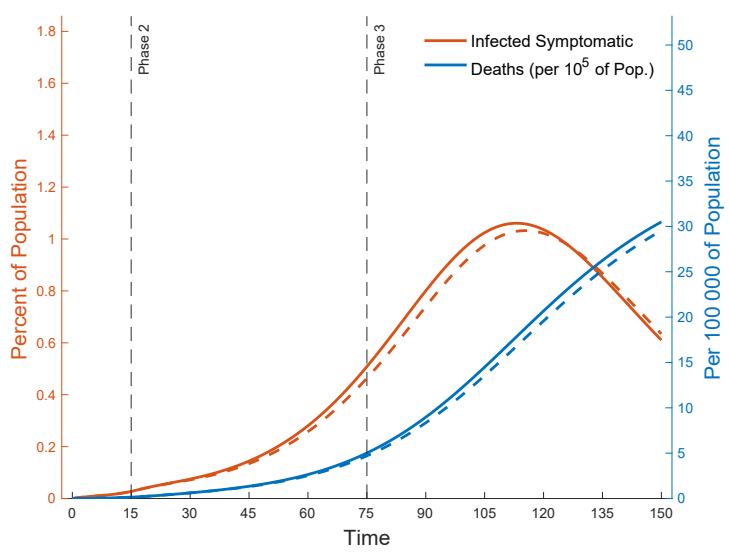

(b) Sacramento, Employment Outcomes

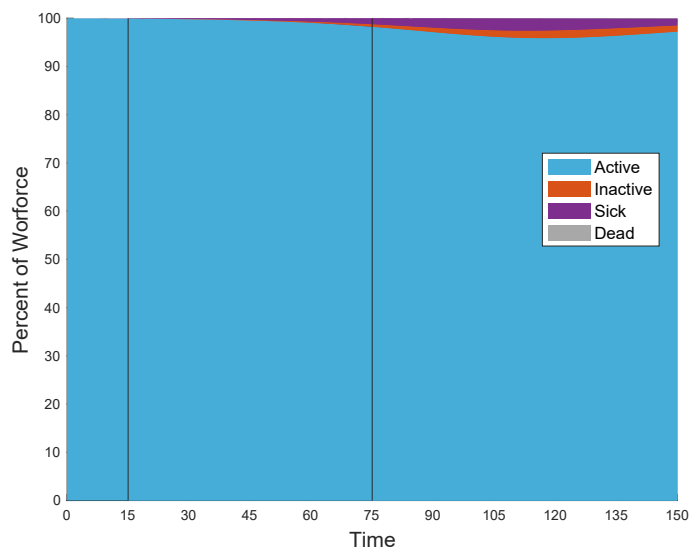

(c) Sacramento, Healthcare Outcomes

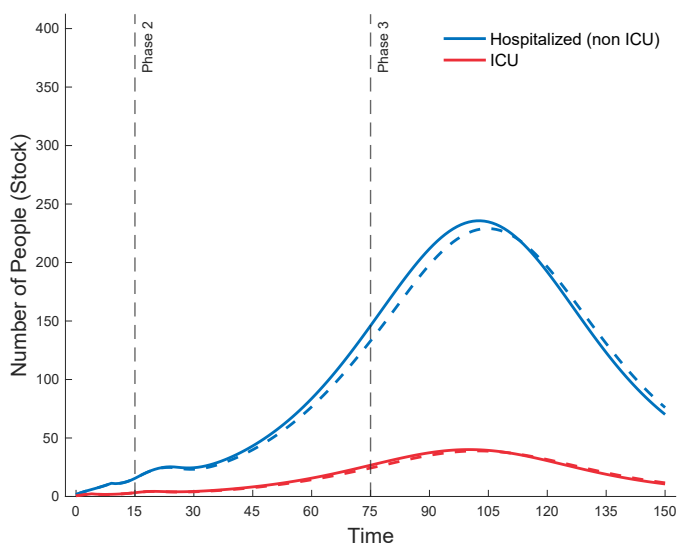

Note: The figure shows health and employment outcomes with No Policy in Phase 1, Cautious Reopening in Phase 2 and Cautious Reopening in Phase 3 for the Sacramento MSA, with contact matrices based on Replica data. The top left panel displays the percent of individuals of the local population that are infected and symptomatic and/or detected, and the number of deaths per 100,000s of population on the right vertical axis. The top right panel corresponds plots the share of workforce that is either active, inactive (due to quarantine, or not allowed to access the workplace and unable to work from home), sick, or deceased. Dashed lines correspond to the baseline case NP-EO-CR. 
Figure C.16: NP-60+-60+ Policy Sequence in Sacramento (Replica Based)

(a) Sacramento, Epidemic Outcomes

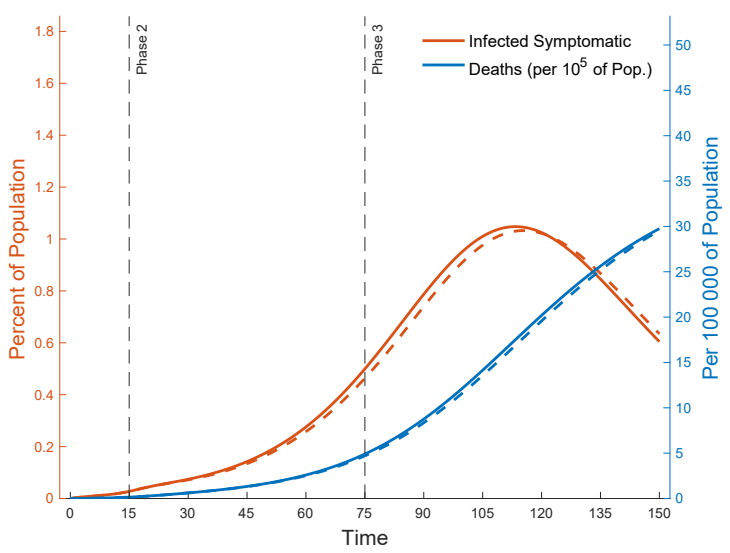

(b) Sacramento, Employment Outcomes

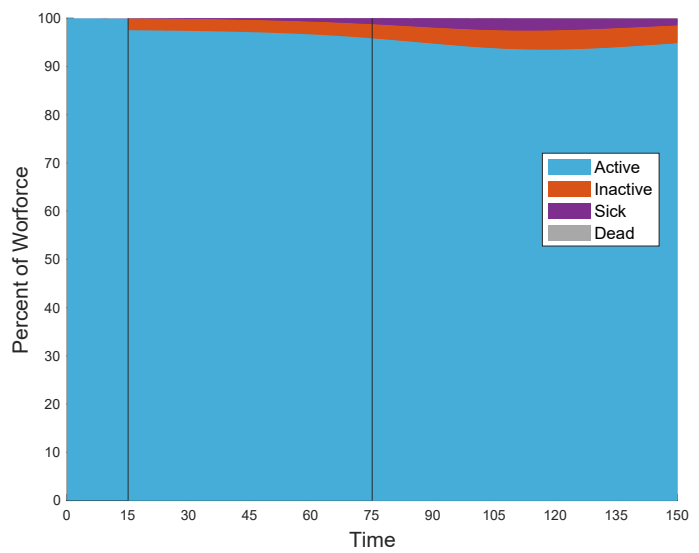

(c) Sacramento, Healthcare Outcomes

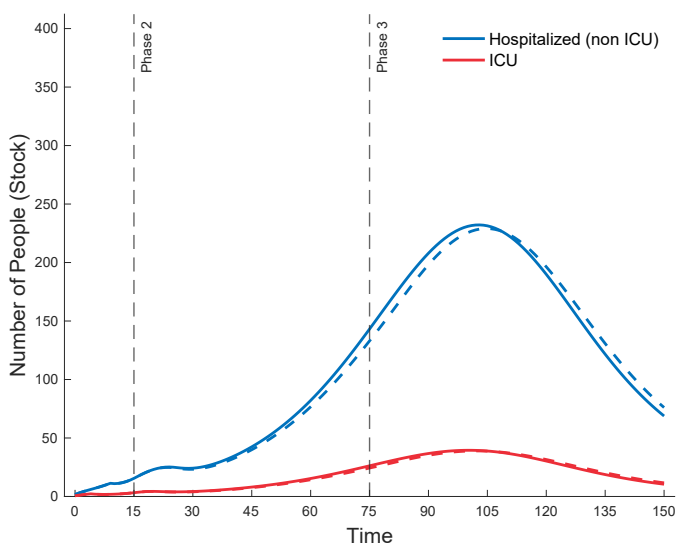

Note: The figure shows health and employment outcomes with No Policy in Phase 1, Isolate 60+ in Phase 2 and Isolate $60+$ in Phase 3 for the Sacramento MSA, with contact matrices based on Replica data. The top left panel displays the percent of individuals of the local population that are infected and symptomatic and/or detected, and the number of deaths per 100,000s of population on the right vertical axis. The top right panel corresponds plots the share of workforce that is either active, inactive (due to quarantine, or not allowed to access the workplace and unable to work from home), sick, or deceased. Dashed lines correspond to the baseline case NP-EO-CR. 


\section{Figure C.17: NP-WFH-WFH Policy Sequence in Sacramento (Replica Based)}

(a) Sacramento, Epidemic Outcomes

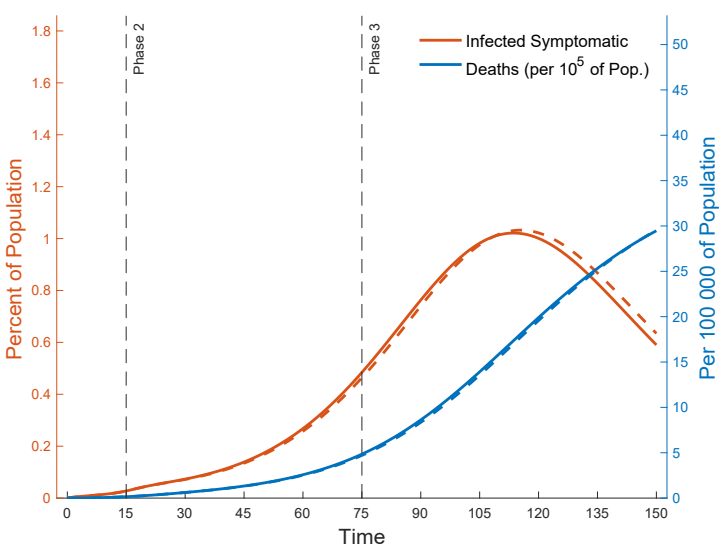

(b) Sacramento, Employment Outcomes

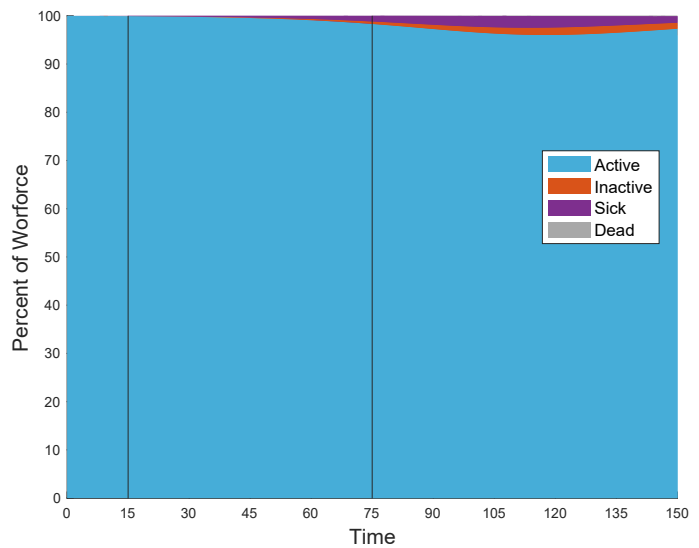

(c) Sacramento, Healthcare Outcomes

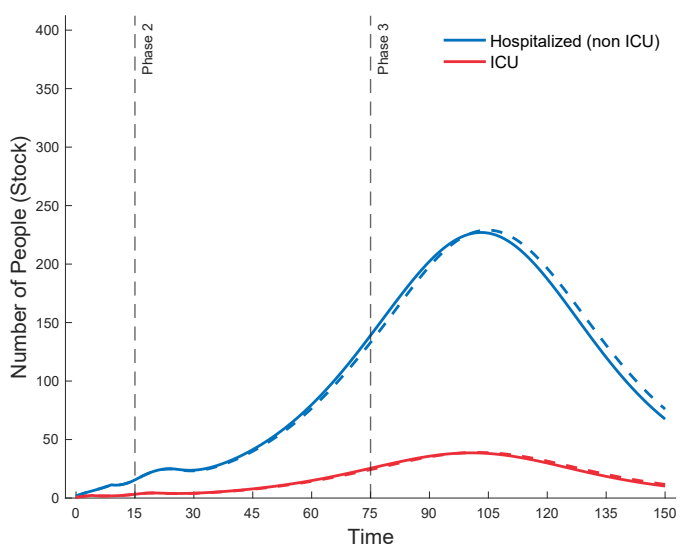

Note: The figure shows health and employment outcomes with No Policy in Phase 1, Work-from-Home if Possible in Phase 2 and Work-from-Home if Possible in Phase 3 for the Sacramento MSA, with contact matrices based on Replica data. The top left panel displays the percent of individuals of the local population that are infected and symptomatic and/or detected, and the number of deaths per 100,000s of population on the right vertical axis. The top right panel corresponds plots the share of workforce that is either active, inactive (due to quarantine, or not allowed to access the workplace and unable to work from home), sick, or deceased. Dashed lines correspond to the baseline case NP-EO-CR. 
Figure C.18: NP-AS-AS Policy Sequence in Sacramento (Replica Based)

(a) Sacramento, Epidemic Outcomes

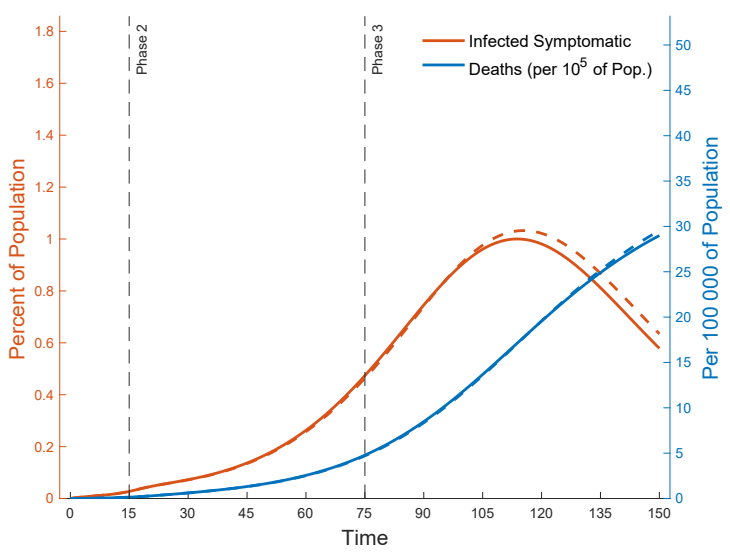

(b) Sacramento, Employment Outcomes

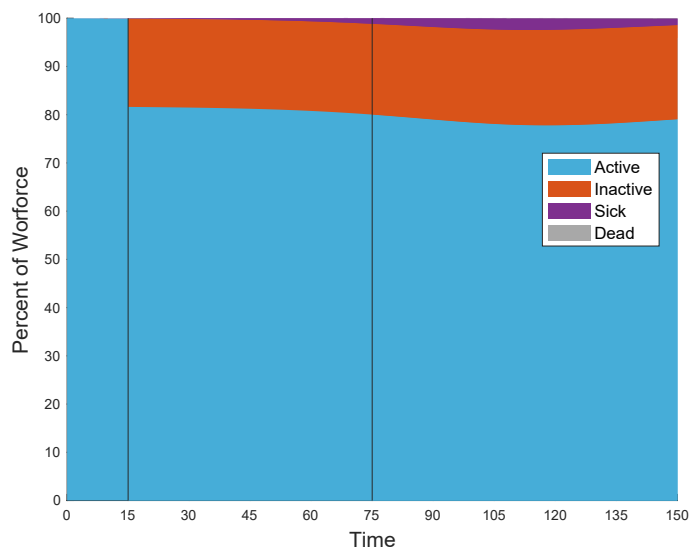

(c) Sacramento, Healthcare Outcomes

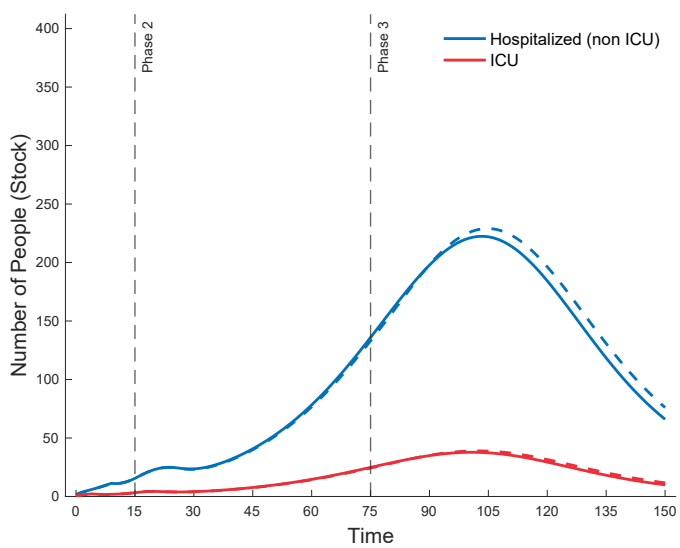

Note: The figure shows health and employment outcomes with No Policy in Phase 1, Alternating Schedules in Phase 2 and Alternating Schedules in Phase 3 for the Sacramento MSA, with contact matrices based on Replica data. The top left panel displays the percent of individuals of the local population that are infected and symptomatic and/or detected, and the number of deaths per 100,000s of population on the right vertical axis. The top right panel corresponds plots the share of workforce that is either active, inactive (due to quarantine, or not allowed to access the workplace and unable to work from home), sick, or deceased. Dashed lines correspond to the baseline case NP-EO-CR. 
Figure C.19: NP-EO-CR Policy Sequence in New York (FRED Based)

(a) New York, Epidemic Outcomes

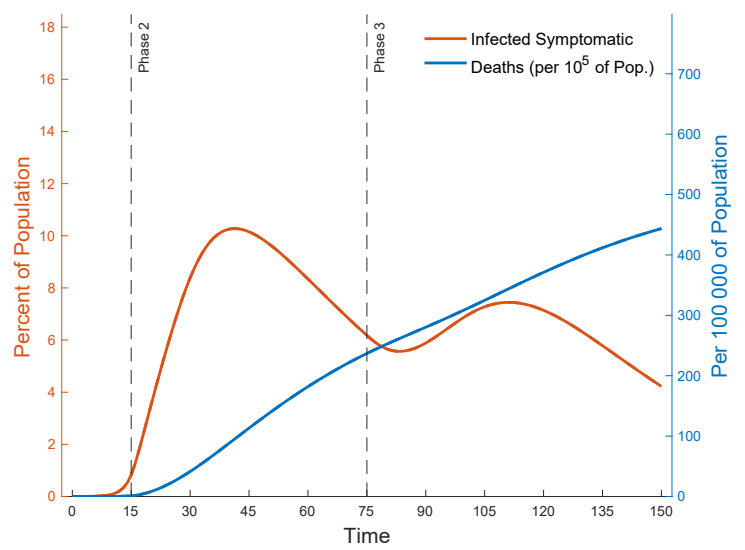

(b) New York, Employment Outcomes

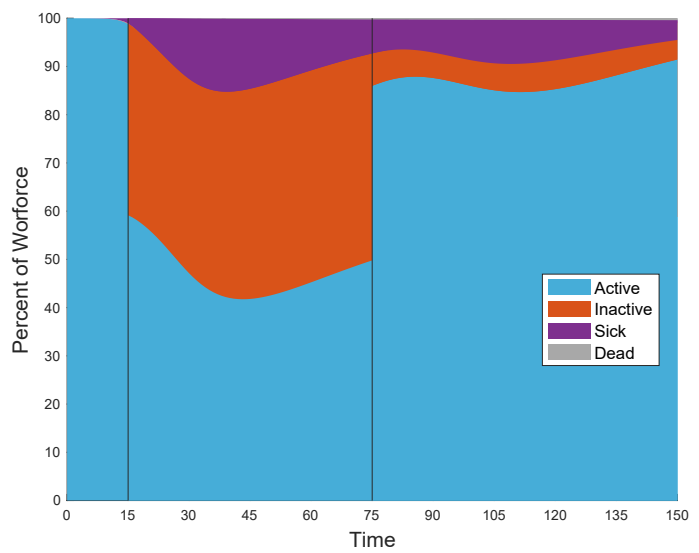

(c) New York, Healthcare Outcomes

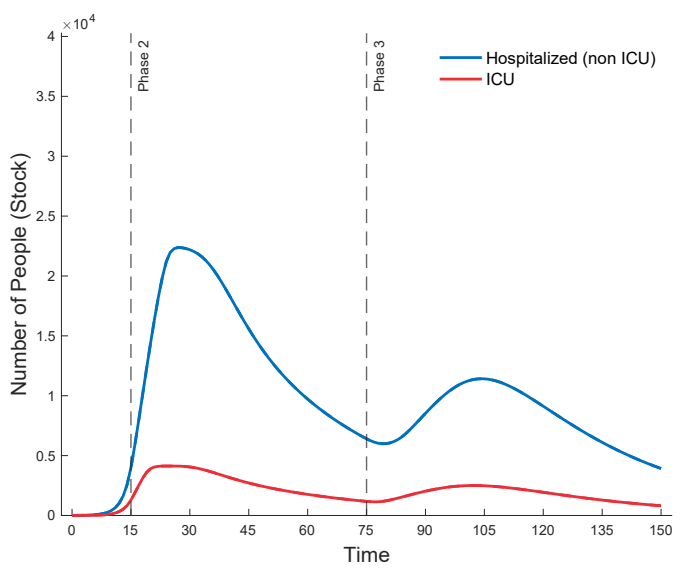

Note: The figure shows health and employment outcomes with No Policy in Phase 1, Essential Only in Phase 2 and Cautious Reopening in Phase 3 for the New York MSA, with contact matrices based on FRED data. The top left panel displays the percent of individuals of the local population that are infected and symptomatic and/or detected, and the number of deaths per 100,000s of population on the right vertical axis. The top right panel corresponds plots the share of workforce that is either active, inactive (due to quarantine, or not allowed to access the workplace and unable to work from home), sick, or deceased. Dashed lines correspond to the baseline case NP-EO-CR. 
Figure C.20: NP-EO-EO Policy Sequence in New York (FRED Based)

(a) New York, Epidemic Outcomes

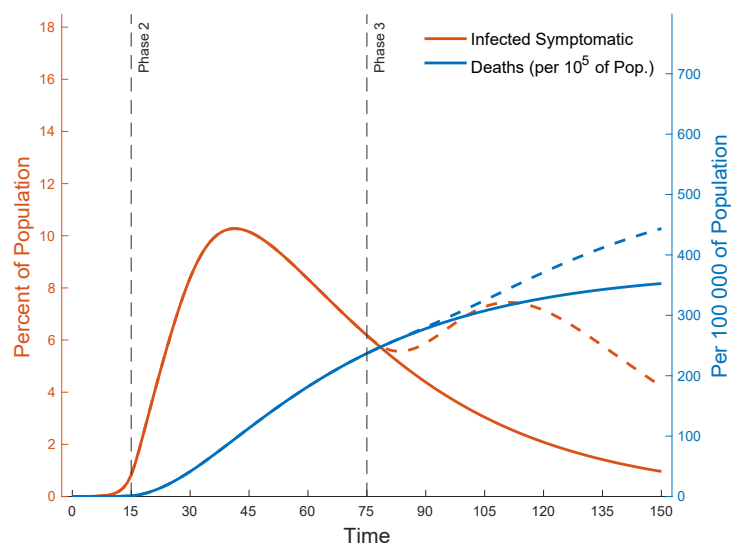

(b) New York, Employment Outcomes

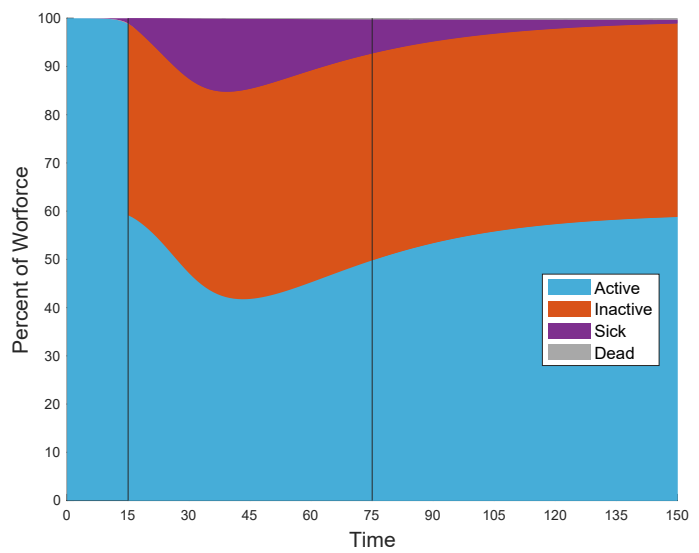

(c) New York, Healthcare Outcomes

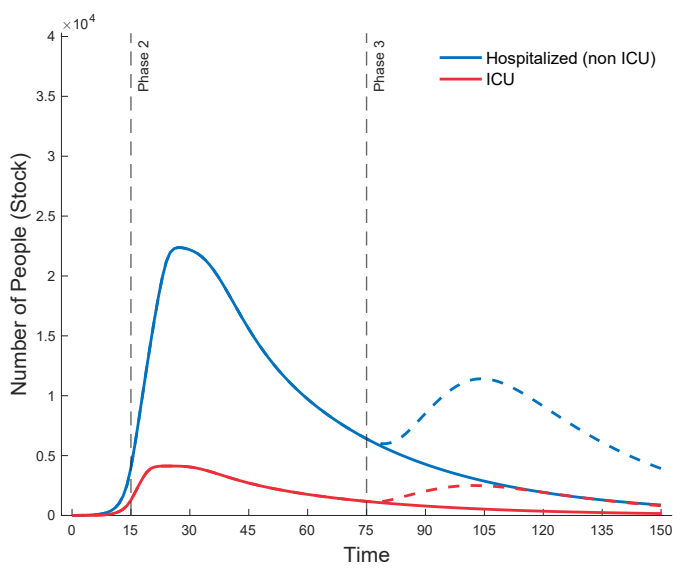

Note: The figure shows health and employment outcomes with No Policy in Phase 1, Essential Only in Phase 2 and Essential Only in Phase 3 for the New York MSA, with contact matrices based on FRED data. The top left panel displays the percent of individuals of the local population that are infected and symptomatic and/or detected, and the number of deaths per 100,000s of population on the right vertical axis. The top right panel corresponds plots the share of workforce that is either active, inactive (due to quarantine, or not allowed to access the workplace and unable to work from home), sick, or deceased. Dashed lines correspond to the baseline case NP-EO-CR. 
Figure C.21: NP-EO-60+ Policy Sequence in New York (FRED Based)

(a) New York, Epidemic Outcomes

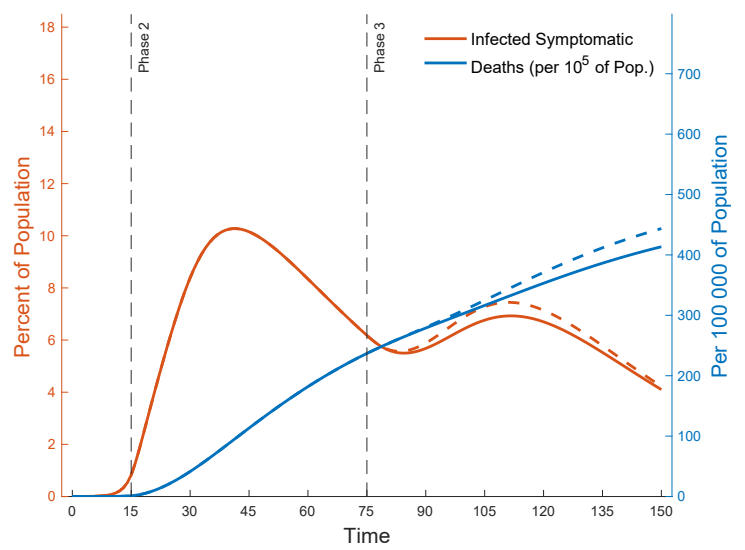

(b) New York, Employment Outcomes

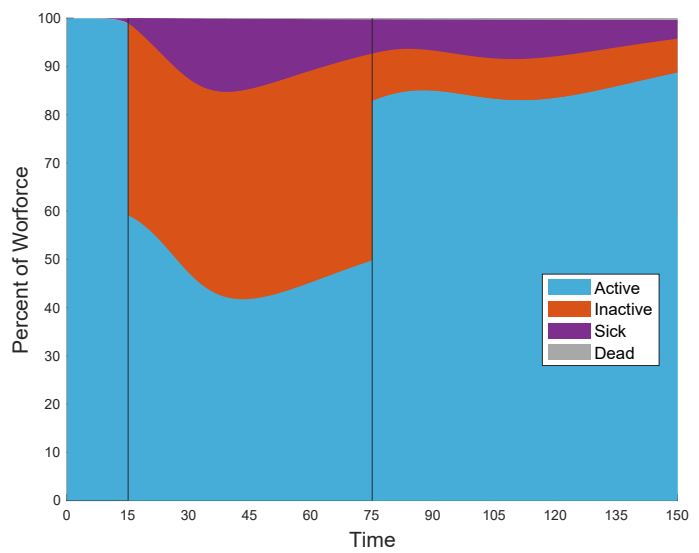

(c) New York, Healthcare Outcomes

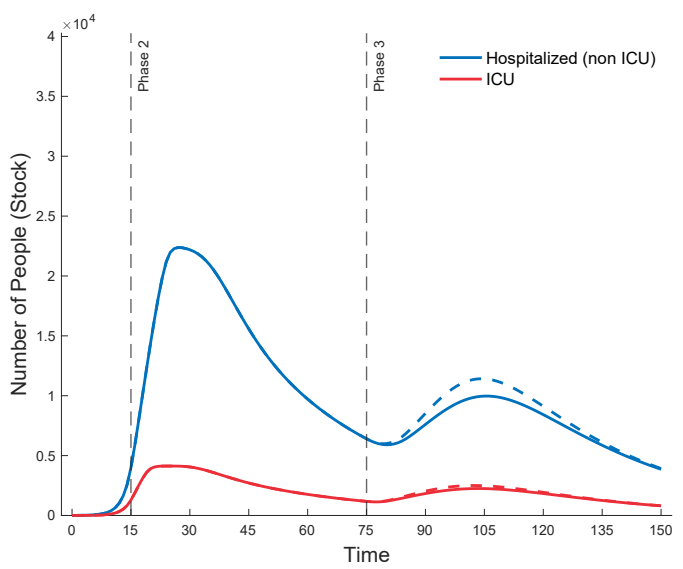

Note: The figure shows health and employment outcomes with No Policy in Phase 1, Essential Only in Phase 2 and Isolate 60+ in Phase 3 for the New York MSA, with contact matrices based on FRED data. The top left panel displays the percent of individuals of the local population that are infected and symptomatic and/or detected, and the number of deaths per 100,000s of population on the right vertical axis. The top right panel corresponds plots the share of workforce that is either active, inactive (due to quarantine, or not allowed to access the workplace and unable to work from home), sick, or deceased. Dashed lines correspond to the baseline case NP-EO-CR. 
Figure C.22: NP-EO-WFH Policy Sequence in New York (FRED Based)

(a) New York, Epidemic Outcomes

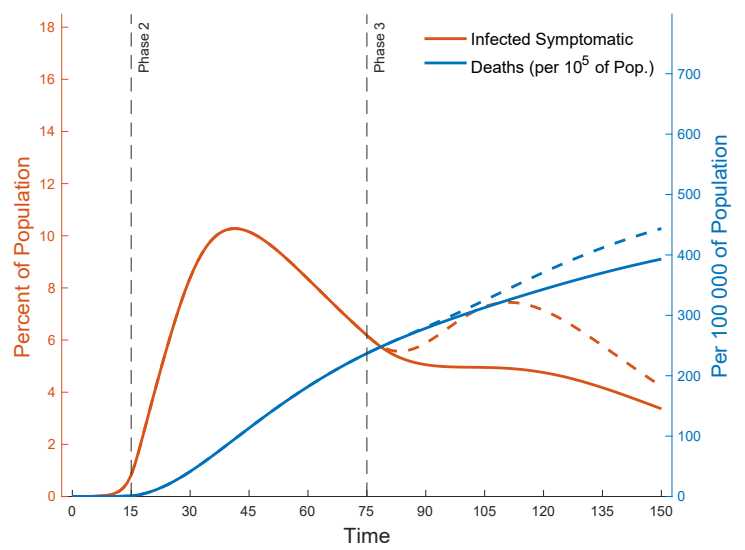

(b) New York, Employment Outcomes

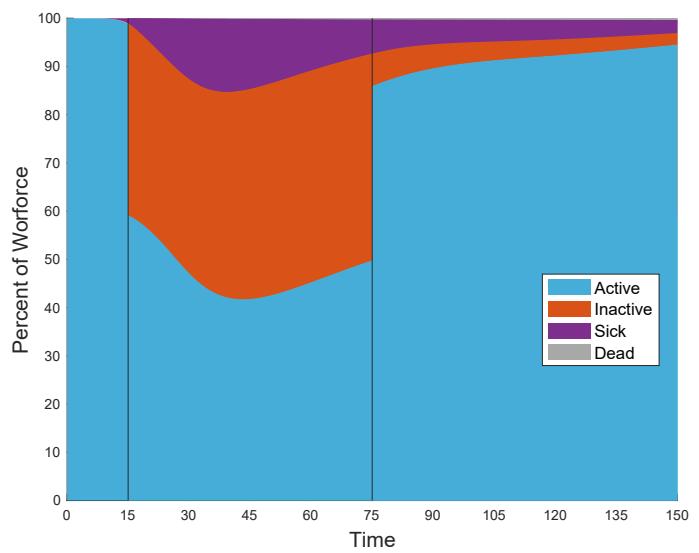

(c) New York, Healthcare Outcomes

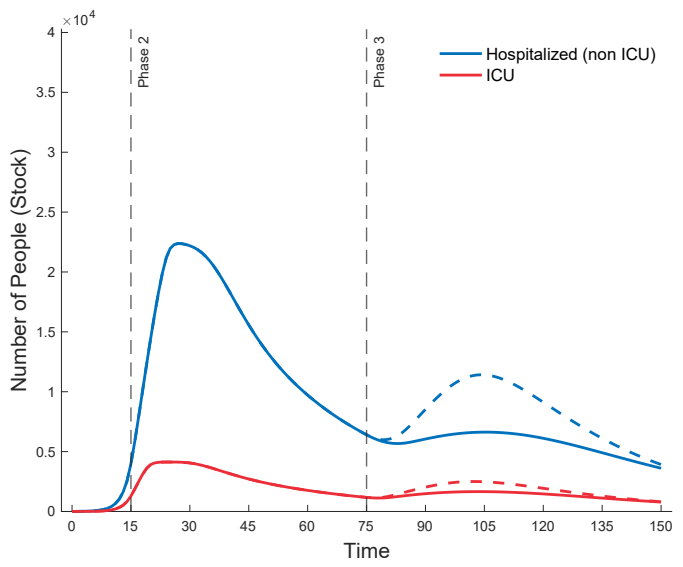

Note: The figure shows health and employment outcomes with No Policy in Phase 1, Essential Only in Phase 2 and Work-from-Home if Possible in Phase 3 for the New York MSA, with contact matrices based on FRED data. The top left panel displays the percent of individuals of the local population that are infected and symptomatic and/or detected, and the number of deaths per 100,000s of population on the right vertical axis. The top right panel corresponds plots the share of workforce that is either active, inactive (due to quarantine, or not allowed to access the workplace and unable to work from home), sick, or deceased. Dashed lines correspond to the baseline case NP-EO-CR. 
Figure C.23: NP-EO-AS Policy Sequence in New York (FRED Based)

(a) New York, Epidemic Outcomes

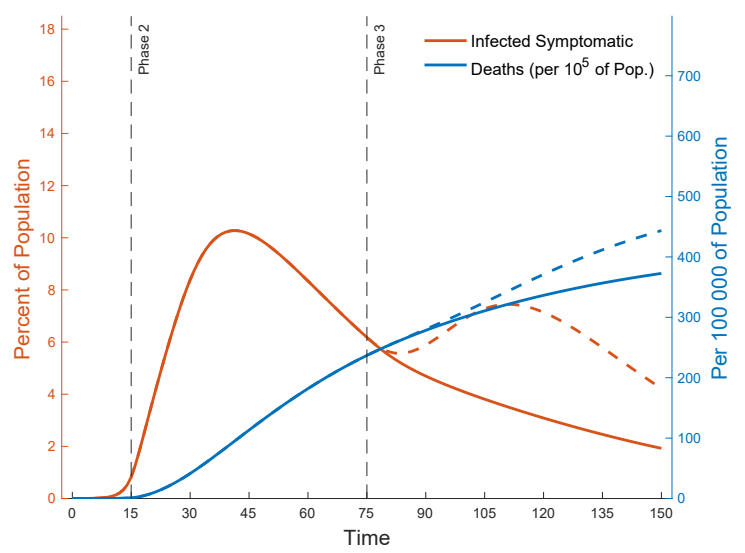

(b) New York, Employment Outcomes

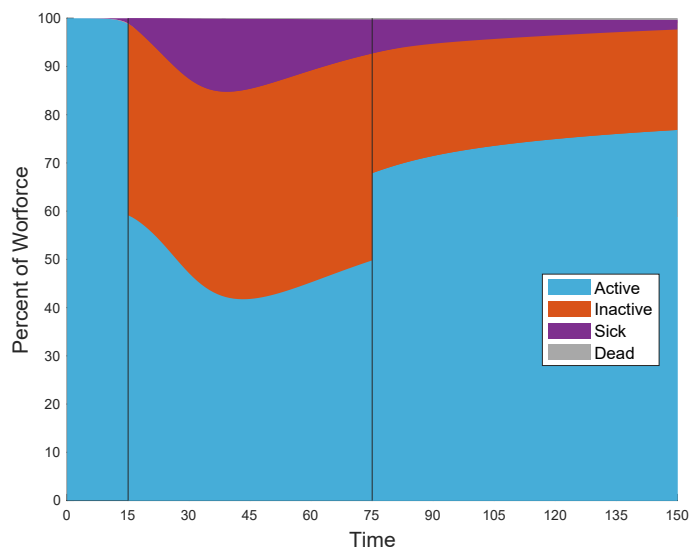

(c) New York, Healthcare Outcomes

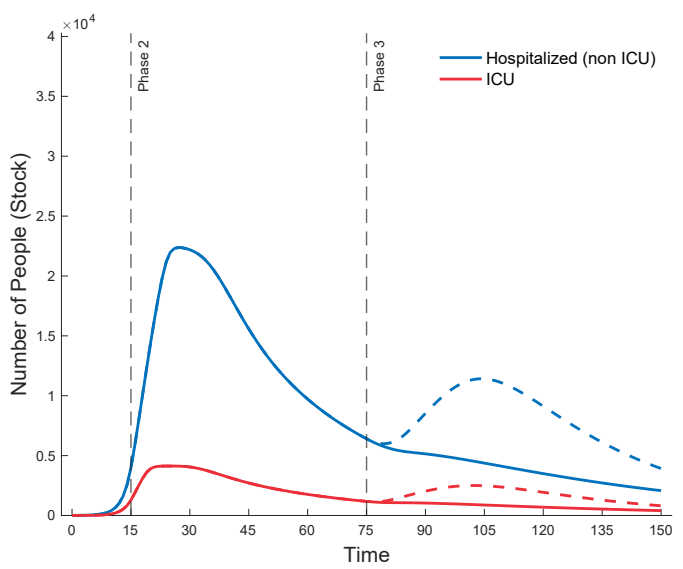

Note: The figure shows health and employment outcomes with No Policy in Phase 1, Essential Only in Phase 2 and Alternating Schedules in Phase 3 for the New York MSA, with contact matrices based on FRED data. The top left panel displays the percent of individuals of the local population that are infected and symptomatic and/or detected, and the number of deaths per 100,000s of population on the right vertical axis. The top right panel corresponds plots the share of workforce that is either active, inactive (due to quarantine, or not allowed to access the workplace and unable to work from home), sick, or deceased. Dashed lines correspond to the baseline case NP-EO-CR. 
Figure C.24: NP-CR-CR Policy Sequence in New York (FRED Based)

(a) New York, Epidemic Outcomes

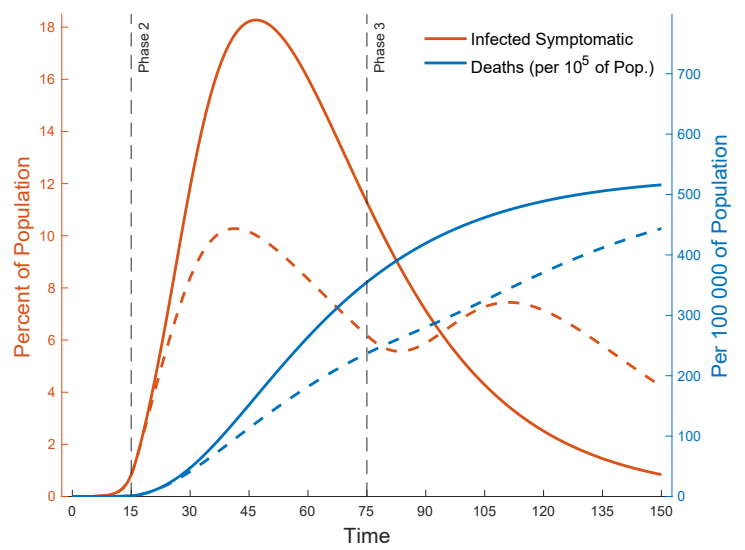

(b) New York, Employment Outcomes

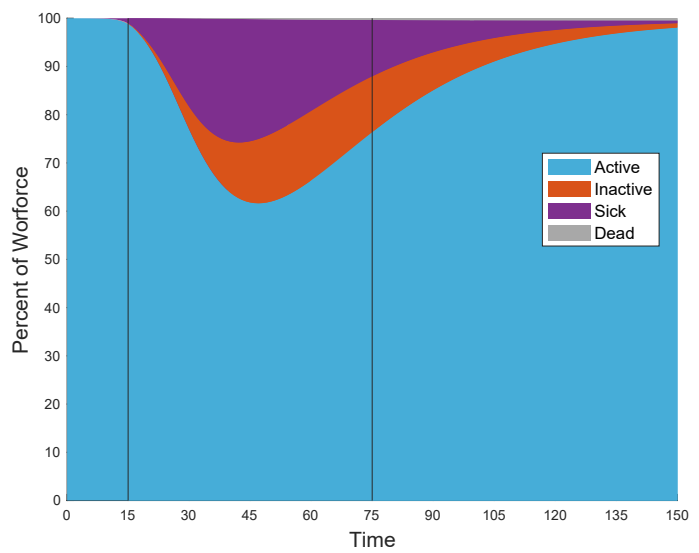

(c) New York, Healthcare Outcomes

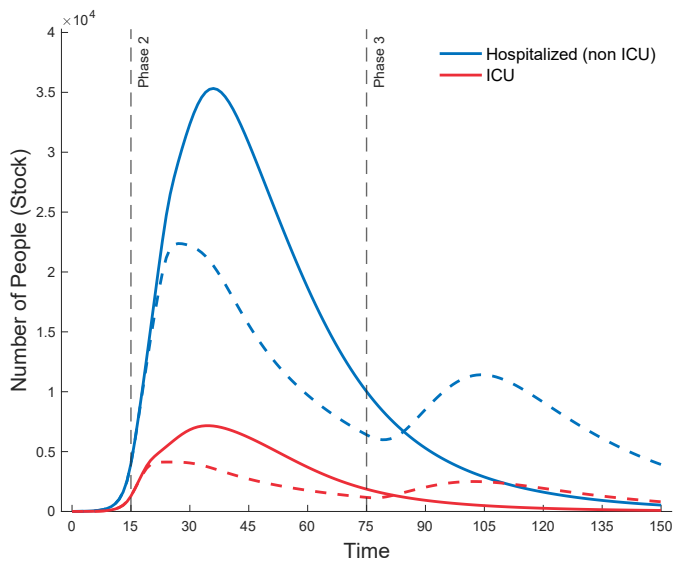

Note: The figure shows health and employment outcomes with No Policy in Phase 1, Cautious Reopening in Phase 2 and Cautious Reopening in Phase 3 for the New York MSA, with contact matrices based on FRED data. The top left panel displays the percent of individuals of the local population that are infected and symptomatic and/or detected, and the number of deaths per 100,000s of population on the right vertical axis. The top right panel corresponds plots the share of workforce that is either active, inactive (due to quarantine, or not allowed to access the workplace and unable to work from home), sick, or deceased. Dashed lines correspond to the baseline case NP-EO-CR. 
Figure C.25: NP-60+-60+ Policy Sequence in New York (FRED Based)

(a) New York, Epidemic Outcomes

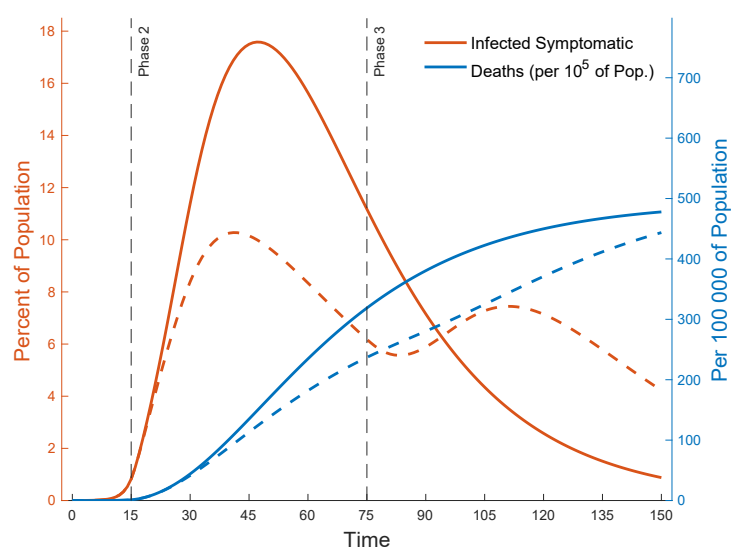

(b) New York, Employment Outcomes

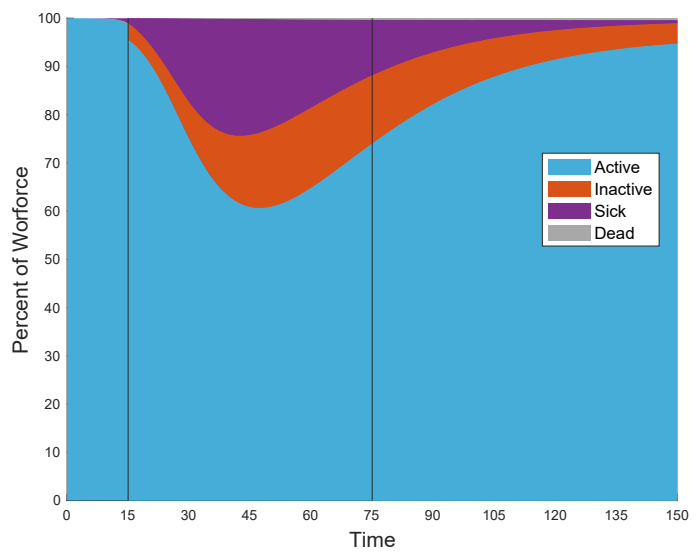

(c) New York, Healthcare Outcomes

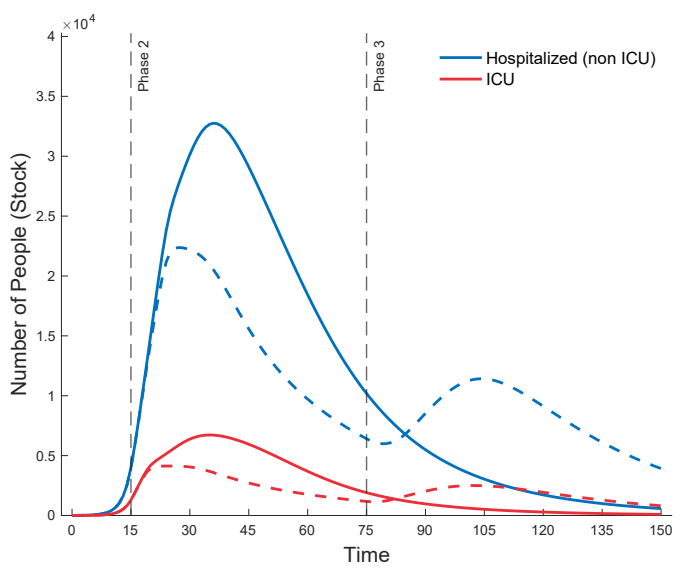

Note: The figure shows health and employment outcomes with No Policy in Phase 1, Isolate 60+ in Phase 2 and Isolate 60+ in Phase 3 for the New York MSA, with contact matrices based on FRED data. The top left panel displays the percent of individuals of the local population that are infected and symptomatic and/or detected, and the number of deaths per 100,000s of population on the right vertical axis. The top right panel corresponds plots the share of workforce that is either active, inactive (due to quarantine, or not allowed to access the workplace and unable to work from home), sick, or deceased. Dashed lines correspond to the baseline case NP-EO-CR. 
Figure C.26: NP-WFH-WFH Policy Sequence in New York (FRED Based)

(a) New York, Epidemic Outcomes

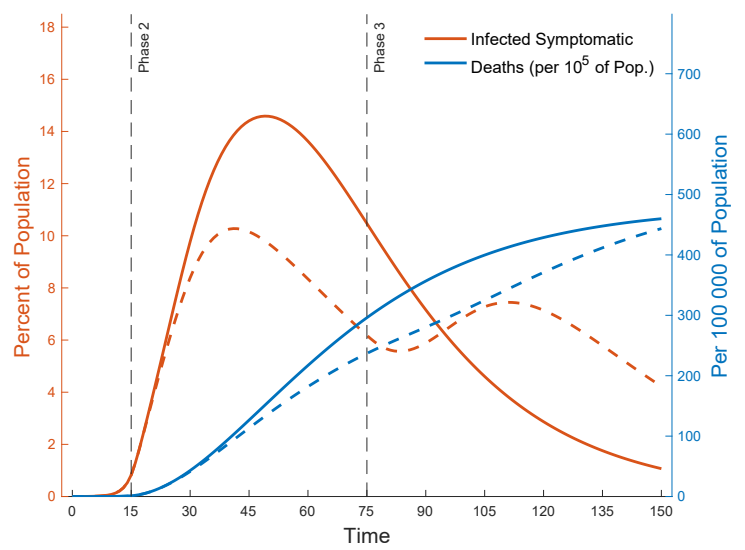

(b) New York, Employment Outcomes

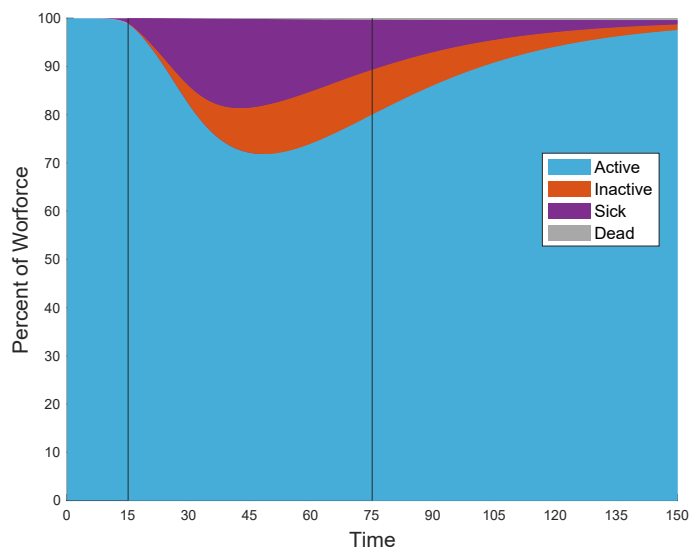

(c) New York, Healthcare Outcomes

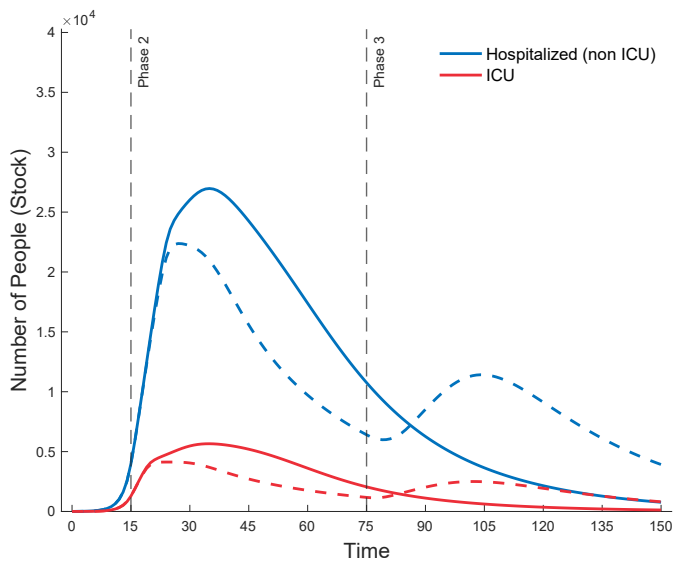

Note: The figure shows health and employment outcomes with No Policy in Phase 1, Work-from-Home if Possible in Phase 2 and Work-from-Home if Possible in Phase 3 for the New York MSA, with contact matrices based on FRED data. The top left panel displays the percent of individuals of the local population that are infected and symptomatic and/or detected, and the number of deaths per 100,000s of population on the right vertical axis. The top right panel corresponds plots the share of workforce that is either active, inactive (due to quarantine, or not allowed to access the workplace and unable to work from home), sick, or deceased. Dashed lines correspond to the baseline case NP-EO-CR. 
Figure C.27: NP-AS-AS Policy Sequence in New York (FRED Based)

(a) New York, Epidemic Outcomes

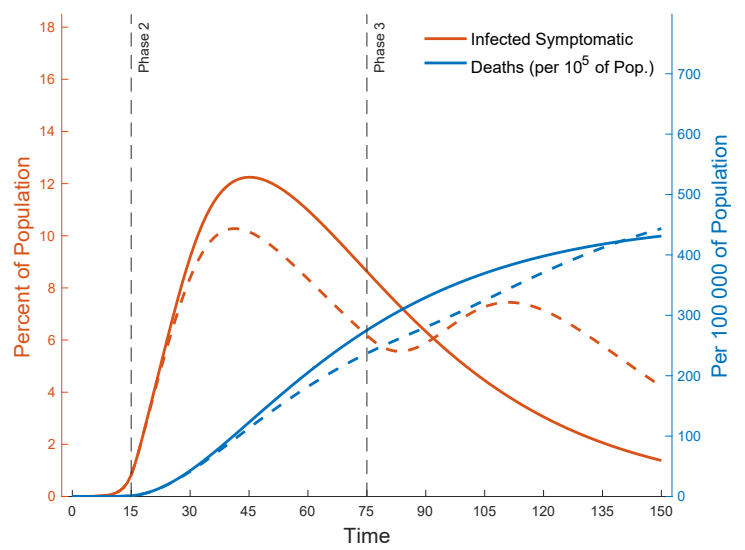

(b) New York, Employment Outcomes

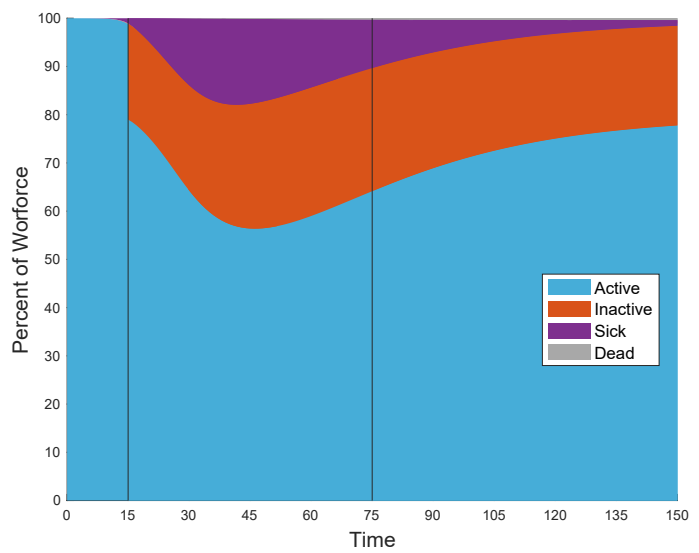

(c) New York, Healthcare Outcomes

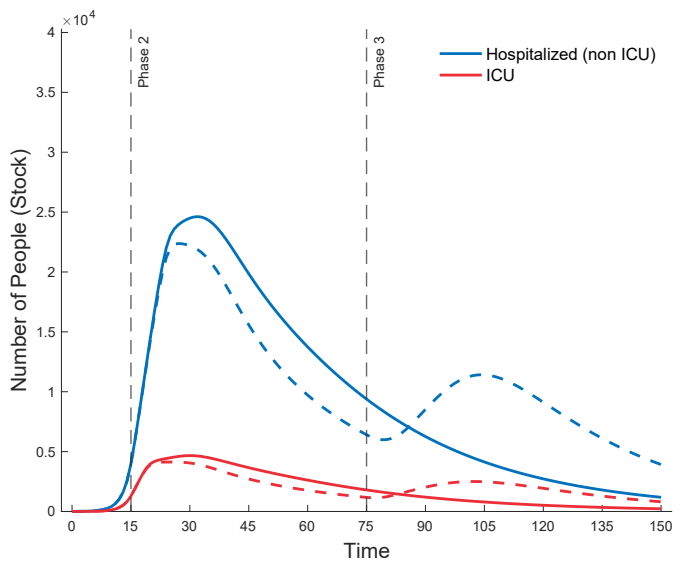

Note: The figure shows health and employment outcomes with No Policy in Phase 1, Alternating Schedules in Phase 2 and Alternating Schedules in Phase 3 for the New York MSA, with contact matrices based on FRED data. The top left panel displays the percent of individuals of the local population that are infected and symptomatic and/or detected, and the number of deaths per 100,000s of population on the right vertical axis. The top right panel corresponds plots the share of workforce that is either active, inactive (due to quarantine, or not allowed to access the workplace and unable to work from home), sick, or deceased. Dashed lines correspond to the baseline case NP-EO-CR. 